\title{
CONVERGENCE AND STOCHASTIC HOMOGENIZATION OF A CLASS OF TWO COMPONENTS NONLINEAR REACTION-DIFFUSION SYSTEMS
}

\author{
OMAR ANZA HAFSA, JEAN PHILIPPE MANDALLENA AND GÉRARD MICHAILLE
}

\begin{abstract}
We establish a convergence theorem for a class of two components nonlinear reactiondiffusion systems. Each diffusion term is the subdifferential of a convex functional of the calculus of variations whose class is equipped with the Mosco-convergence. The reaction terms are structured in such a way that the systems admit bounded solutions, which are positive in the modeling of ecosystems. As a consequence, under a stochastic homogenization framework, we prove two homogenization theorems for this class. We illustrate the results with the stochastic homogenization of a prey-predator model with saturation effect.
\end{abstract}

\section{Contents}

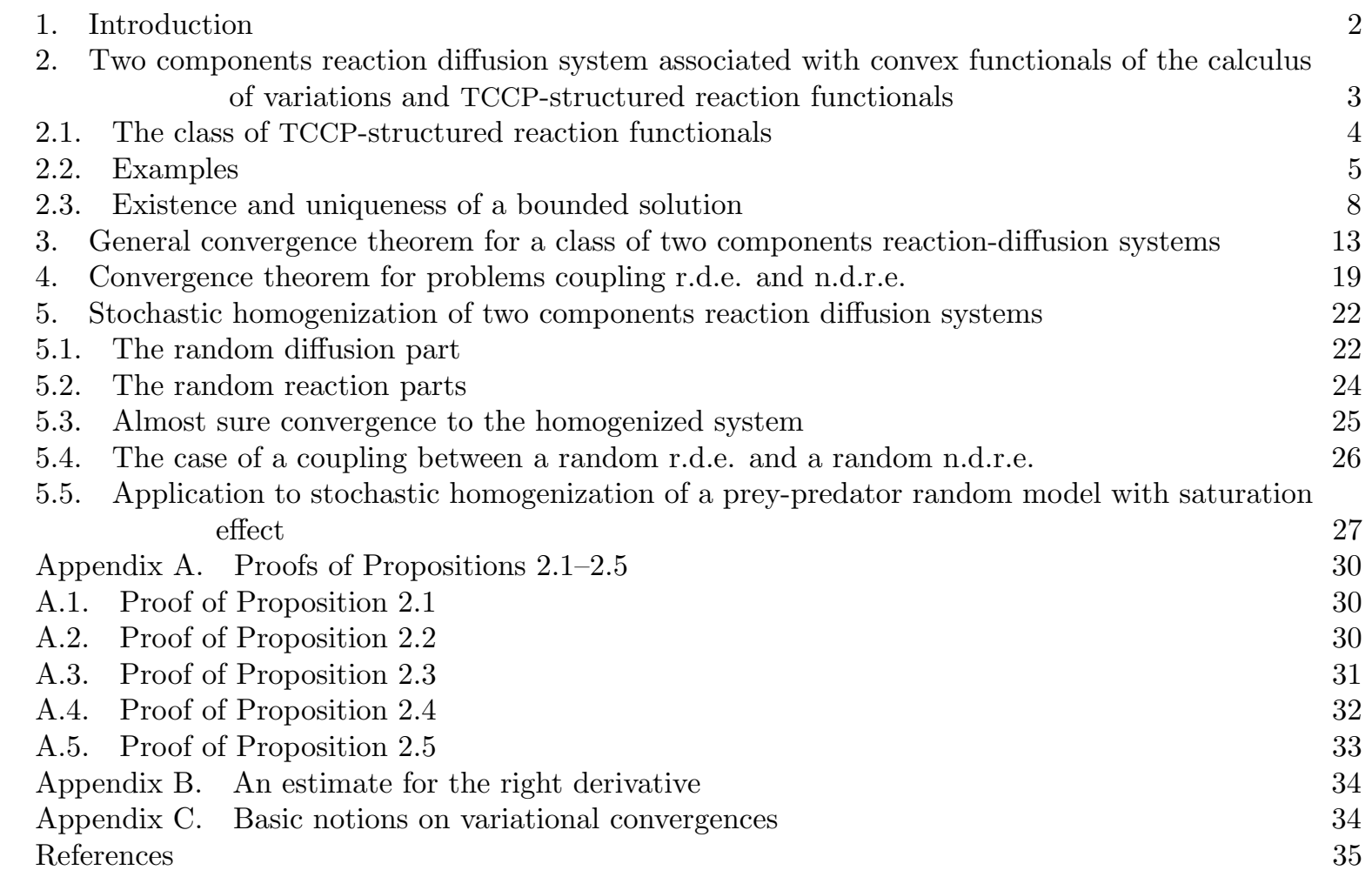

Universite de nimes, laboratoire MiPA, Site des Carmes, Place Gabriel Péri, 30021 Nîmes, France. E-mail address: (Omar Anza Hafsa) omar.anza-hafsa@unimes.fr, (Jean Philippe Mandallena) jean-philippe.mandallena@unimes.fr, (Gérard Michaille) gerard.michaille@gmail.com.

2010 Mathematics Subject Classification. 35K57, 35B27, 49J45.

Key words and phrases. Convergence of two components reaction-diffusion equations, stochastic homogenization, preypredator models. 


\section{INTRODUCTION}

Let $T>0$ and $\Omega$ be a bounded domain in $\mathbb{R}^{N}$. The paper is concerned with the convergence of sequences of reaction-diffusion systems in $L^{2}\left(0, T, L^{2}(\Omega)\right)$ of the type

$$
\left(\mathcal{S}_{n}\right)\left\{\begin{array}{l}
\frac{d u_{n}}{d t}(t)+\partial \Phi_{1, n}\left(u_{n}(t)\right) \ni F_{1, n}\left(t, u_{n}(t), v_{n}(t)\right) \text { for a.e. } t \in(0, T) \\
\frac{d v_{n}}{d t}(t)+\partial \Phi_{2, n}\left(v_{n}(t)\right) \ni F_{2, n}\left(t, u_{n}(t), v_{n}(t)\right) \text { for a.e. } t \in(0, T) \\
\underline{\rho}_{1, n} \leq u_{n}(0) \leq \bar{\rho}_{1, n}, \underline{\rho}_{2, n} \leq v_{n}(0) \leq \bar{\rho}_{2, n}, u_{n}(0) \in \overline{\operatorname{dom}\left(\partial \Phi_{1, n}\right)}, v_{n}(0) \in \overline{\operatorname{dom}\left(\partial \Phi_{2, n}\right)}
\end{array}\right.
$$

where, for $i=1,2, \underline{\rho}_{i, n}$ and $\bar{\rho}_{i, n}$ are suitable constants depending on the reaction functional $F_{i, n}$. Each diffusion term is the subdifferential of a convex integral functional of the calculus of variations $\Phi_{i, n}: L^{2}(\Omega) \rightarrow \mathbb{R} \cup\{+\infty\}$, whose domain dom $\left(\partial \Phi_{i, n}\right)$ contains the boundary conditions. In their domain, the diffusion terms are of divergence form $-\operatorname{div} D_{\xi} W_{1, n}(x, \nabla u)$ and $-\operatorname{div} D_{\xi} W_{2, n}(x, \nabla v)$. The explicit dependence on the spatial variable reflects the fact that the diffusion may take place in heterogeneous media. More specifically, when we write $n$ for $\varepsilon_{n}$ intended to tend toward 0 , the system models among other examples, ecosystems of two species in a spatial domain made up of small habitats with size $\varepsilon_{n}$. The well-posedness nature of reaction-diffusion systems in the sense of existence of a strong solution has been extensively studied. In this paper, the novelty is the special form of the reactions functionals: they are structured in such a way that for fixed $v \in L^{2}(\Omega),(t, u) \mapsto F_{n, 1}(t, u, v)$, and for fixed $u \in L^{2}(\Omega)$, $(t, v) \mapsto F_{n, 2}(t, u, v)$ are SVR-structured reaction functionals as defined in [1]. As a consequence, $\left(\mathcal{S}_{n}\right)$ admits a pair of bounded solutions according to the constants $\underline{\rho}_{i, n}$ and $\bar{\rho}_{i, n}$ that govern the initial conditions. Problems $\left(\mathcal{S}_{n}\right)$ model various situations involving competition or symbiosis models, prey predator models in ecology, as well as heat mass transfert in chemical reactors and combustion theory, or gaz-liquid interactions problems, etc. They are illustrated through Examples 2.1, 2.2, 2.3, 2.4. It should be noted that our study includes systems $\left(S_{n}\right)$ coupling a reaction-diffusion equation (r.d.e.) and a non diffusive reaction equation (n.d.r.e.), like the FitzHugh-Nagumo system in neurophysiology described in Example 2.5.

In Section 2, for any $T>0$, we prove existence and uniqueness of bounded strong solutions in $C\left([0, T], L^{2}(\Omega)\right) \times C\left([0, T], L^{2}(\Omega)\right)$ for problems of the type $\left(\mathcal{S}_{n}\right)$, when initial functions are bounded according to the reaction functionals. The proof is based on [1, Theorem 3.1] combined with a suitable fixed point procedure.

In Section 3, under the Mosco-convergence of functionals $\Phi_{i, n}$, and a suitable convergence of $F_{i, n}$ to $F_{i}, i=1,2$, we establish the first main result of the paper, Theorem 3.1. which states the convergence of $\left(\mathcal{S}_{n}\right)$ toward a reaction-diffusion system $(\mathcal{S})$ of the same type. This can be seen as a compactness or a stability result for the class of systems considered.

The convergence of systems $\left(\mathcal{S}_{n}\right)$ coupling a reaction-diffusion equation with a non diffusive reaction equation or two non diffusive reaction equations is addressed in Section 4 and is discussed in Theorem 4.1 and Theorem 4.2. It requires additional regularity conditions on the reaction functional associated with the n.d.r.e..

As far as we know, the homogenization of reaction-diffusion systems was first addressed in [10, 14, by means of the two scale convergence; see also 13 where the homogenization with evolving microstructure is performed using the method of transformation to a periodic reference domain. In Section 5 we hope to contribute to this research in the framework of stochastic homogenization described in [1]. The main results, which are direct consequences of Theorems 3.1, 4.1, are stated in Theorems 5.1, 5.2, They are illustrated through the homogenization of a prey-predator model with a saturation effect which is the randomization of Example 2.3. The model involves two species spreading in an heterogeneous environment whose small spatial heterogeneities are randomly distributed following a Poisson point process. The homogenized problem illustrates the interplay between the growth rate of the prey and the maximum carrying capacity of the environment when the size of the spatial heterogeneities is very small. 


\section{TWO COMPONENTS REACTION DIFFUSION SYSTEM ASSOCIATED WITH CONVEX FUNCTIONALS OF THE CALCULUS OF VARIATIONS AND TCCP-STRUCTURED REACTION FUNCTIONALS}

We denote by $\mathcal{L}_{N}$ the Lebesgue measure in $\mathbb{R}^{N}$, by $\Omega$ a domain of $\mathbb{R}^{N}$ of class $C^{1}$, and by $\Gamma$ a subset of its boundary $\partial \Omega$ with positive $\mathcal{H}^{N-1}$-Hausdorf measure. To shorten the notation, we sometimes write $X$ to denote the Hilbert space $L^{2}(\Omega)$ equipped with its standard scalar product and its associated norm, denoted by $\langle$,$\rangle and \|.\|_{X}$ respectively. All along the paper we use the same notation $|\cdot|$ to denote the norms of the euclidean spaces $\mathbb{R}^{d}, d \geq 1$, and by $\xi \cdot \xi^{\prime}$ the standard scalar product of two elements $\xi, \xi^{\prime}$ in $\mathbb{R}^{d}$. We also denote by $\xi \odot \xi^{\prime}$ the Hadamard (or Schur) product of two elements $\xi$ and $\xi^{\prime}$ in $\mathbb{R}^{d}$. For any topological space $\mathbf{T}$, we denote by $\mathcal{B}(\mathbf{T})$ its Borel field.

The paper is concerned with sequences of systems of reaction-diffusion Cauchy problems of the form

$$
(\mathcal{S})\left\{\begin{array}{l}
\frac{d u}{d t}(t)+\partial \Phi_{1}(u(t))=F_{1}(t, u(t), v(t)) \text { for a.e. } t \in(0, T) \\
\frac{d v}{d t}(t)+\partial \Phi_{2}(v(t))=F_{2}(t, u(t), v(t)) \text { for a.e. } t \in(0, T) \\
u(0) \in \overline{\operatorname{dom}\left(\partial \Phi_{1}\right)}, v(0) \in \overline{\operatorname{dom} \partial \Phi_{2}},
\end{array}\right.
$$

where, for $i=1,2, \partial \Phi_{i}$ denote the subdifferential of standard convex functionals $\Phi_{i}$ of the calculus of variations. More precisely $\Phi_{i}: L^{2}(\Omega) \rightarrow \mathbb{R} \cup\{+\infty\}$ is defined by

$$
\Phi_{i}(u)= \begin{cases}\int_{\Omega} W_{i}(x, \nabla u(x)) d x+\frac{1}{2} \int_{\partial \Omega} a_{i} u^{2} d \mathcal{H}_{N-1}-\int_{\partial \Omega} \phi_{i} u d \mathcal{H}_{N-1} & \text { if } u \in H^{1}(\Omega), \\ +\infty & \text { otherwise }\end{cases}
$$

where $a_{i} \in L_{\mathcal{H}_{N-1}^{\infty}}^{\infty}(\partial \Omega)$ with $\left\{\begin{array}{l}a_{i} \geq 0 \quad \mathcal{H}_{N-1} \text {-a.e. in } \partial \Omega \\ \exists \sigma_{i}>0 \quad a_{i} \geq \sigma \quad \mathcal{H}_{N-1} \text {-a.e. in } \Gamma_{i} \subset \partial \Omega \text { with } \mathcal{H}_{N-1}\left(\Gamma_{i}\right)>0,\end{array}\right.$ and $\phi_{i} \in L_{\mathcal{H}_{N-1}}^{2}(\partial \Omega)$.

The density $W_{i}: \mathbb{R}^{N} \times \mathbb{R}^{N} \rightarrow \mathbb{R}$ is a Borel measurable function which satisfies the following conditions:

$\left(D_{1}\right)$ there exist $\alpha>0$ and $\beta>0$ such that for a.e. $x \in \mathbb{R}^{N}$ and every $\xi \in \mathbb{R}^{N}$

$$
\alpha|\xi|^{2} \leq W_{i}(x, \xi) \leq \beta\left(1+|\xi|^{2}\right)
$$

$\left(\mathrm{D}_{2}\right)$ for a.e. $x \in \mathbb{R}^{N}, \xi \mapsto W_{i}(x, \xi)$ is a Gâteaux differentiable 11 convex function (we denote by $D_{\xi} W_{i}(x, \cdot)$ its Gâteaux derivative), with $D_{\xi} W_{i}(x, 0)=0$ for a.e. $x \in \mathbb{R}^{N}$.

By using the subdifferential inequality together with the growth conditions fulfilled by the convex function $\xi \mapsto W_{i}(x, \xi)$, it is easy to show that there exist nonnegative constants $L(\beta)$ and $C(\beta)$ such that, for all $\left(\xi, \xi^{\prime}\right) \in \mathbb{R}^{N} \times \mathbb{R}^{N}$,

$$
\begin{aligned}
& \left|W_{i}(x, \xi)-W_{i}\left(x, \xi^{\prime}\right)\right| \leq L(\beta)\left|\xi-\xi^{\prime}\right|\left(1+|\xi|+\left|\xi^{\prime}\right|\right), \\
& \left|D_{\xi} W(x, \xi)\right| \leq C(\beta)(1+|\xi|) .
\end{aligned}
$$

From the second estimate, we infer that if $u \in H^{1}(\Omega)$, then the function $D_{\xi} W_{i}(\cdot, \nabla u)$ belongs to $L^{2}(\Omega)^{N}$.

We recall (see [1, Lemma 1]) that the subdifferential of the functional $\Phi_{i}$ (actually its Gâteau derivative), whose domain captures the boundary condition, is given by:

$$
\begin{aligned}
& \operatorname{dom}\left(\partial \Phi_{i}\right)=\left\{v \in H^{1}(\Omega): \operatorname{div} D_{\xi} W_{i}(\cdot, \nabla v) \in L^{2}(\Omega), a_{i} v+D_{\xi} W_{i}(\cdot, \nabla v) \cdot \mathbf{n}=\phi_{i} \text { on } \partial \Omega\right\} \\
& \partial \Phi_{i}(v)=-\operatorname{div} D_{\xi} W(\cdot, \nabla v) \text { for } v \in \operatorname{dom}\left(\partial \Phi_{i}\right)
\end{aligned}
$$

where $\mathbf{n}$ denotes the outer unit normal to $\partial \Omega$ and $a_{i} v+D_{\xi} W_{i}(\cdot, \nabla v) \cdot \mathbf{n}$ must be taken in the trace sense. In what follows, since $\partial \Phi_{i}$ are single valued, we denote them by $D \Phi_{i}$.

The pair $\left(F_{1}, F_{2}\right)$ of reaction functionals belongs to a suitable class for which a comparison principle holds with respect to the initial and boundary data for lower and upper solutions. This class is defined in the next section.

\footnotetext{
${ }^{1}$ Under this hypothesis the subdifferential of $\Phi_{i}$ is single valued. We make this hypothesis to simplify the notation.
} 
2.1. The class of TCCP-structured reaction functionals. Reaction-diffusion systems which model a wide class of applications in the domain of ecosystems, and which gives rise to bounded or positive solutions, amenable to analytical calculation in homogenization (periodic or stochastic) involve a special class of pairs of reaction functionals that we define below.

Definition 2.1. A pair $\left(F_{1}, F_{2}\right)$ of functionals $F_{i}:[0,+\infty) \times L^{2}(\Omega) \times L^{2}(\Omega) \rightarrow \mathbb{R}^{\Omega}, i=1,2$, is called a TCCP-structured reaction functional, if there exists a pair of Borel measurable functions $\left(f_{1}, f_{2}\right)$, $f_{i}:[0,+\infty) \times \mathbb{R}^{N} \times \mathbb{R} \times \mathbb{R} \rightarrow \mathbb{R}, i=1,2$, such that for all $t \in[0,+\infty)$ and all $(u, v) \in L^{2}(\Omega) \times L^{2}(\Omega)$,

$$
\left\{\begin{array}{l}
F_{1}(t, u, v)(x)=f_{1}(t, x, u(x), v(x)), \\
F_{2}(t, u, v)(x)=f_{2}(t, x, u(x), v(x)),
\end{array}\right.
$$

which fulfill the following structure conditions:

2 where

$$
\left\{\begin{array}{l}
f_{1}\left(t, x, \zeta, \zeta^{\prime}\right)=r_{1}(t, x) \odot h_{1}\left(\zeta^{\prime}\right) \cdot g_{1}(\zeta)+q_{1}(t, x) \\
f_{2}\left(t, x, \zeta, \zeta^{\prime}\right)=r_{2}(t, x) \odot h_{2}(\zeta) \cdot g_{2}\left(\zeta^{\prime}\right)+q_{2}(t, x)
\end{array}\right.
$$

- $h_{i}, g_{i}: \mathbb{R} \rightarrow \mathbb{R}^{l}, i=1,2$, are locally Lipschitz continuous functions;

- for all $T>0, r_{i} \in L^{\infty}\left([0, T] \times \mathbb{R}^{N}, \mathbb{R}^{l}\right)$;

- for all $T>0, q_{i} \in L^{2}\left(0, T, L_{l o c}^{2}\left(\mathbb{R}^{N}\right)\right)$.

Furthermore $\left(f_{1}, f_{2}\right)$ must satisfy the Two Components Comparison Principle condition $(T C C P)$ : for $i=1,2$, there exists a pair $\left(\underline{f}_{i}, \bar{f}_{i}\right)$ of functions $\underline{f}_{i}, \bar{f}_{i}:[0,+\infty) \times \mathbb{R} \rightarrow \mathbb{R}$ with $\underline{f}_{i} \leq 0 \leq \bar{f}_{i}$, and a pair $\left(\underline{\rho}_{i}, \bar{\rho}_{i}\right)$ in $\mathbb{R}^{2}$ with $\underline{\rho}_{i} \leq \bar{\rho}_{i}$, such that each of the two ordinary differential equations

$$
\underline{O D E_{i}}\left\{\begin{array} { l } 
{ \underline { y } _ { i } ^ { \prime } ( t ) = \underline { f } _ { i } ( t , \underline { y } _ { i } ( t ) ) \text { for a.e.t } \in ( 0 , + \infty ) } \\
{ \underline { y } _ { i } ( o ) = \underline { \rho } _ { i } }
\end{array} \quad \overline { O D E _ { i } } \left\{\begin{array}{l}
\bar{y}_{i}^{\prime}(t)=\bar{f}_{i}\left(t, \bar{y}_{i}(t)\right) \text { for a.e.t } \in(0, \infty) \\
\bar{y}_{i}(o)=\bar{\rho}_{i}
\end{array}\right.\right.
$$

possesses at least one solution, such that for all $T>0$, for a.e. $(t, x) \in(0, T) \times \mathbb{R}^{N}$, we have:

$$
\left\{\begin{array}{l}
f_{1}\left(t, x, \underline{y}_{1}(t), \zeta^{\prime}\right) \geq \underline{f}_{1}\left(t, \underline{y}_{1}(t)\right) \\
f_{1}\left(t, x, \bar{y}_{1}(t), \zeta^{\prime}\right) \leq \bar{f}_{1}\left(t, \bar{y}_{1}(t)\right)
\end{array}\right.
$$

for all $\zeta^{\prime} \in\left[\underline{y}_{2}(T), \bar{y}_{2}(T)\right]$,

and

for all $\zeta \in\left[\underline{y}_{1}(T), \bar{y}_{1}(T)\right]$.

$$
\left\{\begin{array}{l}
f_{2}\left(t, x, \zeta, \underline{y}_{2}(t)\right) \geq \underline{f}_{2}\left(t, \underline{y}_{2}(t)\right) \\
f_{2}\left(t, x, \zeta, \bar{y}_{2}(t)\right) \leq \bar{f}_{2}\left(t, \bar{y}_{2}(t)\right)
\end{array}\right.
$$

The pair $\left(F_{1}, F_{2}\right)$ is called a TCCP-structured reaction functional associated with $\left(r_{i}, g_{i}, h_{i}, q_{i}\right)_{i=1,2}$, and $\left(f_{1}, f_{2}\right)$ a TCCP-structured reaction function associated with $\left(r_{i}, g_{i}, h_{i}, q_{i}\right)_{i=1,2}$.

If furthermore, for all $T>0$, and $i=1,2, r_{i} \in W^{1,1}\left(0, T, L^{2}\left(\mathbb{R}^{N}, \mathbb{R}^{l}\right)\right)$ and $q_{i} \in W^{1,1}\left(0, T, L_{\text {loc }}^{2}\left(\mathbb{R}^{N}\right)\right)$, the pair $\left(F_{1}, F_{2}\right)$ is referred to as a regular TCCP-structured reaction functional and $\left(f_{1}, f_{2}\right)$ as a regular TCCP-structured reaction function.

Remark 2.1. 1) Since $\underline{y}_{i}$ is nonincreasing, and $\bar{y}_{i}$ is nondecreasing, for any $T>0$, and for $i=1,2$ we have

$$
\underline{y}_{i}(T) \leq \underline{y}_{i}(0)=\underline{\rho}_{i}<\bar{\rho}_{i}=\bar{y}_{i}(0) \leq \bar{y}_{i}(T) .
$$

$2)$ It is worth noting that for each fixed $\zeta^{\prime}$ in $\left[\underline{y}_{2}(T) \bar{y}_{2}(T)\right]$, the function $\zeta \mapsto f_{1}\left(t, x, \zeta, \zeta^{\prime}\right)$ is a SVRstructured reaction function associated with $\left(r_{1} \odot h_{1}\left(\zeta^{\prime}\right), g_{1}, q_{1}\right)$ in the sense of [1, Definition 3.1]. Similarly for each fixed $\zeta$ in $\left[\underline{y}_{1}(T) \bar{y}_{1}(T)\right]$, the function $\zeta^{\prime} \mapsto f_{2}\left(t, x, \zeta, \zeta^{\prime}\right)$ is a SVR-structured reaction function associated with $\left(r_{2} \odot h_{2}(\zeta), g_{2}, q_{2}\right)$.

\footnotetext{
${ }^{2}$ Using the coordinates of $r_{i}, g_{i}$ and $h_{i}$ we have $f_{1}\left(t, x, \zeta, \zeta^{\prime}\right)=\sum_{k=1}^{l} r_{1, k}(t, x) h_{1, k}\left(\zeta^{\prime}\right) g_{1, k}(\zeta)+q_{1}(t, x)$ and $f_{2}\left(t, x, \zeta, \zeta^{\prime}\right)=\sum_{k=1}^{l} r_{2, k}(t, x) h_{2, k}(\zeta) g_{2, k}\left(\zeta^{\prime}\right)+q_{2}(t, x)$
} 
2.2. Examples. In examples below, for any $a:[0,+\infty) \times \mathbb{R}^{N} \rightarrow \mathbb{R}$ we use the notation

$$
\bar{a}:=\sup _{(t, x) \in[0,+\infty) \times \mathbb{R}^{N}} a(t, x), \underline{a}:=\inf _{(t, x) \in[0,+\infty) \times \mathbb{R}^{N}} a(t, x) .
$$

The proofs of Propositions below are postponed to Appendix A.

Examples 2.1. Example derived from competition models in ecology.

$$
\begin{aligned}
& f_{1}\left(t, x, \zeta, \zeta^{\prime}\right)=\alpha_{1}(t, x) \zeta\left(1-\frac{\zeta}{K_{1}(t, x)}-a_{1,2} \frac{\zeta^{\prime}}{K_{1}(t, x)}\right) \\
& f_{2}\left(t, x, \zeta, \zeta^{\prime}\right)=\alpha_{2}(t, x) \zeta^{\prime}\left(1-\frac{\zeta^{\prime}}{K_{2}(t, x)}-a_{2,1} \frac{\zeta}{K_{2}(t, x)}\right)
\end{aligned}
$$

where $\alpha_{i}>0$ satisfies $\bar{\alpha}_{i}<+\infty, K_{i}(t, x)>0, a_{1,2}>0$ and $a_{2,1}>0$.

Proposition 2.1. The pair $\left(f_{1}, f_{2}\right)$ is a TCCP-structured reaction function with for $i=1,2$,

$$
\left\{\begin{array}{l}
\underline{\rho}_{i}=0, \underline{y}_{i}=0 \\
\bar{\rho}_{i} \text { is any positif real number, } \bar{y}_{i}(t)=\bar{\rho}_{i} \exp \left(\bar{\alpha}_{i} t\right) .
\end{array}\right.
$$

The pair $\left(f_{1}, f_{2}\right)$ is associated with the diffusive competition model between two species

$$
(\mathcal{S})\left\{\begin{array}{l}
\frac{d u}{d t}(t)+D \Phi_{1}(u(t))=\alpha_{1}(t, \cdot) u(t)\left(1-\frac{u(t)}{K_{1}(t, \cdot)}-a_{1,2} \frac{v(t)}{K_{1}(t, \cdot)}\right) \text { for a.e. } t \in(0, T) \\
\frac{d v}{d t}(t)+D \Phi_{2}(v(t))=\alpha_{2}(t, \cdot) v(t)\left(1-\frac{v(t)}{K_{2}(t, \cdot)}-a_{2,1} \frac{u(t)}{K_{2}(t, \cdot)}\right) \text { for a.e. } t \in(0, T) \\
u(0)=u_{0} \in \overline{\operatorname{dom}\left(D \Phi_{1}\right)}, v(0)=v_{0} \in \overline{\operatorname{dom} D \Phi_{2}},
\end{array}\right.
$$

where $u$ and $v$ denote the densities of two competing species having a logistic growth in the absence of the other. The $\alpha_{i}$ are the birth rates and the $K_{i}$ the carrying capacities. The dimensionless coefficients $a_{1,2}$ and $a_{2,1}$ measure the competing effect of $v$ to $u$ and $u$ to $v$ respectively. In Theorem 2.1 we prove that for all $T>0,(\mathcal{S})$ admits a unique solution $(u, v) \in C\left([0, T], L^{2}(\Omega)\right)^{2}$. Under the initial conditions $0 \leq u_{0} \leq \bar{\rho}_{1}, 0 \leq v_{0} \leq \bar{\rho}_{2}$, this solution fulfills for all $t \in[0, T]$ the bounds $0 \leq u(t) \leq \bar{\rho}_{1} \exp \left(\bar{\alpha}_{1} t\right)$, $0 \leq v(t) \leq \bar{\rho}_{2} \exp \left(\bar{\alpha}_{2} t\right)$. Furthermore, if we assume that the functions $\alpha_{i}, \frac{\alpha_{i}}{K_{i}}$, and $a_{i, \overline{i+1}} \frac{\alpha_{i}(t, x)}{K_{i}(t, x)}$ belong to $W^{1,1}\left(0, T, L_{\text {loc }}^{2}\left(\mathbb{R}^{N}\right)\right) \cap L^{\infty}\left([0, T] \times \mathbb{R}^{N}\right)$, then $u$ and $v$ fulfill the boundary conditions for all $\left.\left.t \in\right] 0, T\right]$ and admit a right derivative at each $t \in] 0, T[$.

Examples 2.2. Example derived from symbiosis models in ecology.

$$
\begin{aligned}
& f_{1}\left(t, x, \zeta, \zeta^{\prime}\right)=\alpha_{1}(t, x) \zeta\left(1-\frac{\zeta}{K_{1}(t, x)}+b_{1,2} \frac{\zeta^{\prime}}{K_{1}(t, x)}\right) \\
& f_{2}\left(t, x, \zeta, \zeta^{\prime}\right)=\alpha_{2}(t, x) \zeta^{\prime}\left(1-\frac{\zeta^{\prime}}{K_{2}(t, x)}+b_{2,1} \frac{\zeta}{K_{2}(t, x)}\right)
\end{aligned}
$$

where $\alpha_{i}>0,+\infty>\bar{K}_{i} \geq K_{i}(t, x) \geq \underline{K}_{i}>0$. We assume that

$$
0 \leq b_{1,2}<\frac{\underline{K}_{1}}{\overline{\bar{K}}_{1}} \text { and } 0 \leq b_{2,1}<\frac{\underline{K}_{2}}{\overline{\bar{K}}_{2}}
$$

Proposition 2.2. The pair $\left(f_{1}, f_{2}\right)$ is a TCCP-structured reaction function with for $i=1,2$,

$$
\left\{\begin{array}{l}
\underline{\rho}_{i}=0, \underline{y}_{i}=0 \\
\bar{\rho}_{1}=\bar{\rho}_{2} \geq \max \left(\frac{\underline{K}_{1} \bar{K}_{1}}{\underline{K}_{1}-b_{1,2} \bar{K}_{1}}, \frac{\underline{K}_{2} \bar{K}_{2}}{\underline{K}_{2}-b_{2,1} \bar{K}_{2}}\right) ; \\
\bar{y}_{i}=\bar{\rho}_{i} .
\end{array}\right.
$$


The pair $\left(f_{1}, f_{2}\right)$ is associated with the diffusive symbiosis model between two species

$$
(\mathcal{S})\left\{\begin{array}{l}
\frac{d u}{d t}(t)+D \Phi_{1}(u(t))=\alpha_{1}(t) u(t)\left(1-\frac{u(t)}{K_{1}(t)}+b_{1,2} \frac{v(t)}{K_{1}(t)}\right) \text { for a.e. } t \in(0, T) \\
\frac{d v}{d t}(t)+D \Phi_{2}(v(t))=\alpha_{2}(t) v(t)\left(1-\frac{v(t)}{K_{2}(t)}+b_{2,1} \frac{u(t)}{K_{2}(t)}\right) \text { for a.e. } t \in(0, T) \\
u(0)=u_{0} \in \overline{\operatorname{dom}\left(D \Phi_{1}\right)}, v_{0}=v_{0} \in \overline{\operatorname{dom} D \Phi_{2}},
\end{array}\right.
$$

where $u$ and $v$ denote the densities of two species having a logistic growth in the absence of the other. Like in Example 2.1, the $\alpha_{i}$ denote the birth rates and the $K_{i}$ the carrying capacities. The dimensionless coefficients $b_{1,2}$ and $b_{2,1}$ measure the symbiosis effect of $v$ to $u$ and $u$ to $v$ respectively. By contrast with the competition model of two species described in Example 2.1. the signs preceding the $b$ 's are positive and reflect the fact that the interaction between the two species is to the advantage of all. Conditions 2.2 reflect the fact that symbiosis between both species must not be too large so that both populations grow while being bounded. Indeed, from Proposition 2.2 , one can choose $0 \leq \bar{\rho}_{1}=\bar{\rho}_{2}<+\infty$. It should be noted that the stability analysis of the system, for the model without diffusion and with constant carrying capacities, provides the less restrictive condition $b_{1,2} b_{2,1}<1$ (see [11, Section 3.6]). In Theorem 2.1 we prove that under the initial conditions $0 \leq u_{0} \leq \bar{\rho}_{1}, 0 \leq v_{0} \leq \bar{\rho}_{2}$, for all $T>0,(\mathcal{S})$ admits a unique solution $(u, v) \in C\left([0, T], L^{2}(\Omega)\right)^{2}$ which fulfills for all $t \in[0, T]$ the bounds $0 \leq u(t) \leq \bar{\rho}_{1}$, and $0 \leq v(t) \leq \bar{\rho}_{2}$. Furthermore, if we assume that the functions $\alpha_{i}, \frac{\alpha_{i}}{K_{i}}$, and $b_{i, \bar{i}+1} \frac{\alpha_{i}(t, x)}{K_{i}(t, x)}$ belong to $W^{1,1}\left(0, T, L_{l o c}^{2}\left(\mathbb{R}^{N}\right)\right) \cap L^{\infty}\left([0, T] \times \mathbb{R}^{N}\right)$, then $u$ and $v$ fulfill the boundary conditions for all $\left.\left.t \in\right] 0, T\right]$ and admit a right derivative at each $t \in] 0, T[$.

Examples 2.3. Example derived from predator-prey models.

$$
\begin{aligned}
& f_{1}\left(t, x, \zeta, \zeta^{\prime}\right)=\alpha_{1}(t, x) \zeta\left(1-\frac{\zeta}{K_{c a r}(t, x)}\right)-a(x, t) \zeta^{\prime}(1-\exp (-b \zeta)) \\
& f_{2}\left(t, x, \zeta, \zeta^{\prime}\right)=\alpha_{2}(t, x) \zeta^{\prime}\left(1-c \frac{\zeta^{\prime}}{\zeta}\right)
\end{aligned}
$$

where $\alpha_{1}(t, x) \geq \underline{\alpha}_{1}>0,+\infty>\bar{\alpha}_{2} \geq \alpha_{2}(t, x) \geq \underline{\alpha}_{2}>0, K_{\text {car }}(t, x) \geq \underline{K}>0,+\infty>\bar{a} \geq a(t, x)>0$, and $b, c$ are positive constants. Furthermore setting $\mu_{\text {ext }}:=c \frac{\underline{\underline{\alpha}}_{1} \underline{\alpha}_{2}}{\overline{a \alpha}_{2}}$, we assume that $\mu_{\text {ext }} \geq 4$.

Proposition 2.3. The pair $\left(f_{1}, f_{2}\right)$ is a TCCP-structured reaction function with

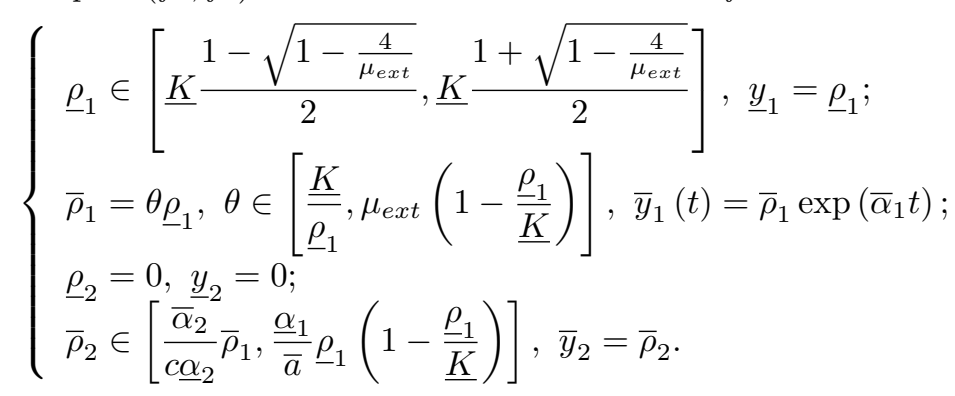

As a consequence of Theorem 2.1, we obtain that under the initial conditions $\underline{\rho}_{1} \leq u_{0} \leq \bar{\rho}_{1}$ and $0 \leq v_{0} \leq \bar{\rho}_{2}$, where $\underline{\rho}_{1}, \bar{\rho}_{1}$ and $\bar{\rho}_{2}$ fulfill condition $(2.3)$, the diffusive predator-prey system

$(\mathcal{S})\left\{\begin{array}{l}\frac{d u}{d t}(t)+D \Phi_{1}(u(t))=\alpha_{1}(t, \cdot) u(t)\left(1-\frac{u(t)}{K_{c a r}(t, \cdot)}\right)-a(t, \cdot) v(t)(1-\exp (-b u(t))) \text { for a.e. } t \in(0, T) \\ \frac{d v}{d t}(t)+D \Phi_{2}(v(t)) \ni \alpha_{2}(t, \cdot) v(t)\left(1-c \frac{v(t)}{u(t)}\right) \text { for a.e. } t \in(0, T) \\ u(0)=u_{0} \in \overline{\operatorname{dom}\left(D \Phi_{1}\right)}, v(0)=v_{0} \in \overline{\operatorname{dom} D \Phi_{2}},\end{array}\right.$

admits for all $T>0$ a unique solution $(u, v) \in C\left([0, T], L^{2}(\Omega)\right)^{2}$ which satisfies for all $t \in[0, T]$, $\underline{\rho}_{1} \leq u(t) \leq \bar{\rho}_{1} \exp \left(\bar{\alpha}_{1}\right)$ and $0 \leq v(t) \leq \bar{\rho}_{2}$. Furthermore, if we assume that the functions $\alpha_{i}, i=$ 
$1,2, \frac{\alpha_{1}}{K_{c a r}}$ and $a$ belong to $W^{1,1}\left(0, T, L_{l o c}^{2}\left(\mathbb{R}^{N}\right)\right) \cap L^{\infty}\left([0, T] \times \mathbb{R}^{N}\right)$, then $u$ and $v$ fulfill the boundary conditions for all $t \in] 0, T]$ and admit a right derivative at each $t \in] 0, T[$. The system models the evolution of two species with density $u$ and $v$ of a prey and a predator, with birth growth rate $\alpha_{1}$ and $\alpha_{2}$ respectively. The prey population satisfies a logistic growth with some time-space depending maximum carrying capacity $K_{c a r}$ (the carrying capacity of the prey when the density of the predator is equal to zero), perturbed by a "predator term" $-a(t, \cdot) v(t)(1-\exp (-b u(t)))$ with a growth coefficient $a$. This term involves a saturation effect, i.e. $-a(t, \cdot) v(t)(1-\exp (-b u(t)))$ saturates to $-a v(t)$ for $u(t)$ large, which reflects the limited capability of the predator when the prey is abundant. There exits many other choice of predator terms with saturation effects, and we refer the reader to [11, Section 3.3] for various examples in the context of o.d.e's. The predator population satisfies a logistic growth with a carrying capacity proportional to the prey density. The condition $\mu_{e x t} \geq 4$ on the dimensionless coefficient $\mu_{\text {ext }}$, prevents the extinction of the prey species since its guarantees existence of $\underline{\rho}_{1}>0$, so that $u(t) \geq \underline{\rho}_{1} \geq \underline{K} \frac{1-\sqrt{1-\frac{4}{\mu_{e x t}}}}{2}$. The coefficient $\mu_{e x t}$ is referred to as the extinction threshold.

Examples 2.4. Example derived from thermo-chimical models.

$$
\begin{aligned}
& f_{1}\left(t, x, \zeta, \zeta^{\prime}\right)=-\alpha_{1}(t, x) \zeta^{p} f_{0}\left(\zeta^{\prime}\right) \\
& f_{2}\left(t, x, \zeta, \zeta^{\prime}\right)=\alpha_{2}(t, x) \zeta^{p} f_{0}\left(\zeta^{\prime}\right)
\end{aligned}
$$

where

$$
f_{0}\left(\zeta^{\prime}\right)=\left\{\begin{array}{l}
\exp \left(\gamma-\frac{\gamma}{\zeta^{\prime}}\right) \text { if } \zeta^{\prime}>0 \\
0 \text { otherwise }
\end{array}\right.
$$

and $\alpha_{i}>0, \bar{\alpha}_{2}<+\infty, p \geq 1$, and $\gamma$ is a positive constant.

Proposition 2.4. The pair $\left(f_{1}, f_{2}\right)$ is a TCCP-structured reaction unction with

$$
\left\{\begin{array}{l}
\rho_{i}=0, \underline{y}_{i}=0 \\
\bar{\rho}_{i} \text { is any positif real number, } \bar{y}_{1}=\bar{\rho}_{1}, \bar{y}_{2}(t)=\bar{\alpha}_{2} \bar{\rho}_{1}^{p} t \exp (\gamma)+\bar{\rho}_{2} .
\end{array}\right.
$$

The pair $\left(f_{1}, f_{2}\right)$ is associated with the diffusive system

$$
(\mathcal{S})\left\{\begin{array}{l}
\frac{d u}{d t}(t)+D \Phi_{1}(u(t))=-\alpha_{1}(t, \cdot) u(t)^{p} f_{0}(v(t)) \text { for a.e. } t \in(0, T) \\
\frac{d v}{d t}(t)+D \Phi_{2}(v(t)) \ni \alpha_{2}(t, \cdot) u(t)^{p} f_{0}(v(t)) \text { for a.e. } t \in(0, T) \\
u(0)=u_{0} \in \overline{\operatorname{dom}\left(D \Phi_{1}\right)}, v(0)=v_{0} \in \overline{\operatorname{dom} D \Phi_{2}},
\end{array}\right.
$$

where $u$ and $v$ denote a chemical concentration and the temperature respectively, in a non isothermal chemical reaction process; $\alpha_{1}$ and $\frac{\alpha_{2}}{\alpha_{1}}$ are called Thiele number and Prater number respectively (see [12] and references therein). In Theorem 2.1 we prove that $(\mathcal{S})$ admits a unique solution $(u, v) \in$ $C\left([0, T], L^{2}(\Omega)\right)^{2}$ under the initial condition $0 \leq u_{0} \leq \bar{\rho}_{1}, 0 \leq v_{0} \leq \bar{\rho}_{2}$, which satisfies the bounds $0 \leq u(t) \leq \bar{\rho}_{1}$, and $0 \leq v(t) \leq \bar{\alpha}_{2} \bar{\rho}_{1}^{p} t \exp (\gamma)+\bar{\rho}_{2}$ for all $t \in[0, T]$. Furthermore, if we assume that the functions $\alpha_{i}$ belong to $W^{1,1}\left(0, T, L_{l o c}^{2}\left(\mathbb{R}^{N}\right)\right) \cap L^{\infty}\left([0, T] \times \mathbb{R}^{N}\right)$, then $u$ and $v$ fulfill the boundary conditions for all $t \in] 0, T]$ and admit a right derivative at each $t \in] 0, T$.

Examples 2.5. Example derived from FitzHugh-Nagumo models.

$$
\begin{aligned}
& f_{1}\left(t, x, \zeta, \zeta^{\prime}\right)=\alpha_{1}(t, x) \zeta(\zeta-a(t, x))(1-\zeta)-b(t, x) \zeta^{\prime} \\
& f_{2}\left(t, x, \zeta, \zeta^{\prime}\right)=\alpha_{2}(t, x) \zeta-c(t, x) \zeta^{\prime}
\end{aligned}
$$

where $\alpha_{1}(t, x) \geq \underline{\alpha}_{1}>0,+\infty>\bar{\alpha}_{2} \geq \alpha_{2}(t, x)>0 ;+\infty>\bar{b} \geq b(t, x) \geq \underline{b}>0 ; c(t, x) \geq \underline{c}>0 ;$ and $0<\underline{a} \leq a(t, x)<1$. 
Proposition 2.5. Set $\gamma=b \frac{\bar{\alpha}_{2}}{\underline{c \alpha}_{1}}$. Then the pair $\left(f_{1}, f_{2}\right)$ is a TCCP-structured reaction function with

$$
\left\{\begin{array}{l}
\bar{\rho}_{1} \geq \max \left(\underline{\gamma}+1, \frac{\sqrt{\frac{\bar{\gamma}}{\underline{\gamma}}}-\underline{a}}{\underline{\gamma}}\right) \\
\underline{\rho}_{1}=-\underline{\gamma} \bar{\rho}_{1} ; \\
\bar{\rho}_{2}=\frac{\bar{\alpha}_{2}}{\underline{c}} \bar{\rho}_{1} ; \\
\underline{\rho}_{2}=\frac{\bar{\alpha}_{2}}{\underline{c}} \underline{\rho}_{1} ; \\
\underline{f}_{i}=\bar{f}_{i}=0, \quad i=1,2 .
\end{array}\right.
$$

The pair $\left(f_{1}, f_{2}\right)$ is associated with the system

$$
(\mathcal{S})\left\{\begin{array}{l}
\frac{d u}{d t}(t)+D \Phi(u(t))=\alpha_{1}(t, \cdot) u(t)(u(t)-a(t, \cdot))(1-u(t))-b(t, \cdot) v(t) \text { for a.e. } t \in(0, T) \\
\frac{d v}{d t}(t)=\alpha_{2}(t, \cdot) u(t)-c(t, \cdot) v(t) \text { for a.e. } t \in(0, T) \\
u(0)=u_{0} \in \overline{\operatorname{dom}(D \Phi)}, v(0)=v_{0},
\end{array}\right.
$$

coupling a reaction diffusion equation with a non diffusive reaction equation. This coupling generalizes the FitzHugh-Nagumo model which describes the evolution of the electrical potential $u$ across the axonal membrane. The variable $v$ is a recovery variable obtained in the simplification of the Hodgkin-Huxley Theory of Nerve Membranes (see [1]). For a complete analysis of boundary value problems relating to FitzHugh-Nagumo equations in one space dimension, we refer the reader to [7, 9, 15. When the initial functions satisfy the bounds $\underline{\rho}_{1} \leq u_{0} \leq \bar{\rho}_{1}$ and $\underline{\rho}_{2} \leq u_{0} \leq \bar{\rho}_{2}$ where $\underline{\rho}_{i}$ and $\bar{\rho}_{i}$ are given by (2.4), existence and uniqueness of solutions fulfilling the same bounds are obtained according to Theorem 2.1 and Remark 2.3 . If we assume that the functions $\alpha_{i}, a, b$, and $c$ belong to $W^{1,1}\left(0, T, L_{\text {loc }}^{2}\left(\mathbb{R}^{N}\right)\right) \cap L^{\infty}\left([0, T] \times \mathbb{R}^{N}\right)$ then $u$ fulfills the boundary condition for all $t \in] 0, T]$ and $u$ and $v$ possess a right derivative at each $t \in] 0, T$. For bounds similar to those given by (2.4), in the case when the coefficients of the reaction functional are constants, we refer the reader to [12, Chapter 12, Section 12.7].

2.3. Existence and uniqueness of a bounded solution. Combining [1, Theorem 3.1] with a suitable fixed point procedure, we establish the existence of a bounded unique solution to the Cauchy problem associated with TCCP-structured reaction functionals.

Theorem 2.1. Let $\Phi_{i}, i=1,2$, be standard functionals of the calculus of variations $(2.1)$ and $\left(F_{1}, F_{2}\right)$ a TCCP-structured reaction functional with $\underline{\rho}_{i}, \bar{\rho}_{i}, \underline{y}_{i}$ and $\bar{y}_{i}$ given by condition $(T C C P)$. Assume that $a_{i} \underline{\rho}_{i} \leq \phi_{i} \leq a_{i} \bar{\rho}_{i}$ for $i=1,2$, then the two component reaction-diffusion system

$$
(\mathcal{S})\left\{\begin{array}{l}
\frac{d u}{d t}(t)+D \Phi_{1}(u(t))=F_{1}(t, u(t), v(t)) \text { for a.e. } t \in(0, T) \\
\frac{d v}{d t}(t)+D \Phi_{2}(v(t))=F_{2}(t, u(t), v(t)) \text { for a.e. } t \in(0, T) \\
\underline{\rho}_{1} \leq u_{0}=u(0) \leq \bar{\rho}_{1}, \quad \underline{\rho}_{2} \leq v_{0}=v(0) \leq \bar{\rho}_{2}, \quad u_{0} \in \overline{\operatorname{dom}\left(D \Phi_{1}\right)}, \quad v_{0} \in \overline{\operatorname{dom} D \Phi_{2}},
\end{array}\right.
$$

admits a unique solution $(u, v) \in C\left([0, T], L^{2}(\Omega)\right) \times C\left([0, T], L^{2}(\Omega)\right)$ satisfying:

$\left(S_{1}\right) u(t) \in \operatorname{dom}\left(D \Phi_{1}\right)$ and $v(t) \in \operatorname{dom}\left(D \Phi_{2}\right)$ for a.e. $t \in(0, T)$,

$\left(S_{2}\right) u$ and $v$ are almost everywhere derivable in $(0, T)$,

$\left(S_{3}\right) u(t) \in\left[\underline{y}_{1}(t), \bar{y}_{1}(t)\right]$ and $v(t) \in\left[\underline{y}_{2}(t), \bar{y}_{2}(t)\right]$ for all $t \in[0, T]$.

If moreover $\left(F_{1}, F_{2}\right)$ is a regular TCCP-structured reaction functional, then $u$ and $v$ satisfy 
$\left(S_{4}\right) u(t) \in \operatorname{dom}\left(D \Phi_{1}\right)$ and $v(t) \in \operatorname{dom}\left(D \Phi_{2}\right)$ for all $\left.\left.t \in\right] 0, T\right]$, $u$ and $v$ possess a right derivative $\frac{d^{+} u}{d t}(t)$ and $\frac{d^{+} v}{d t}(t)$ at every $\left.t \in\right] 0, T[$, and

$$
\left\{\begin{array}{l}
\frac{d^{+} u}{d t}(t)+D \Phi_{1}(u(t))=F_{1}(t, u(t), v(t)), \\
\frac{d^{+} v}{d t}(t)+D \Phi_{1}(v(t))=F_{2}(t, u(t), v(t)) .
\end{array}\right.
$$

Proof. Step 1 (local existence). We prove that there exists a unique solution of $(\mathcal{S})$ for $T$ small enough. For $T>0$ set

$$
X_{T}:=\left\{(u, v) \in C([0, T], X) \times C([0, T], X): u \text { and } v \text { fulfill condition }\left(S_{3}\right)\right\}
$$

which is clearly a closed subset of the space $C([0, T], X) \times C([0, T], X)$ equipped with the norm product defined by $\|(u, v)\|_{C \times C}:=\|u\|_{C([0, T], X)}+\|v\|_{C([0, T], X)}$. Therefore $X_{T}$ is a complete metric space when equipped with the metric associated with the norm $\|\cdot\|_{C \times C}$.

For each $(u, v) \in X_{T}$, we consider the two reaction-diffusion problems with unknown $\Lambda_{1} v$ and $\Lambda_{2} u$ respectively defined by

$$
\begin{aligned}
& \left(\mathcal{P}_{1}\right)\left\{\begin{array}{l}
\frac{d \Lambda_{1} v}{d t}(t)+D \Phi_{1}\left(\Lambda_{1} v(t)\right)=F_{1}\left(t, \Lambda_{1} v(t), v(t)\right) \text { for a.e. } t \in(0, T) \\
\underline{\rho}_{1} \leq \Lambda_{1} v(0)=u_{0} \leq \bar{\rho}_{1},
\end{array}\right. \\
& \left(\mathcal{P}_{2}\right)\left\{\begin{array}{l}
\frac{d \Lambda_{2} u}{d t}(t)+D \Phi_{2}\left(\Lambda_{2} u(t)\right)=F_{2}\left(t, u(t), \Lambda_{2} u(t)\right) \text { for a.e. } t \in(0, T) \\
\underline{\rho}_{2} \leq \Lambda_{2} u(0)=v_{0} \leq \bar{\rho}_{2} .
\end{array}\right.
\end{aligned}
$$

We first claim that $\left(\mathcal{P}_{1}\right)$ and $\left(\mathcal{P}_{2}\right)$ possess a unique solution $\Lambda_{1} v$ and $\Lambda_{2} u$ satisfying $\left(S_{1}\right),\left(S_{2}\right)$ and $\left(S_{3}\right)$ where $\Lambda_{1} v$ and $\Lambda_{2} u$ are substituted for $u$ and $v$ respectively. Indeed, for fixed $(u, v) \in X_{T}$, set

$$
\begin{aligned}
& r_{v}(t, x):=r_{1}(t, x) \odot h_{1}(v(t, x)), f_{v}(t, x, \zeta):=r_{v}(t, x) \cdot g_{1}(\zeta)+q_{1}(t, x), \\
& r_{u}(t, x):=r_{2}(t, x) \odot h_{2}(u(t, x)), f_{u}\left(t, x, \zeta^{\prime}\right):=r_{u}(t, x) \cdot g_{2}\left(\zeta^{\prime}\right)+q_{2}(t, x),
\end{aligned}
$$

and, for $(U, V) \in L^{2}(\Omega) \times L^{2}(\Omega), F_{v}(t, U)(x)=f_{v}(t, x, U(x)), F_{u}(t, V)(x)=f_{u}(t, x, V(x))$. Therefore, $\left(\mathcal{P}_{1}\right)$ and $\left(\mathcal{P}_{2}\right)$ may be written as

$$
\begin{aligned}
& \left(\mathcal{P}_{1}\right)\left\{\begin{array}{l}
\frac{d \Lambda_{1} v}{d t}(t)+D \Phi_{1}\left(\Lambda_{1} v(t)\right)=F_{v}\left(t, \Lambda_{1} v(t)\right) \text { for a.e. } t \in(0, T) \\
\underline{\rho}_{1} \leq \Lambda_{1} v(0)=u_{0} \leq \bar{\rho}_{1},
\end{array}\right. \\
& \left(\mathcal{P}_{2}\right)\left\{\begin{array}{l}
\frac{d \Lambda_{2} u}{d t}(t)+D \Phi_{2}\left(\Lambda_{2} u(t)\right)=F_{u}\left(t, \Lambda_{2} u(t)\right) \text { for a.e. } t \in(0, T) \\
\underline{\rho}_{2} \leq \Lambda_{2} u(0)=v_{0} \leq \bar{\rho}_{2} .
\end{array}\right.
\end{aligned}
$$

The claim follows from [1, Theorem 3.1], provided that we establish that $F_{v}$ and $F_{u}$ are SVR-structured reaction functionals. For this, note that each function $f_{v}$ and $f_{u}$ satisfies the structure condition of SVRstructured reaction functions, and that condition $(C P)$ is fulfilled because $\left(f_{1}, f_{2}\right)$ satisfies $(T C C P)$, and $v$ and $u$ satisfy $\left(S_{3}\right)$.

To show $\left(S_{4}\right)$, it remains to prove that $t \mapsto r_{v}(t, \cdot)$ and $t \mapsto r_{u}(t, \cdot)$ from $[0, T]$ into $L^{2}(\Omega)$ are absolutely continuous. For $t \mapsto r_{v}(t, \cdot)$ the claim follows from the absolute continuity of $r_{1}$ and $v$, and the following estimate

$$
\begin{aligned}
\left\|r_{v}(t, \cdot)-r_{v}(s, \cdot)\right\|_{L^{2}\left(\Omega, \mathbb{R}^{l}\right)} \leq & \left\|r_{1}(t, \cdot) \odot h_{1}(v(t))-r_{1}(s, \cdot) \odot h_{1}(v(t))\right\|_{L^{2}\left(\Omega, \mathbb{R}^{l}\right)} \\
& +\left\|r_{1}(s, \cdot) \odot h_{1}(v(t))-r_{1}(s, \cdot) \odot h_{1}(v(s))\right\|_{L^{2}\left(\Omega, \mathbb{R}^{l}\right)} \\
\leq & \left\|h_{1}\right\|_{L^{\infty}\left(\left[\underline{y}_{2}(T), \bar{y}_{2}(T)\right], \mathbb{R}^{l}\right)}\left\|r_{1}(t, \cdot)-r_{1}(s, \cdot)\right\|_{L^{2}\left(\Omega, \mathbb{R}^{l}\right)} \\
& +\left\|r_{1}\right\|_{L^{\infty}\left([0, T] \times \mathbb{R}^{N}, \mathbb{R}^{l}\right)} L_{h_{1}}\|v(t)-v(s)\|_{L^{2}(\Omega)}
\end{aligned}
$$

where $L_{h_{1}}$ denotes the Lipschitz constant of $h_{1}$ in $\left[\underline{y}_{2}(T), \bar{y}_{2}(T)\right]$. For $t \mapsto r_{u}(t, \cdot)$ the proof is similar. 
Let us consider the operator $\Lambda: X_{T} \rightarrow C([0, T], X) \times C([0, T], X)$ defined by $\Lambda(u, v)=\left(\Lambda_{1} v, \Lambda_{2} u\right)$. We are going to establish existence of a fixed point of $\Lambda$ for $T>0$ small enough. Such a fixed point clearly furnishes a solution of $(\mathcal{S})$ fulfilling $\left(S_{1}\right)-\left(S_{4}\right)$.

We claim that $\Lambda\left(X_{T}\right) \subset X_{T}$. Let $(u, v) \in X_{T}$, then $\Lambda(u, v)=\left(\Lambda_{1} v, \Lambda_{2} u\right)$. According to the considerations above, as $\Lambda_{1} v$ and $\Lambda_{2} u$ solve $\left(\mathcal{P}_{1}\right)$ and $\left(\mathcal{P}_{2}\right)$ respectively, we have $\left(\Lambda_{1} v, \Lambda_{2} u\right) \in C([0, T], X) \times$ $C([0, T], X)$, and $\underline{y}_{1}(t) \leq \Lambda_{1} v(t) \leq \bar{y}_{1}(t), \underline{y}_{2}(t) \leq \Lambda_{2} u(t) \leq \bar{y}_{2}(t)$. Therefore $\left(\Lambda v, \Lambda_{2} u\right)$ belongs to $X_{T}$. We claim that $\Lambda$ is a contraction for $T>0$ small enough. Let $\left(u_{1}, v_{1}\right)$ and $\left(u_{2}, v_{2}\right)$ in $X_{T}$. We first estimate

$$
\left\|\Lambda\left(u_{1}, v_{1}\right)-\Lambda\left(u_{2}, v_{2}\right)\right\|_{C \times C}=\left\|\left(\Lambda_{1} v_{1}-\Lambda_{1} v_{2}\right)\right\|_{X}+\left\|\left(\Lambda_{2} u_{1}-\Lambda_{2} u_{2}\right)\right\|_{X}
$$

From $\left(\mathcal{P}_{1}\right)$, subtract the equation related to $\Lambda_{1} v_{1}$ from the equation related to $\Lambda_{1} v_{2}$ and take the scalar product in $\mathrm{X}$ with $\Lambda_{1} v_{1}-\Lambda_{1} v_{2}$. Using the fact that $D \Phi_{1}$ is a monotone operator, we obtain that for a.e. $t \in(0, T)$

$$
\frac{1}{2} \frac{d}{d t}\left\|\left(\Lambda_{1} v_{1}-\Lambda_{1} v_{2}\right)(t)\right\|_{X}^{2} \leq\left\langle F_{1}\left(t, \Lambda_{1} v_{1}(t), v_{1}(t)\right)-F_{1}\left(t, \Lambda_{1} v_{2}(t), v_{2}(t)\right), \Lambda_{1} v_{1}(t)-\Lambda_{1} v_{2}(t)\right\rangle .
$$

Thus, for a.e. $t \in(0, T)$,

$$
\begin{aligned}
\frac{d}{d t}\left\|\left(\Lambda_{1} v_{1}-\Lambda_{1} v_{2}\right)(t)\right\|_{X}^{2} & \leq 2\left\|F_{1}\left(t, \Lambda_{1} v_{1}(t), v_{1}(t)\right)-F_{1}\left(t, \Lambda_{1} v_{2}(t), v_{2}(t)\right)\right\|_{X}\left\|\Lambda_{1} v_{1}(t)-\Lambda_{1} v_{2}(t)\right\|_{X} \\
& \leq\left\|F_{1}\left(t, \Lambda_{1} v_{1}(t), v_{1}(t)\right)-F_{1}\left(t, \Lambda_{1} v_{2}(t), v_{2}(t)\right)\right\|_{X}^{2}+\left\|\Lambda_{1} v_{1}(t)-\Lambda_{1} v_{2}(t)\right\|_{X}^{2}
\end{aligned}
$$

According to the structure of the functional $F_{1}$, we have

$$
\begin{aligned}
& \left\|F_{1}\left(t, \Lambda_{1} v_{1}(t), v_{1}(t)\right)-F_{1}\left(t, \Lambda_{1} v_{2}(t), v_{2}(t)\right)\right\|_{X}^{2} \\
\leq \quad & C\left(T, g_{1}, h_{1}\right)\left\|v_{1}(t)-v_{2}(t)\right\|_{X}^{2}+C^{\prime}\left(T, g_{1}, h_{1}\right)\left\|\Lambda_{1} v_{1}(t)-\Lambda_{1} v_{2}(t)\right\|_{X}^{2}
\end{aligned}
$$

with

$$
\begin{aligned}
& C\left(T, g_{1}, h_{1}\right)=2 \sup _{\zeta \in\left[\underline{y}_{1}(T), \bar{y}_{1}(T)\right]}\left|g_{1}(\zeta)\right|^{2}\left\|r_{1}\right\|_{L^{\infty}\left(\mathbb{R}^{N}, \mathbb{R}^{l}\right)}^{2} L_{h_{1}, T}, \\
& C^{\prime}\left(T, g_{1}, h_{1}\right)=2 \sup _{\zeta^{\prime} \in\left[\underline{y}_{2}(T), \bar{y}_{2}(T)\right]}\left|h_{1}\left(\zeta^{\prime}\right)\right|^{2}\left\|r_{1}\right\|_{L^{\infty}\left(\mathbb{R}^{N}, \mathbb{R}^{l}\right)}^{2} L_{g_{1}, T},
\end{aligned}
$$

where $L_{g_{1}, T}, L_{h_{1}, T}$ denote the Lipschitz constants of the restrictions of $g_{1}$ and $h_{1}$ on $\left[\underline{y}_{1}(T), \bar{y}_{1}(T)\right]$ and $\left[\underline{y}_{2}(T), \bar{y}_{2}(T)\right]$ respectively. Combining 2.5 and 2.6 we infer that for a.e. $t \in(0, T)$

$$
\begin{aligned}
\frac{d}{d t}\left\|\left(\Lambda_{1} v_{1}(t)-\Lambda_{1} v_{2}(t)\right)\right\|_{X}^{2} & \leq C\left(T, g_{1}, h_{1}\right)\left\|v_{1}(t)-v_{2}(t)\right\|_{X}^{2} \\
& +\left(1+C^{\prime}\left(T, g_{1}, h_{1}\right)\right)\left\|\Lambda_{1} v_{1}(t)-\Lambda_{1} v_{2}(t)\right\|_{X}^{2} .
\end{aligned}
$$

By integrating this inequality over $(0, s)$ for $s \in[0, T]$ and noticing that $\Lambda_{1} v_{1}(0)=\Lambda_{1} v_{2}(0)=u_{0}$, we obtain

$$
\begin{aligned}
\left\|\Lambda_{1} v_{1}(s)-\Lambda_{1} v_{2}(s)\right\|_{X}^{2} & \leq C\left(T, g_{1}, h_{1}\right) \int_{0}^{s}\left\|v_{1}(t)-v_{2}(t)\right\|_{X}^{2} d t \\
& +\left(1+C^{\prime}\left(T, g_{1}, h_{1}\right)\right) \int_{0}^{s}\left\|\Lambda_{1} v_{1}(t)-\Lambda_{1} v_{2}(t)\right\|_{X}^{2} d t
\end{aligned}
$$

from which, according to Grönwall's lemma, we deduce that for all $s \in[0, T]$,

$$
\left\|\left(\Lambda_{1} v_{1}(s)-\Lambda_{1} v_{2}(s)\right)\right\|_{X}^{2} \leq T C\left(T, g_{1}, h_{1}\right)\left\|v_{1}-v_{2}\right\|_{C([0, T], X)}^{2} \exp \left(\left(1+C^{\prime}\left(T, g_{1}, h_{1}\right)\right) T\right) .
$$

Proceeding similarly, we obtain, with suitable adapted notation,

$$
\left\|\left(\Lambda_{2} u_{1}(s)-\Lambda_{2} u_{2}(s)\right)\right\|_{X}^{2} \leq T C\left(T, g_{2}, h_{2}\right)\left\|u_{1}-u_{2}\right\|_{C([0, T], X)}^{2} \exp \left(\left(1+C^{\prime}\left(T, g_{2}, h_{2}\right)\right) T\right) .
$$

Consequently

$$
\left\|\Lambda\left(u_{1}, v_{1}\right)-\Lambda\left(u_{2}, v_{2}\right)\right\|_{C \times C} \leq C(T)\left\|\left(u_{1}, v_{1}\right)-\left(u_{2}, v_{2}\right)\right\|_{C \times C}
$$

where

$C(T)=T \max \left(C\left(T, g_{1}, h_{1}\right)^{\frac{1}{2}} \exp \left(\left(1+C^{\prime}\left(T, g_{1}, h_{1}\right)\right) \frac{T}{2}\right), C\left(T, g_{2}, h_{2}\right)^{\frac{1}{2}} \exp \left(\left(1+C^{\prime}\left(T, g_{2}, h_{2}\right)\right) \frac{T}{2}\right)\right)$. 
For $i=1,2$, the nonnegative constants $C\left(T, g_{i}, h_{i}\right)$ and $C^{\prime}\left(T, g_{i}, h_{i}\right)$ are clearly nondecreasing so that $\lim _{T \rightarrow 0} C(T)=0$. Consequently $\Lambda$ is a contraction for $T$ small enough and admits a fixed point $(u, v)$, i.e. $\left(\Lambda_{1} v, \Lambda_{2} u\right)=(u, v)$ so that $\Lambda_{1} v=u$ and $\Lambda_{2} u=v$. This proves that $(u, v)$ solves $(\mathcal{S})$.

Step 2 (uniqueness). Let $\left(u_{1}, v_{1}\right)$ and $\left(u_{2}, v_{2}\right)$ be two solutions of $(\mathcal{S})$, then taking $\Lambda_{1} v_{1}=u_{1}$ and $\Lambda_{1} v_{2}=u_{2}$ in 2.8, we infer that for all $s \in[0, T]$

$$
\left\|u_{1}(s)-u_{2}(s)\right\|_{X}^{2} \leq C\left(T, g_{1}, h_{1}\right) \int_{0}^{s}\left\|v_{1}(t)-v_{2}(t)\right\|_{X}^{2} d t+\left(1+C^{\prime}\left(T, g_{1}, h_{1}\right)\right) \int_{0}^{s}\left\|u_{1}(t)-u_{2}(t)\right\|_{X}^{2} d t
$$

similarly

$$
\left\|v_{1}(s)-v_{2}(s)\right\|_{X}^{2} \leq C\left(T, g_{2}, h_{2}\right) \int_{0}^{s}\left\|u_{1}(t)-u_{2}(t)\right\|_{X}^{2} d t+\left(1+C^{\prime}\left(T, g_{2}, h_{2}\right)\right) \int_{0}^{s}\left\|v_{1}(t)-v_{2}(t)\right\|_{X}^{2} d t
$$

By summing these two inequalities, we obtain for a.e. $s \in[0, T]$,

$$
\left\|u_{1}(s)-u_{2}(s)\right\|_{X}^{2}+\left\|v_{1}(s)-v_{2}(s)\right\|_{X}^{2} \leq C \int_{0}^{s}\left(\left\|u_{1}(t)-u_{2}(t)\right\|_{X}^{2}+\left\|v_{1}(t)-v_{2}(t)\right\|_{X}^{2}\right) d t
$$

for some nonnegative constant $C$. Hence, according to Grönwall's Lemma, for all $s \in[0, T]$,

$$
\left\|u_{1}(s)-u_{2}(s)\right\|_{X}^{2}+\left\|v_{1}(s)-v_{2}(s)\right\|_{X}^{2}=0
$$

which proves uniqueness.

Step 3 (existence of a global solution). Denote by $T^{*}>0$ a small enough number obtained in Step 1 so that $(\mathcal{S})$ admits a unique solution in $\left.C\left(\left[0, T^{*}\right], X\right) \times C\left(\left[0, T^{*}\right], X\right)\right]^{3}$, By 4 , Theorem 17.2.5] or [5. Theorem 3.6]), we have $\sqrt{t} \frac{d u}{d t} \in L^{2}\left(0, T^{*}, X\right)$. Hence, for $0<\delta<T^{*}$, $\frac{d u}{d t}$ belongs to $L^{2}\left(\delta, T^{*}, X\right)$. Set

$$
E:=\{T>\delta: \exists(u, v) \in C([0, T], X) \times C([0, T], X) \text { solution of }(\mathcal{S})\} .
$$

Since $T^{*} \in E$, we have $E \neq \emptyset$. Set $T_{M a x}:=\sup E$ in $\overline{\mathbb{R}}_{+}$and denote by $(u, v)$ the maximal solution of $(\mathcal{S})$ in $C\left(\left[0, T_{\operatorname{Max}}\right), X\right) \times C\left(\left[0, T_{\operatorname{Max}}\right), X\right)$. We argue by contradiction assuming that $T_{\text {Max }}<+\infty$.

a) We first prove the existence of the two limits $\lim _{t \rightarrow T_{\text {Max }}} u(t)$ and $\lim _{t \rightarrow T_{M a x}} v(t)$ in $X$.

Let $T \in E$, then for a.e. $t \in(0, T)$ we have

$$
\begin{aligned}
& \left\langle\frac{d u}{d t}(t), \frac{d u}{d t}(t)\right\rangle+\left\langle D \Phi_{1} u(t), \frac{d u}{d t}(t)\right\rangle=\left\langle F_{1}(t, u(t), v(t)), \frac{d u}{d t}(t)\right\rangle, \\
& \left\langle\frac{d v}{d t}(t), \frac{d v}{d t}(t)\right\rangle+\left\langle D \Phi_{2} v(t), \frac{d v}{d t}(t)\right\rangle=\left\langle F_{2}(t, u(t), v(t)), \frac{d v}{d t}(t)\right\rangle .
\end{aligned}
$$

From 2.9$]$, we infer that

$$
\begin{aligned}
& \int_{\delta}^{T}\left\|\frac{d u}{d t}(t)\right\|_{X}^{2} d t+\Phi_{1}(u(t))-\Phi_{1}(u(\delta)) \\
& \leq\left(\int_{0}^{T}\left\|F_{1}(t, u(t), v(t))\right\|_{X}^{2} d t\right)^{\frac{1}{2}}\left(\int_{\delta}^{T}\left\|\frac{d u}{d t}(t)\right\|_{X}^{2} d t\right)^{\frac{1}{2}} .
\end{aligned}
$$

For all $T \in E$, we have $\left[\underline{y}_{1}(T), \bar{y}_{1}(T)\right] \subset\left[\underline{y}_{1}\left(T_{\max }\right), \bar{y}_{1}\left(T_{\max }\right)\right]$, and $\left[\underline{y}_{2}(T), \bar{y}_{2}(T)\right] \subset\left[\underline{y}_{2}\left(T_{\max }\right), \bar{y}_{2}\left(T_{\max }\right)\right]$. Thus, according to the structure of $F_{1}$, there exists a constant

$$
C=C\left(\left\|r_{1}\right\|_{L^{\infty}\left(\mathbb{R}^{N}, \mathbb{R}^{l}\right)},\left\|g_{1}\right\|_{L^{\infty}\left(\left[\underline{y}_{1}\left(T_{\max }\right), \bar{y}_{1}\left(T_{\max }\right)\right], \mathbb{R}^{l}\right)},\left\|h_{1}\right\|_{L^{\infty}\left(\left[\underline{y}_{2}\left(T_{\max }\right), \bar{y}_{2}\left(T_{\max }\right)\right], \mathbb{R}^{l}\right)}\right)
$$

such that

Therefore, since

$$
\left\|F_{1}(t, u(t), v(t))\right\|_{X}^{2} \leq 2 C^{2} \mathcal{L}_{N}(\Omega)+2\left\|q_{1}(t, \cdot)\right\|_{X}^{2}
$$

$$
\inf _{v \in L^{2}(\Omega)} \Phi_{1}(w) \geq-\frac{C_{\text {trace }}}{2 \nu}\left\|\phi_{1}\right\|_{L_{\mathcal{H}_{N-1}}^{2}}^{2}(\partial \Omega)
$$

\footnotetext{
${ }^{3}$ Recall that under the initial condition $u_{0} \in \overline{\operatorname{dom}\left(D \Phi_{1}\right)}$ we are not assured that the derivative $\frac{d u}{d t}$ of the solution belongs to $L^{2}\left(0, T^{*}, X\right)$.
} 
(for a proof refer to [2]), and $q_{1} \in L^{2}\left(0, T_{\max }, L^{2}(\Omega)\right)$, inequality 2.11 yields

$$
\int_{\delta}^{T}\left\|\frac{d u}{d t}(t)\right\|_{X}^{2} d t \leq C\left(1+\left(\int_{\delta}^{T}\left\|\frac{d u}{d t}(t)\right\|_{X}^{2} d t\right)^{\frac{1}{2}}\right)
$$

where the new constant $C$ does not depend on $T$. We infer that

$$
\int_{\delta}^{T_{\text {Max }}}\left\|\frac{d u}{d t}(t)\right\|_{X}^{2} d t=\sup _{T \in E} \int_{\delta}^{T}\left\|\frac{d u}{d t}(t)\right\|_{X}^{2} d t<+\infty,
$$

from which we deduce that $u:\left[\delta, T_{\operatorname{Max}}\right) \rightarrow X$ is uniformly continuous. Indeed, for $s<t$ in $\left[\delta, T_{\operatorname{Max}}\right)$ we have

$$
\|u(t)-u(s)\|_{X} \leq \int_{s}^{t}\left\|\frac{d u}{d \tau}(\tau)\right\|_{X}^{2} d \tau \leq(t-s)^{\frac{1}{2}}\left(\int_{\delta}^{T_{M a x}}\left\|\frac{d u}{d \tau}(t)\right\|_{X}^{2} d t\right)^{\frac{1}{2}}
$$

and $u$ is more precisely $\frac{1}{2}$-Holder continuous. Since $X$ is a complete normed space, according to the continuous extension principle, $u$ possesses a unique continuous extension $\bar{u}$ in $\left[\delta, T_{\text {Max }}\right]$ i.e. $\lim _{t \rightarrow T_{\text {Max }}} u(t)=$ $\bar{u}\left(T_{\text {Max }}\right)$. Similarly, from 2.10, we deduce that $v$ possesses a unique continuous extension $\bar{v}$ in $\left[\delta, T_{\text {Max }}\right]$ i.e. $\lim _{t \rightarrow T_{M a x}} v(t)=\bar{v}\left(T_{\text {Max }}\right)$, which proves the claim.

b) Contradiction: For $T>0$, consider the two component reaction-diffusion system

$$
\left(\mathcal{S}^{\prime}\right)\left\{\begin{array}{l}
\frac{d U}{d t}(t)+D \Phi_{1}(U(t))=F_{1}(t, U(t), V(t)) \text { for a.e. } t \in(0, T) \\
\frac{d V}{d t}(t)+D \Phi_{2}(V(t))=F_{2}(t, U(t), V(t)) \text { for a.e. } t \in(0, T) \\
U(0)=\bar{u}\left(T_{\max }\right), V(0)=\bar{v}\left(T_{\max }\right) \\
\underline{\rho}_{1}^{\prime} \leq U(0) \leq \bar{\rho}_{1}^{\prime}, \quad \underline{\rho}_{2}^{\prime} \leq V(0) \leq \bar{\rho}_{2}^{\prime}
\end{array}\right.
$$

where $\underline{\rho}_{1}^{\prime}=\underline{y}_{1}\left(T_{\max }\right), \bar{\rho}_{1}^{\prime}=\bar{y}_{1}\left(T_{\max }\right)$, and $\underline{\rho}_{2}^{\prime}=\underline{y}_{2}\left(T_{\max }\right), \bar{\rho}_{2}^{\prime}=\bar{y}_{2}\left(T_{\max }\right)$. Note that $U(0) \in$ $\overline{\operatorname{dom}\left(D \Phi_{1}\right)}$ and $V(0) \in \overline{\operatorname{dom}\left(D \Phi_{2}\right)}$. Then according to step 1, there exists $T^{* *}>0$ small enough such that $\left(\mathcal{S}^{\prime}\right)$ possesses a solution $(U, V) \in C\left(\left[0, T^{* *}\right], X\right) \times C\left(\left[0, T^{* *}\right], X\right)$. Set

$$
\tilde{u}(t)=\left\{\begin{array}{l}
u(t) \text { if } t \in\left[0, T_{\text {Max }}\right] \\
U\left(t-T_{\text {Max }}\right) \text { if } t \in\left[T_{\text {Max }}, T_{\text {Max }}+T^{* *}\right],
\end{array}\right.
$$

and

$$
\widetilde{v}(t)=\left\{\begin{array}{l}
v(t) \text { if } t \in\left[0, T_{\text {Max }}\right] \\
V\left(t-T_{\text {Max }}\right) \text { if } t \in\left[T_{\text {Max }}, T_{\text {Max }}+T^{* *}\right] .
\end{array}\right.
$$

Then $(\widetilde{u}, \widetilde{v}) \in C\left(\left[0, T_{\text {Max }}+T^{* *}\right], X\right) \times C\left(\left[0, T_{\text {Max }}+T^{* *}\right], X\right)$ is a solution of $(\mathcal{S})$, which leads to a contradiction with the maximality of $T_{\text {Max }}$.

Remark 2.2. By using [1, Corollary 3.2], and arguing as in the proof above, the conclusion of Theorem 2.1 still holds if for $i=1$ or $i=2$, the functional $\Phi_{i}$ is of the form

$$
\Phi_{i}(u)= \begin{cases}\int_{\Omega} W_{i}(x, \nabla u(x)) d x & \text { if } u \in H_{\Gamma_{i}}^{1}(\Omega) \\ +\infty & \text { otherwise }\end{cases}
$$

and $\underline{\rho}_{i} \leq 0 \leq \bar{\rho}_{i}$ (recall that $H_{\Gamma_{i}}^{1}(\Omega)=\left\{v \in H_{1}(\Omega): u=0\right.$ on $\left.\Gamma_{i}\right\}$. The domain of $\partial \Phi_{i}$ contains the Dirichlet-Neumann boundary conditions as stated in [1, Lemma 3.2]:

$\operatorname{dom}\left(\partial \Phi_{i}\right)=\left\{w \in H(\Omega): \operatorname{div} D_{\xi} W_{i}(\cdot, \nabla w) \in L^{2}(\Omega), w=0\right.$ on $\Gamma_{i}, D_{\xi} W_{i}(\cdot, \nabla w) \cdot \eta=0$ on $\left.\partial \Omega \backslash \Gamma_{i}\right\}$.

Remark 2.3. A careful analysis of the proof of [1, Corollary 3.1] shows that its conclusion still holds when $\Phi_{i}=0$ for $i=1$ or $i=2$ (in the sense $W_{i}=a_{i}=\phi_{i}=0$ ). Indeed the lower condition $\alpha|\xi|^{2} \leq W_{i}(x, \xi)$ with $\alpha>0$, only serves to ensure that $\inf _{v \in L^{2}(\Omega)} \Phi_{i}(v)>-\infty$. Therefore Theorem 2.1 remains valid for systems $(\mathcal{S})$ coupling a reaction-diffusion equation (r.d.e.) with a non diffusive reaction equation (n.d.r..e.) (see Example 2.5), or two non diffusive reaction equations (n.d.r.e..). 


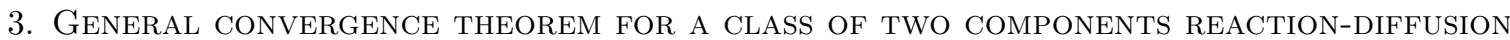 SYSTEMS}

For each $i=1,2$, let $\left(\Phi_{i, n}\right)_{n \in \mathbb{N}}$ be a sequence of functional of the calculus of variations where $\Phi_{i, n}$ : $L^{2}(\Omega) \rightarrow \mathbb{R} \cup\{+\infty\}$ is defined by

$$
\Phi_{i, n}(u)= \begin{cases}\int_{\Omega} W_{n}(x, \nabla u(x)) d x+\frac{1}{2} \int_{\partial \Omega} a_{i, n} u^{2} d \mathcal{H}_{N-1}-\int_{\partial \Omega} \phi_{i, n} u d \mathcal{H}_{N-1} & \text { if } u \in H^{1}(\Omega), \\ +\infty & \text { otherwise }\end{cases}
$$

We assume that $\phi_{i, n} \in L_{\mathcal{H}_{N-1}}^{2}(\partial \Omega), a_{i, n} \in L_{\mathcal{H}_{N-1}}^{\infty}(\partial \Omega)$ with $a_{i, n} \geq 0 \mathcal{H}_{N-1}$ a.e. in $\partial \Omega$, and $a_{i, n} \geq \sigma_{i, n}$ on $\Gamma_{i} \subset \partial \Omega$ with $\mathcal{H}_{N-1}\left(\Gamma_{i}\right)>0$ for some $\sigma_{i, n}>0$ and that $W_{i, n}: \mathbb{R}^{N} \times \mathbb{R}^{N} \rightarrow \mathbb{R}$ is a Borel measurable function which fulfills the following conditions:

$\left(D_{1, n}\right)$ there exist $\left\{\alpha_{i, n}\right\} \subset \mathbb{R}_{+}^{*}$ and $\left\{\beta_{i, n}\right\} \subset \mathbb{R}_{+}^{*}$, such that for a.e. $x \in \mathbb{R}^{N}$ and all $\xi \in \mathbb{R}^{N}$ and all $n \in \mathbb{N}$,

$$
\alpha_{i, n}|\xi|^{2} \leq W_{i, n}(x, \xi) \leq \beta_{i, n}\left(1+|\xi|^{2}\right),
$$

$\left(\mathrm{D}_{2, n}\right)$ for a.e. $x \in \mathbb{R}^{N}, \xi \mapsto W_{i, n}(x, \xi)$ is a differentiable and convex function, and $D_{\xi} W_{i, n}(x, 0)=0$,

$\left(\mathrm{D}_{3, n}\right) \exists \gamma_{i}>0$, s. t. for all $\xi \in \mathbb{R}^{N}, \inf _{n \in \mathbb{N}} \inf _{x \in \mathbb{R}^{N}} D_{\zeta} W_{i, n}(x, \xi) . \xi \geq \gamma_{i}|\xi|^{2}$.

In the following we fix $T>0$ and consider a sequence $\left(\left(F_{1, n}, F_{2, n}\right)\right)_{n \in \mathbb{N}}$ of TCSVR-functionals, each of them being associated with $\left(r_{i, n}, g_{i, n}, h_{i, n}, q_{i, n}\right)$, i.e. $F_{i, n}(t, u, v)(x)=f_{i, n}(t, x, u(x) v(x))$ for all $t \in[0, T]$, a.e. $x \in \Omega$, and all $(u, v) \in L^{2}(\Omega)^{2}$, where

$$
\begin{aligned}
& f_{1, n}\left(t, x, \zeta, \zeta^{\prime}\right)=r_{1, n}(t, x) \odot h_{i, n}\left(\zeta^{\prime}\right) \cdot g_{1, n}(\zeta)+q_{1, n}(t, x) \text { for all }(t, x, \zeta) \in[0,+\infty) \times \mathbb{R}^{N} \times \mathbb{R} \\
& f_{2, n}\left(t, x, \zeta, \zeta^{\prime}\right)=r_{2, n}(t, x) \odot h_{2, n}(\zeta) \cdot g_{2, n}\left(\zeta^{\prime}\right)+q_{2, n}(t, x) \text { for all }(t, x, \zeta) \in[0,+\infty) \times \mathbb{R}^{N} \times \mathbb{R} .
\end{aligned}
$$

We assume that for all $n \in \mathbb{N}, h_{i, n}$ and $g_{i, n}$ are locally Lipschitz functions, uniformly with respect to $n$, i.e. for all interval $I \subset \mathbb{R}$, there exists $L_{I} \geq 0$ and $L_{I}^{\prime} \geq 0$ such that

$$
\begin{aligned}
& \sup _{n \in \mathbb{N}}\left|g_{i, n}(\zeta)-g_{i, n}\left(\zeta^{\prime}\right)\right| \leq L_{I}\left|\zeta-\zeta^{\prime}\right|, \forall\left(\zeta, \zeta^{\prime}\right) \in \mathbb{R}^{2}, \\
& \sup _{n \in \mathbb{N}}\left|h_{i, n}(\zeta)-h_{i, n}\left(\zeta^{\prime}\right)\right| \leq L_{I}^{\prime}\left|\zeta-\zeta^{\prime}\right|, \forall\left(\zeta, \zeta^{\prime}\right) \in \mathbb{R}^{2} .
\end{aligned}
$$

The functions $r_{i, n}$ and $q_{i, n}$ are uniformly absolutely continuous, i.e.

$$
\begin{aligned}
& \sup _{n} \int_{0}^{T}\left\|\frac{d r_{i, n}}{d t}(t, \cdot)\right\|_{L^{2}\left(\Omega, \mathbb{R}^{l}\right)} d t<+\infty, \\
& \sup _{n} \int_{0}^{T}\left\|\frac{d q_{i, n}}{d t}(t, \cdot)\right\|_{L^{2}(\Omega)} d t<+\infty .
\end{aligned}
$$

We finally assume that

$$
\underline{\rho}_{i}:=\inf _{n} \underline{y}_{i, n}(T)>-\infty \text { and } \bar{\rho}_{i}:=\sup _{n} \bar{y}_{i, n}(T)<+\infty,
$$

and, for all $n \in \mathbb{N}$,

$$
a_{i, n} \underline{\rho}_{i, n} \leq \phi_{i, n} \leq a_{i, n} \bar{\rho}_{i, n} \text { on } \partial \Omega
$$

where for $i=1,2, \underline{y}_{i, n}, \bar{y}_{i, n}, \underline{\rho}_{i, n}$ and $\bar{\rho}_{i, n}$ are given by condition $(T C C P)$ fulfilled by $\left(F_{1, n}, F_{2, n}\right)$. Recall that $\underline{y}_{i, n}$ and $\bar{y}_{i, n}$ are solution of $\underline{O D E_{i}}$ and $\overline{O D E_{i}}$ with $\underline{f}_{i, n}, \bar{f}_{i, n}$ and initial condition $\underline{\rho}_{i, n}$ and $\bar{\rho}_{i, n}$ respectively.

Recall that a sequence $\left(\Phi_{n}\right)_{n \in \mathbb{N}}$ of lower semicontinuous convex proper functionals $\Phi_{n}: L^{2}(\Omega) \rightarrow$ $\mathbb{R} \cup\{+\infty\}$ Mosco-converges to a functional $\Phi$ if $\left(\Phi_{n}\right)_{n \in \mathbb{N}} \Gamma$-converges to $\Phi$ when the $\Gamma$-convergence is associated both with the strong and the weak topology of $L^{2}(\Omega)$. We write $\Phi_{i, n} \stackrel{M}{\rightarrow} \Phi_{i}$. For details consult Appendix $\mathrm{C}$ and references therein.

Theorem 3.1 (General convergence theorem). Assume that for $i=1,2$, the sequence $\left(W_{i, n}\right)_{n \in \mathbb{N}}$ satisfies conditions $\left(\mathrm{D}_{1, n}\right),\left(\mathrm{D}_{2, n}\right),\left(\mathrm{D}_{3, n}\right)$, and that the sequence of TCCP-structured reaction functionals 
$\left(F_{1, n}, F_{2, n}\right)_{n \in \mathbb{N}}$ satisfies conditions (3.2) (3.3), 3.4), 3.5). Let $\left(u_{n}, v_{n}\right)$ be the unique solution of the system

$$
\left(\mathcal{S}_{n}\right)\left\{\begin{array}{l}
\frac{d u_{n}}{d t}(t)+D \Phi_{1, n}\left(u_{n}(t)\right)=F_{1, n}\left(t, u_{n}(t), v_{n}(t)\right) \text { for a.e. } t \in(0, T) \\
\frac{d v_{n}}{d t}(t)+D \Phi_{2, n}\left(v_{n}(t)\right)=F_{2, n}\left(t, u_{n}(t), v_{n}(t)\right) \text { for a.e. } t \in(0, T) \\
\underline{\rho}_{1, n} \leq u_{n}^{0}=u_{n}(0) \leq \bar{\rho}_{1, n}, \underline{\rho}_{2, n} \leq v_{n}^{0}=v_{n}(0) \leq \bar{\rho}_{2, n}, u_{n}^{0} \in \operatorname{dom}\left(\Phi_{1, n}\right), v_{n}^{0} \in \operatorname{dom}\left(\Phi_{2, n}\right) .
\end{array}\right.
$$

Assume that

$\left(\mathrm{Hs}_{1}\right) \Phi_{i, n} \stackrel{M}{\rightarrow} \Phi_{i}$ and $\sup _{n \in \mathbb{N}}\left\|\phi_{i, n}\right\|_{L_{\mathcal{H}_{N-1}^{2}}(\partial \Omega)}<+\infty ;$

$\left(\mathrm{Hs}_{2}\right) \sup _{n \in \mathbb{N}} \Phi_{1, n}\left(u_{n}^{0}\right)<+\infty$ and $\sup _{n \in \mathbb{N}} \Phi_{2, n}\left(v_{n}^{0}\right)<+\infty ;$

$\left(\mathrm{Hs}_{3}\right) u_{n}^{0} \rightarrow u^{0}$ and $v_{n}^{0} \rightarrow v^{0}$ strongly in $L^{2}(\Omega)$;

$\left(\mathrm{Hs}_{4}\right) g_{i, n}$ and $h_{i, n}$ pointwise converge to $g_{i}$ and $h_{i}$ respectively;

$\left(\mathrm{Hs}_{5}\right) \sup _{n}\left\|r_{i, n}\right\|_{L^{\infty}\left([0, T] \times \mathbb{R}^{N}, \mathbb{R}^{l}\right)}<+\infty$ and $r_{i, n} \rightarrow r_{i}$ in $L^{2}\left(0, T, L^{2}\left(\Omega, \mathbb{R}^{l}\right)\right)$ where $r_{i} \in L^{\infty}\left([0, T] \times \mathbb{R}^{N}, \mathbb{R}^{l}\right)$;

$\left(\mathrm{Hs}_{6}\right)$ For all $t \in[0, T], \sup _{n}\left\|q_{i, n}(t, \cdot)\right\|_{L^{2}(\Omega)}<+\infty$ and $q_{i, n} \rightarrow q_{i}$ in $L^{2}\left(0, T, L^{2}(\Omega)\right)$.

Then $\left(u_{n}, v_{n}\right)$ uniformly converges in $C\left([0, T], L^{2}(\Omega)\right) \times C\left([0, T], L^{2}(\Omega)\right)$ to the unique solution $(u, v)$ of the system

$$
(\mathcal{S})\left\{\begin{array}{l}
\frac{d u}{d t}(t)+\partial \Phi_{1}(u(t)) \ni F_{1}(t, u(t), v(t)) \text { for a.e. } t \in(0, T) \\
\frac{d v}{d t}(t)+\partial \Phi_{2}(v(t)) \ni F_{2}(t, u(t), v(t)) \text { for a.e. } t \in(0, T) \\
\underline{\rho}_{1} \leq u^{0}=u(0) \leq \bar{\rho}_{1}, \underline{\rho}_{2} \leq v^{0}=v(0) \leq \bar{\rho}_{2}, u^{0} \in \operatorname{dom}\left(\Phi_{1}\right), v^{0} \in \operatorname{dom}\left(\Phi_{2}\right) .
\end{array}\right.
$$

The reaction functionals $F_{i}:[0,+\infty) \times L^{2}(\Omega) \times L^{2}(\Omega) \rightarrow \mathbb{R}^{\Omega}, i=1,2$, are defined for all $t \in[0, T]$, all $(U, V) \in L^{2}(\Omega) \times L^{2}(\Omega)$ and for a.e. $x \in \Omega$, by

$$
\begin{aligned}
& F_{i}(t, U, V)(x)=f_{i}(t, x, U(x), V(x)), \\
& f_{1}\left(t, x, \zeta, \zeta^{\prime}\right)=r_{1}(t, x) \odot h_{1}\left(\zeta^{\prime}\right) \cdot g_{1}(\zeta)+q_{1}(t, x), \\
& f_{2}\left(t, x, \zeta, \zeta^{\prime}\right)=r_{2}(t, x) \odot h_{2}(\zeta) \cdot g_{2}\left(\zeta^{\prime}\right)+q_{2}(t, x) .
\end{aligned}
$$

Moreover $\underline{\rho}_{1} \leq u \leq \bar{\rho}_{1}, \underline{\rho}_{2} \leq v \leq \bar{\rho}_{2}$, and $\left(\frac{d u_{n}}{d t}, \frac{d u_{n}}{d t}\right) \rightarrow\left(\frac{d u}{d t}, \frac{d v}{d t}\right)$ weakly in $L^{2}\left(0, T, L^{2}(\Omega)\right) \times$ $L^{2}\left(0, T, L^{\overline{2}}(\Omega)\right)$.

Furthermore, if $\left(\Phi_{1, n}\left(u_{n}^{0}\right), \Phi_{1, n}\left(v_{n}^{0}\right)\right) \rightarrow\left(\Phi\left(u^{0}\right), \Phi\left(v^{0}\right)\right), r_{i, n} \rightarrow r_{i}$ strongly in $L^{2}\left(0, T, L^{2}\left(\Omega, \mathbb{R}^{l}\right)\right)$, and $q_{i, n} \rightarrow q_{i}$ strongly in $L^{2}\left(0, T, L^{2}(\Omega)\right)$, then $\left(\frac{d u_{n}}{d t}, \frac{d u_{n}}{d t}\right) \rightarrow\left(\frac{d u}{d t}, \frac{d v}{d t}\right)$ strongly in $L^{2}\left(0, T, L^{2}(\Omega)\right) \times$ $L^{2}\left(0, T, L^{2}(\Omega)\right)$.

Proof. Since $\operatorname{dom}\left(\Phi_{i, n}\right) \subset \overline{\operatorname{dom}\left(D \Phi_{i, n}\right)}$, we have $\left(u_{n}^{0}, v_{n}^{0}\right) \in\left(\overline{\operatorname{dom}\left(D \Phi_{1, n}\right)}, \overline{\operatorname{dom}\left(D \Phi_{2, n}\right)}\right)$, so that, according to Theorem 2.1, $\left(\mathcal{P}_{n}\right)$ admits a unique solution $\left(u_{n}, v_{n}\right)$ which satisfies $\left(S_{2}\right)-\left(S_{4}\right)$ of Theorem 2.1. We follow the strategy of the proof of [1, Theorem 4.1].

Step 1. We establish

$$
\begin{aligned}
& \underline{\rho}_{1} \leq u_{n} \leq \bar{\rho}_{1} \text { and } \underline{\rho}_{2} \leq v_{n} \leq \bar{\rho}_{2} ; \\
& \bar{g}_{i}:=\sup _{(\zeta, n) \in\left[\underline{\rho}_{i}, \bar{\rho}_{i}\right] \times \mathbb{N}}\left|g_{i, n}(\zeta)\right|<+\infty, \bar{h}_{i}:=\sup _{(\zeta, n) \in\left[\underline{\rho}_{i}, \bar{\rho}_{i}\right] \times \mathbb{N}}\left|h_{i, n}(\zeta)\right|<+\infty ; \\
& \sup _{n \in \mathbb{N}}\left\|\frac{d u_{n}}{d t}\right\|_{L^{2}(0, T, X)}<+\infty \text { and } \sup _{n \in \mathbb{N}}\left\|\frac{d v_{n}}{d t}\right\|_{L^{2}(0, T, X)}<+\infty .
\end{aligned}
$$


From Theorem 2.1 the solution $\left(u_{n}, v_{n}\right)$ of $\left(\mathcal{S}_{n}\right)$ satisfies $\underline{y}_{1, n}(T) \leq u_{n} \leq \bar{y}_{1, n}(T)$ and $\underline{y}_{2, n}(T) \leq v_{n} \leq$ $\bar{y}_{2, n}(T)$, so that inequalities (3.6) follow directly from (3.4). We deduce (3.7) from (3.2), hypothesis $\left(\mathrm{Hs}_{4}\right)$ and estimate $\left|g_{i, n}(\zeta)\right| \leq\left|g_{i, n}(0)\right|+L_{\left[\underline{\rho}_{i}, \bar{\rho}_{i}\right]}|\zeta|$; idem for $h_{i, n}$.

Let us establish (3.8). In what follows the letter $C$ denotes various constants which can vary from line to line. By using the structure of the TCCP-structured reaction functional $F_{n}$, and from (3.7) and hypothesis $\left(\mathrm{Hs}_{5}\right)$, we easily infer that

$$
\begin{aligned}
\left\|F_{i, n}\left(t, u_{n}(t), v_{n}(t)\right)\right\|_{X}^{2} & \leq 2 \mathcal{L}_{N}(\Omega)\left\|r_{i, n}\right\|_{\infty}^{2} \bar{g}_{i}^{2} \bar{h}_{i}^{2}+2\left\|q_{i, n}(t, \cdot)\right\|_{X}^{2} \\
& \leq C\left(1+\left\|q_{i, n}(t, \cdot)\right\|_{X}^{2}\right) .
\end{aligned}
$$

Thus, according to $\left(\mathrm{Hs}_{6}\right)$, we deduce

$$
\sup _{n} \int_{0}^{T}\left\|F_{i, n}\left(t, u_{n}(t), v_{n}(t)\right)\right\|_{X}^{2} d t<+\infty .
$$

On the other hand, from $\left(\mathcal{S}_{n}\right)$ we infer that for a.e. $t \in(0, T)$,

$$
\begin{aligned}
& \left\|\frac{d u_{n}}{d t}(t)\right\|_{X}^{2}+\left\langle D \Phi_{1, n}\left(u_{n}(t)\right), \frac{d u_{n}}{d t}(t)\right\rangle=\left\langle F_{1, n}\left(t, u_{n}(t), v_{n}(t)\right), \frac{d u_{n}}{d t}(t)\right\rangle \\
& \left\|\frac{d v_{n}}{d t}(t)\right\|_{X}^{2}+\left\langle D \Phi_{2, n}\left(u_{n}(t)\right), \frac{d v_{n}}{d t}(t)\right\rangle=\left\langle F_{2, n}\left(t, u_{n}(t), v_{n}(t)\right), \frac{d v_{n}}{d t}(t)\right\rangle .
\end{aligned}
$$

By integrating over $(0, T)$, we obtain

$$
\begin{aligned}
& \int_{0}^{T}\left\|\frac{d u_{n}}{d t}(t)\right\|_{X}^{2} d t+\int_{0}^{T}\left\langle D \Phi_{1, n}\left(u_{n}(t)\right), \frac{d u_{n}}{d t}(t)\right\rangle d t=\int_{0}^{T}\left\langle F_{1, n}\left(t, u_{n}(t), v_{n}(t)\right), \frac{d u_{n}}{d t}(t)\right\rangle d t, \\
& \int_{0}^{T}\left\|\frac{d v_{n}}{d t}(t)\right\|_{X}^{2} d t+\int_{0}^{T}\left\langle D \Phi_{2, n}\left(v_{n}(t)\right), \frac{d v_{n}}{d t}(t)\right\rangle d t=\int_{0}^{T}\left\langle F_{2, n}\left(t, u_{n}(t), v_{n}(t)\right), \frac{d v_{n}}{d t}(t)\right\rangle d t .
\end{aligned}
$$

Since $\left(u_{n}^{0}, v_{n}^{0}\right) \in\left(\operatorname{dom}\left(\Phi_{1, n}\right), \operatorname{dom}\left(\Phi_{2, n}\right)\right)$, we deduce that $\left(\frac{d u_{n}}{d t}, \frac{d v_{n}}{d t}\right)$ belongs to $L^{2}(0, T, X) \times L^{2}(0, T, X)$ and $t \mapsto \Phi_{1, n}\left(u_{n}(t)\right), t \mapsto \Phi_{2, n}(v(t))$ are absolutely continuous (see [5, Theorem 3.6]). Therefore for a.e. $t \in(0, T), \frac{d}{d t} \Phi_{1, n}\left(u_{n}(t)\right)=\left\langle D \Phi_{1, n}\left(u_{n}(t)\right), \frac{d u_{n}}{d t}(t)\right\rangle$, and $\frac{d}{d t} \Phi_{2, n}\left(v_{n}(t)\right)=\left\langle D \Phi_{2, n}\left(v_{n}(t)\right), \frac{d v_{n}}{d t}(t)\right\rangle$ (see [4, Proposition 17.2.5]). From the first equality in (3.11) we have

$$
\begin{aligned}
\int_{0}^{T}\left\|\frac{d u_{n}}{d t}(t)\right\|_{X}^{2} d t & =-\Phi_{1, n}\left(u_{n}(T)\right)+\Phi_{1, n}\left(u_{n}^{0}\right)+\int_{0}^{T}\left\langle F_{1, n}\left(t, u_{n}(t), v_{n}(t)\right), \frac{d u_{n}}{d t}(t)\right\rangle d t \\
& \leq-\inf _{w \in L^{2}(\Omega)} \Phi_{1, n}(w)+\sup _{n} \Phi_{1, n}\left(u_{n}^{0}\right) \\
& +\left(\int_{0}^{T}\left\|F_{1, n}\left(t, u_{n}(t), v_{n}(t)\right)\right\|_{X}^{2}\right)^{\frac{1}{2}}\left(\int_{0}^{T}\left\|\frac{d u_{n}}{d t}(t)\right\|_{X}^{2}\right)^{\frac{1}{2}}
\end{aligned}
$$

where

$$
\inf _{v \in L^{2}(\Omega)} \Phi_{1, n}(w) \geq-\frac{C_{\text {trace }}}{2 \nu}\left\|\phi_{1, n}\right\|_{L_{\mathcal{H}_{N-1}}^{2}(\partial \Omega)}^{2} .
$$

From $\left(\mathrm{Hs}_{1}\right),\left(\mathrm{Hs}_{2}\right)$, and 3.10$), 3.12$ yields that there exists a constant $C \geq 0$ such that

$$
\int_{0}^{T}\left\|\frac{d u_{n}}{d t}(t)\right\|_{X}^{2} d t \leq C\left(1+\left(\int_{0}^{T}\left\|\frac{d u_{n}}{d t}(t)\right\|_{X}^{2} d t\right)^{\frac{1}{2}}\right) .
$$

Reasoning similarly with the second equality in 3.11, we obtain

$$
\int_{0}^{T}\left\|\frac{d v_{n}}{d t}(t)\right\|_{X}^{2} d t \leq C\left(1+\left(\int_{0}^{T}\left\|\frac{d v_{n}}{d t}(t)\right\|_{X}^{2} d t\right)^{\frac{1}{2}}\right),
$$

from which we deduce 3.8 . 
Step 2. We prove that there exist $(u, v) \in C([0, T], X) \times C([0, T], X)$, and a subsequence of $\left(\left(u_{n}, v_{n}\right)\right)_{n \in \mathbb{N}}$ not relabeled, satisfying $\left(u_{n}, v_{n}\right) \rightarrow(u, v)$ in $C([0, T], X) \times C([0, T], X)$ equipped with the norm $\|\cdot\|_{C \times C}$.

Basically we apply the Ascoli-Arzela compactness theorem for $\left(u_{n}\right)_{n \in \mathbb{N}}$ and $\left(v_{n}\right)_{n \in \mathbb{N}}$. We reason for $\left(u_{n}\right)_{n \in \mathbb{N}}$, the same reasoning holds for $\left(v_{n}\right)_{n \in \mathbb{N}}$. From $(3.6),\left(u_{n}\right)_{n \in \mathbb{N}}$ is bounded in $C([0, T], X)$. Moreover for $(s, t) \in[0, T]^{2}$ with $s<t$, we have

$$
\left\|u_{n}(t)-u_{n}(s)\right\|_{X} \leq \int_{s}^{t}\left\|\frac{d u_{n}}{d t}(\tau)\right\|_{X} d \tau \leq(t-s)^{\frac{1}{2}}\left\|\frac{d u_{n}}{d t}\right\|_{L^{2}(0, T, X)} \leq(t-s)^{\frac{1}{2}} \sup _{n}\left\|\frac{d u_{n}}{d t}\right\|_{L^{2}(0, T, X)}
$$

which, from (3.8), yields the equicontinuity of the sequence $\left(u_{n}\right)_{n \in \mathbb{N}}$. It remains to establish for each $t \in[0, T]$, the relative compactness in $X$ of the set $E_{t}:=\left\{u_{n}(t): n \in \mathbb{N}\right\}$. For $t=0$ there is nothing to prove because of hypothesis $\left(\mathrm{Hs}_{3}\right)$ on the initial condition. It remains to establish the relative compactness of $E_{t}$ for $\left.\left.t \in\right] 0, T\right]$. In what follows $t$ is fixed in $\left.] 0, T\right]$.

According to Theorem 2.1. $u_{n}$ satisfies $\left(S_{4}\right)$, then possesses a right derivative at $t$ (at $t=T$, this is due to the fact that $u_{n}$ exists in $C([0,+\infty), X)$ so that the right derivative of $u_{n}$ at $t=T$ is nothing but the right derivative of the restriction of $u_{n}$ to $\left.[0, T]\right)$. Moreover

$$
\frac{d u_{n}^{+}}{d t}(t)+D \Phi_{1, n}\left(u_{n}(t)\right)=F_{1, n}\left(t, u_{n}(t), v_{n}(t)\right)
$$

Taking $u_{n}(t)$ as a test function, we infer that

$$
\left\langle\frac{d u_{n}^{+}}{d t}(t), u_{n}(t)\right\rangle+\left\langle D \Phi_{1, n}\left(u_{n}(t)\right), u_{n}(t)\right\rangle=\left\langle F_{1, n}\left(t, u_{n}(t), v_{n}(t)\right), u_{n}(t)\right\rangle,
$$

hence, from the Green formula and the fact that $u_{n}(t) \in \operatorname{dom} D \Phi_{1, n}$,

$$
\begin{aligned}
& \int_{\Omega} D_{\xi} W_{1, n}\left(x, \nabla u_{n}(t)\right) \cdot \nabla u_{n}(t) d x \\
& =\int_{\partial \Omega} D_{\xi} W_{1, n}\left(x, \nabla u_{n}(t)\right) \cdot \mathbf{n} u_{n}(t) d \mathcal{H}_{N-1}-\int_{\Omega} \frac{d u_{n}^{+}}{d t}(t) u_{n}(t) d x+\int_{\Omega} F_{1, n}\left(t, u_{n}(t), v_{n}(t)\right) d x \\
& =\int_{\partial \Omega}\left(\phi_{i, n}-a_{1, n} u_{n}(t)\right) u_{n}(t) d \mathcal{H}_{N-1}-\int_{\Omega} \frac{d u_{n}^{+}}{d t}(t) u_{n}(t) d x+\int_{\Omega} F_{1, n}\left(t, u_{n}(t), v_{n}(t)\right) d x \\
& \leq \int_{\partial \Omega} \phi_{i, n} u_{n}(t) d \mathcal{H}_{N-1}-\int_{\Omega} \frac{d u_{n}^{+}}{d t}(t) u_{n}(t) d x+\int_{\Omega} F_{1, n}\left(t, u_{n}(t), v_{n}(t)\right) d x .
\end{aligned}
$$

Take $0<\nu<\frac{2 \gamma}{C_{\text {trace }}}$ where $\gamma$ is the positive constant of the uniform strong convexity condition $\left(\mathrm{D}_{3, n}\right)$ and $C_{\text {trace }}$ is the constant of continuity of the trace operator. Set $b:=\max \left(\left|\underline{\rho}_{1}\right|,\left|\bar{\rho}_{1}\right|\right)$. From $\left(\mathrm{D}_{3, n}\right)$ (3.6) and (3.9), we deduce that

$$
\begin{aligned}
\gamma \int_{\Omega}\left|\nabla u_{n}(t)\right|^{2} d x & \leq\left\|\phi_{1, n}\right\|_{L_{\mathcal{H}_{N-1}^{2}}(\partial \Omega)}\left\|u_{n}(t)\right\|_{L_{\mathcal{H}_{N-1}}^{2}(\partial \Omega)} \\
& +b \mathcal{L}_{N}(\Omega)^{\frac{1}{2}}\left(\left\|\frac{d u_{n}^{+}}{d t}(t)\right\|_{X}+\left\|F_{1, n}\left(t, u_{n}(t), v_{n}(t)\right)\right\|_{X}\right) \\
& \leq \frac{C_{\text {trace }}}{2 \nu}\left\|\phi_{1, n}\right\|_{L_{\mathcal{H}_{N-1}}^{2}(\partial \Omega)}^{2}+\frac{C_{\text {trace }} \nu}{2}\left\|u_{n}(t)\right\|_{H^{1}(\Omega)}^{2} \\
& +b \mathcal{L}_{N}(\Omega)^{\frac{1}{2}}\left(\left\|\frac{d u_{n}^{+}}{d t}(t)\right\|_{X}+\left\|F_{1, n}\left(t, u_{n}(t), v_{n}(t)\right)\right\|_{X}\right) \\
& \leq \frac{C_{\text {trace }}}{2 \nu}\left\|\phi_{1, n}\right\|_{L_{\mathcal{H}_{N-1}}^{2}(\partial \Omega)}^{2}+\frac{C_{\text {trace }} \nu}{2}\left(\int_{\Omega}\left|\nabla u_{n}(t)\right|^{2} d x+b^{2} \mathcal{L}_{N}(\Omega)\right) \\
& +b \mathcal{L}_{N}(\Omega)^{\frac{1}{2}}\left(\left\|\frac{d u_{n}^{+}}{d t}(t)\right\|_{X}+\left\|F_{1, n}\left(t, u_{n}(t), v_{n}(t)\right)\right\|_{X}\right) .
\end{aligned}
$$


Hence

$$
\begin{aligned}
\left(\gamma-\frac{C_{\text {trace }} \nu}{2}\right) \int_{\Omega}\left|\nabla u_{n}(t)\right|^{2} d x \leq & \frac{C_{\text {trace }}}{2 \nu} \sup _{n}\left\|\phi_{1, n}\right\|_{L_{\mathcal{H}_{N-1}^{2}}}^{2}(\partial \Omega) \\
& +b^{2} \frac{C_{\text {trace }} \nu}{2} \mathcal{L}_{N}(\Omega) \\
& +b \mathcal{L}_{N}(\Omega)^{\frac{1}{2}} \sup _{n \in \mathbb{N}}\left(\left\|\frac{d u_{n}^{+}}{d t}(t)\right\|_{X}+\left\|F_{1, n}\left(t, u_{n}(t), v_{n}(t)\right)\right\|_{X}\right) .
\end{aligned}
$$

From 3.10 , estimates 3.13 and 3.6 yield that $\left(u_{n}(t)\right)_{n \in \mathbb{N}}$ is bounded in $H^{1}(\Omega)$ provided that we establish

$$
\sup _{n}\left\|\frac{d u_{n}^{+}}{d t}(t)\right\|_{X}<+\infty .
$$

Then from the compact embedding $H^{1}(\Omega) \hookrightarrow L^{2}(\Omega)$ we will conclude to the compactness of the set $E_{t}$. Therefore, to end $\boldsymbol{S t e p} 2$, it remains to establish (3.14). This estimate requires the sharp result of Lemma B.1.

Set $G_{n}(t):=F_{1, n}\left(t, u_{n}(t), v_{n}(t)\right)$. In order to apply Lemma B.1. we start by establishing the following estimate on the total variation $\operatorname{Var}\left(G_{n},[0, T]\right)$ of $G_{n}$ in $[0, T]$ :

$$
\operatorname{Var}\left(G_{n},[0, T]\right)=\int_{0}^{T}\left\|\frac{G_{n}}{d t}(t)\right\|_{X} d t \leq C\left(1+\int_{0}^{T}\left\|\frac{d v_{n}}{d t}(t)\right\|_{X} d t+\int_{0}^{T}\left\|\frac{d u_{n}}{d t}(t)\right\|_{X} d t\right)
$$

where $C$ is a nonnegative constant which does not depend on $n$. To shorten the notation, we omit the index $i=1$. According to the structure of $F_{1, n}$, to 3.7$),(3.3)$, and hypothesis $\left(\mathrm{Hs}_{6}\right)$, we have

$$
\begin{aligned}
\left\|G_{n}(t)-G_{n}(s)\right\|_{X} \leq & \bar{g} \bar{h}\left\|r_{n}(t)-r_{n}(s)\right\|_{L^{2}\left(\Omega, \mathbb{R}^{l}\right)}+\left\|q_{n}(t)-q_{n}(s)\right\|_{X} \\
& +\sup _{n \in \mathbb{N}}\left\|r_{n}\right\|_{\infty}\left\|g_{n}\left(u_{n}(t)\right) \cdot h_{n}\left(v_{n}(t)\right)-g_{n}\left(u_{n}(s)\right) \cdot h_{n}\left(v_{n}(s)\right)\right\|_{X} \\
\leq & \bar{g} \bar{h} \int_{s}^{t}\left\|\frac{d r_{n}}{d t}(t, \cdot)\right\|_{L^{2}\left(\Omega, \mathbb{R}^{l}\right)} d t+\int_{s}^{t}\left\|\frac{d q_{n}}{d t}(t, \cdot)\right\|_{X} \\
& +\sup _{n \in \mathbb{N}}\left\|r_{n}\right\|_{\infty}\left\|g_{n}\left(u_{n}(t)\right) \cdot h_{n}\left(v_{n}(t)\right)-g_{n}\left(u_{n}(s)\right) \cdot h_{n}\left(v_{n}(s)\right)\right\|_{X} \cdot
\end{aligned}
$$

On the other hand, from 3.2 and 3.7 , we infer that

$$
\begin{aligned}
& \left\|g_{n}\left(u_{n}(t)\right) \cdot h_{n}\left(v_{n}(t)\right)-g_{n}\left(u_{n}(s)\right) \cdot h_{n}\left(v_{n}(s)\right)\right\|_{X} \\
& \leq \bar{g} L_{\left[\underline{\rho}_{2}, \bar{\rho}_{2}\right]}^{\prime}\left\|v_{n}(t)-v_{n}(s)\right\|_{X}+\bar{h} L_{\left[\underline{\rho}_{1}, \bar{\rho}_{1}\right]}\left\|u_{n}(t)-u_{n}(s)\right\|_{X} \\
& \leq g L_{\left[\underline{\rho}_{2}, \bar{\rho}_{2}\right]}^{\prime} \int_{s}^{t}\left\|\frac{d v_{n}}{d \sigma}(\sigma)\right\|_{X} d \sigma+\bar{h} L_{\left[\underline{\rho}_{1}, \bar{\rho}_{1}\right]} \int_{s}^{t}\left\|\frac{d u_{n}}{d \sigma}(\sigma)\right\|_{X} d \sigma .
\end{aligned}
$$

Estimate 3.15) is then obtained by combining 3.16, 3.17, 3.8, and 3.3.

Hence, applying Lemma B.1, from 3.15, we deduce that

$$
\left\|\frac{d u_{n}^{+}}{d t}(t)\right\|_{X} \leq C+C \int_{0}^{T}\left\|\frac{d v_{n}}{d t}(t)\right\|_{X} d t+\left(C+\frac{1}{t}\right) \int_{0}^{T}\left\|\frac{d u_{n}}{d t}(t)\right\|_{X} d t
$$

and (3.14) follows from (3.8). This completes Step 2 .

Step 3. We assert that $\left(\frac{d u_{n}}{d t}, \frac{d v_{n}}{d t}\right) \rightarrow\left(\frac{d u}{d t}, \frac{d v}{d t}\right)$ weakly in $L^{2}(0, T, X) \times L^{2}(0, T, X)$ for a non relabeled subsequence, and that $\underline{\rho}_{1} \leq u \leq \bar{\rho}_{1}, \underline{\rho}_{2} \leq u \leq \bar{\rho}_{2}$. The first claim is a straightforward consequence of (3.8) and Step 2. The second follows from inequality $\underline{\rho}_{1, n} \leq u_{n} \leq \bar{\rho}_{1, n}, \underline{\rho}_{2, n} \leq v_{n} \leq \bar{\rho}_{2, n}$, 3.4), and $\left(u_{n}, v_{n}\right) \rightarrow(u, v)$ in $C([0, T], X)$.

Step 4. We prove that $(u, v)$ is the unique solution of $(\mathcal{S})$. The proof mimics that of 1 , Theorem 4.1]. We give a sketch of the proof. According to the Fenchel extremality condition (see [4, Proposition 9.5.1]), the fact that $\left(u_{n}, v_{n}\right)$ solves $\left(\mathcal{S}_{n}\right)$, is equivalent to

$$
\begin{aligned}
\int_{0}^{T}\left[\Phi_{1, n}\left(u_{n}(t)\right)+\Phi_{1, n}^{*}\right. & \left.\left(G_{1, n}(t)-\frac{d u_{n}}{d t}(t)\right)\right] d t \\
& +\frac{1}{2}\left(\left\|u_{n}(T)\right\|^{2}-\left\|u_{n}^{0}\right\|^{2}\right)-\int_{0}^{T}\left\langle G_{1, n}(t), u_{n}(t)\right\rangle d t=0
\end{aligned}
$$




$$
\begin{aligned}
\int_{0}^{T}\left[\Phi_{2, n}\left(u_{n}(t)\right)+\Phi_{2, n}^{*}\right. & \left.\left(G_{2, n}(t)-\frac{d v_{n}}{d t}(t)\right)\right] d t \\
& +\frac{1}{2}\left(\left\|v_{n}(T)\right\|^{2}-\left\|v_{n}^{0}\right\|^{2}\right)-\int_{0}^{T}\left\langle G_{2, n}(t), u_{n}(t)\right\rangle d t=0
\end{aligned}
$$

where $G_{i, n}(t)=F_{i, n}\left(t, u_{n}(t), v_{n}(t)\right)$. Observe that the functionals defined in $L^{2}\left(0, T, L^{2}(\Omega)\right)$ by

$$
w \mapsto \int_{0}^{T} \Phi_{i, n}(w(t)) d t, \quad w \mapsto \int_{0}^{T} \Phi_{i, n}^{*}(w(t)) d t
$$

Mosco-converge to

$$
w \mapsto \int_{0}^{T} \Phi_{i}(w(t)) d t, \quad w \mapsto \int_{0}^{T} \Phi_{i}^{*}(w(t)) d t
$$

respectively (refer to [1, Lemma 4.1]). Thus going to the limit in two previous equalities, from Step 2, Step 3, and Lemma 3.1 below, we obtain

$$
\begin{aligned}
& \int_{0}^{T}\left[\Phi_{1}(u(t))+\Phi_{1}^{*}\left(G_{1}(t)\right.\right.\left.\left.-\frac{d u}{d t}(t)\right)\right] d t \\
&+\frac{1}{2}\left(\|u(T)\|^{2}-\left\|u^{0}\right\|^{2}\right)-\int_{0}^{T}\left\langle G_{1}(t), u(t)\right\rangle d t=0, \\
& \int_{0}^{T}\left[\Phi_{2}(u(t))+\Phi_{2}^{*}\left(G_{2}(t)-\frac{d v}{d t}(t)\right)\right] d t \\
&+\frac{1}{2}\left(\|v(T)\|^{2}-\left\|v^{0}\right\|^{2}\right)-\int_{0}^{T}\left\langle G_{2}(t), u(t)\right\rangle d t=0,
\end{aligned}
$$

where $G_{i}(t)=F_{i}(t, u(t), v(t))$. Observe that we applied the Legendre-Fenchel inequality in order to obtain equality above. This proves that $(u, v)$ solves $(\mathcal{S})$.

Lemma 3.1. For $i=1,2$, the functional $G_{i, n}=F_{i, n}\left(\cdot, u_{n}, v_{n}\right)$ weakly converges in $L^{2}(0, T, X)$ to the functional $G_{i}$ defined by $G_{i}(t)=F_{i}(t, u(t), v(t))$ where $F_{1}(t, u(t), v(t))=r_{1}(t) \odot h_{1}(v(t)) \cdot g_{1}(u(t))+$ $q_{1}(t)$, and $F_{2}(t, u(t), v(t))=r_{2}(t) \odot h_{2}(v(t)) \cdot g_{2}(u(t))+q_{2}(t)$.

Proof of Lemma 3.1. We only prove the weak convergence of $G_{1}$ and omit index 1 . The weak convergence of $G_{2}$ is similar. Recall that $G_{n}(t)=H_{n}(t)+q_{n}(t)$ where

$$
H_{n}(t)(x)=r_{n}(t, x) \odot h_{n}\left(v_{n}(t, x)\right) \cdot g_{n}\left(u_{n}(t, x)\right)=r_{n}(t, x) \cdot h_{n}\left(v_{n}(t, x)\right) \odot g_{n}\left(u_{n}(t, x)\right) .
$$

Since $q_{n} \rightarrow q$ in $L^{2}(0, T, X)$, we are reduced to prove that $H_{n} \rightarrow H$ in $L^{2}(0, T, X)$ where

$$
H(t)(x)=r(t, x) \odot h(v(t, x)) \cdot g(u(t, x))=r(t, x) \cdot h(v(t, x)) \odot g(u(t, x)) .
$$

Hence, since $r_{n} \rightarrow r$ in $L^{2}(0, T, X)$, it suffices to establish that

$$
h_{n}\left(v_{n}\right) \odot g_{n}\left(u_{n}\right) \rightarrow h(v) \odot g(u)
$$

strongly in $L^{2}\left(\Omega, X^{l}\right)$, where $X^{l}$ denotes the space $L^{2}\left(\Omega, \mathbb{R}^{l}\right)$. We have $4^{4}$

$$
\begin{aligned}
\| & h_{n}\left(v_{n}(t)\right) \odot g_{n}\left(u_{n}(t)\right)-h(v(t)) \odot g(u(t)) \|_{X^{l}} \\
\leq & \left\|h_{n}\left(v_{n}(t)\right) \odot g_{n}\left(u_{n}(t)\right)-h_{n}\left(v_{n}(t)\right) \odot g(u(t))\right\|_{X^{l}} \\
& +\left\|h_{n}\left(v_{n}(t)\right) \odot g(u(t))-h(v(t)) \odot g(u(t))\right\|_{X^{l}} \\
\leq & \bar{h}\left\|g_{n}\left(u_{n}(t)\right)-g(u(t))\right\|_{X^{l}}+\bar{g}\left\|h_{n}\left(v_{n}(t)\right)-h(v(t))\right\|_{X^{l}} \\
\leq & \bar{h} L_{\left[\underline{\rho}_{1}, \bar{\rho}_{1}\right]}\left\|u_{n}(t)-u(t)\right\|_{X}+\bar{h}\left\|g_{n}(u(t))-g(u(t))\right\|_{X^{l}} \\
& +\bar{g} L_{\left[\underline{\rho}_{2}, \bar{\rho}_{2}\right]}^{\prime}\left\|v_{n}(t)-v(t)\right\|_{X}+\bar{g}\left\|h_{n}(v(t))-h(v(t))\right\|_{X^{l}} .
\end{aligned}
$$

Hence, to prove (3.18), it remains to establish that

$$
\int_{0}^{T}\left\|g_{n}(u(t))-g(u(t))\right\|_{X^{l}}^{2} d t \rightarrow 0, \int_{0}^{T}\left\|h_{n}(v(t))-h(v(t))\right\|_{X^{l}}^{2} d t \rightarrow 0
$$

\footnotetext{
${ }^{4}$ To simplify the notation we write $g(v(t))$ for the function $x \mapsto g(v(t, x))$, idem for $h(v(t)), g_{n}(v(t))$ and $h_{n}(v(t))$.
} 


$$
\begin{aligned}
& \int_{0}^{T}\left\|u_{n}(t)-u(t)\right\|_{X}^{2} d t \rightarrow 0 \\
& \int_{0}^{T}\left\|v_{n}(t)-v(t)\right\|_{X}^{2} d t \rightarrow 0 .
\end{aligned}
$$

The two convergences in 3.19 are a straightforward consequence of hypothesis $\left(\mathrm{Hs}_{4}\right)$ and the Lebesgue dominated convergence theorem. Convergences (3.20) and (3.21) follow from Step 2., this completes the proof of Lemma 3.1 .

Step 4 completes the proof of Theorem 3.1 .

\section{Convergence theorem For Problems COUpling R.D.E. AND N.D.R.E.}

We keep the notation of the previous section and assume that $\Phi_{2, n} \equiv 0$. For obtaining the compactness of $\left(v_{n}\right)_{n \in \mathbb{N}}$ in $C\left([0, T], L^{2}(\Omega)\right)$ (Step $\mathcal{2}$ in the proof of Theorem 3.1), we can no longer invoke the strict convexity of $W_{2, n}$ ensured by $\left(\mathrm{D}_{3, n}\right)$. To overcome the difficulty, we assume additional regularity conditions on the reaction functional and the initial condition for the non diffusive equation. To shorten the notation we denote by $\Phi_{n}$ the functional $\Phi_{1, n}$ and by $W_{n}$ the density $W_{1, n}$. The theorem below provides a convergence result for FitzHugh-Nagumo like models (see Example 2.5).

In the following, we equip the spaces $C^{1}\left(\left[\underline{\rho}_{i}, \bar{\rho}_{i}\right], \mathbb{R}^{l}\right), i=1,2$, with their uniform norms defined by

$$
\||\varphi|\|_{\left[\underline{\rho}_{i}, \bar{\rho}_{i}\right]}:=\sup _{\zeta \in\left[\underline{\rho}_{i}, \bar{\rho}_{i}\right]}|\varphi(\zeta)|+\sup _{\zeta \in\left[\underline{\rho}_{i}, \bar{\rho}_{i}\right]}\left|\frac{d \varphi}{d \zeta}(\zeta)\right| .
$$

The spaces $C^{1}\left(\left[\underline{\rho}_{1}, \bar{\rho}_{1}\right], \mathbb{R}^{l}\right) \times C^{1}\left(\left[\underline{\rho}_{2}, \bar{\rho}_{2}\right], \mathbb{R}^{l}\right)$ and $C^{1}\left(\left[\underline{\rho}_{2}, \bar{\rho}_{2}\right], \mathbb{R}^{l}\right) \times C^{1}\left(\left[\underline{\rho}_{1}, \bar{\rho}_{1}\right], \mathbb{R}^{l}\right)$ are endowed with their product norm.

Theorem 4.1. Assume that the sequence of densities $\left(W_{n}\right)_{n \in \mathbb{N}}$ satisfies conditions $\left(\mathrm{D}_{1, n}\right),\left(\mathrm{D}_{2, n}\right)$, $\left(\mathrm{D}_{3, n}\right)$, and that the sequence of TCCP-structured reaction functionals $\left(F_{1, n}, F_{2, n}\right)_{n \in \mathbb{N}}$ satisfies conditions (3.2), (3.3), (3.4), and (3.5). Assume furthermore that $g_{2, n}$ and $h_{2, n}$ belong to $C_{\text {loc }}^{1}\left(\mathbb{R}, \mathbb{R}^{l}\right)$, and that $r_{2, n}$ and $q_{2, n}$ do not depend on the spatial variable. Let $\left(u_{n}, v_{n}\right)$ be the unique solution of the system

$$
\left(\mathcal{S}_{n}\right)\left\{\begin{array}{l}
\frac{d u_{n}}{d t}(t)+D \Phi_{n}\left(u_{n}(t)\right)=F_{1, n}\left(t, u_{n}(t), v_{n}(t)\right) \text { for a.e. } t \in(0, T) \\
\frac{d v_{n}}{d t}(t)=F_{2, n}\left(t, u_{n}(t), v_{n}(t)\right) \text { for a.e. } t \in(0, T) \\
\underline{\rho}_{1, n} \leq u_{n}^{0}=u_{n}(0) \leq \bar{\rho}_{1, n}, \underline{\rho}_{2, n} \leq v_{n}^{0}=v_{n}(0) \leq \bar{\rho}_{2, n}, u_{n}^{0} \in \operatorname{dom}\left(\Phi_{n}\right), v_{n}^{0} \in H^{1}(\Omega) .
\end{array}\right.
$$

Assume $\left(\mathrm{Hs}_{1}\right)$, $\left(\mathrm{Hs}_{2}\right)$, $\left(\mathrm{Hs}_{5}\right)$, $\left(\mathrm{Hs}_{6}\right)$ for $i=1$ and

$\left(\mathrm{Hs}_{3}^{\prime}\right) u_{n}^{0} \rightarrow u^{0}$ strongly in $L^{2}(\Omega)$ and $v_{n}^{0} \rightarrow v^{0}$ weakly in $H^{1}(\Omega)$;

$\left(\mathrm{Hs}_{4}^{\prime}\right)\left(g_{1, n}, h_{1, n}\right)$ pointwise converges to $\left(g_{1}, h_{1}\right)$, and $\left(g_{2, n}, h_{2, n}\right)$ converges to $\left(g_{2}, h_{2}\right)$ in $C^{1}\left(\left[\underline{\rho}_{2}, \bar{\rho}_{2}\right], \mathbb{R}^{l}\right) \times$

$$
C^{1}\left(\left[\underline{\rho}_{1}, \bar{\rho}_{1}\right], \mathbb{R}^{l}\right) .
$$

Then the solution $\left(u_{n}, v_{n}\right)$ uniformly converges in $C\left([0, T], L^{2}(\Omega)\right) \times C\left([0, T], L^{2}(\Omega)\right)$ to the unique solution $(u, v)$ of the system

$$
(\mathcal{S})\left\{\begin{array}{l}
\frac{d u}{d t}(t)+\partial \Phi(u(t)) \ni F_{1}(t, u(t), v(t)) \text { for a.e. } t \in(0, T) \\
\frac{d v}{d t}(t)=F_{2}(t, u(t), v(t)) \text { for a.e. } t \in(0, T) \\
\underline{\rho}_{1} \leq u^{0}=u(0) \leq \bar{\rho}_{1}, \underline{\rho}_{2} \leq v^{0}=v(0) \leq \bar{\rho}_{2}, u^{0} \in \operatorname{dom}(\Phi), v_{0} \in H^{1}(\Omega) .
\end{array}\right.
$$

The reaction functionals $F_{i}:[0,+\infty) \times L^{2}(\Omega) \times L^{2}(\Omega) \rightarrow \mathbb{R}^{\Omega}, i=1,2$, are defined for all $t \in[0, T]$, all $(U, V) \in L^{2}(\Omega) \times L^{2}(\Omega)$ and for a.e. $x \in \Omega$, by 


$$
\begin{aligned}
& F_{i}(t, U, V)(x)=f_{i}(t, x, U(x), V(x)), \\
& f_{1}\left(t, x, \zeta, \zeta^{\prime}\right)=r_{1}(t, x) \odot h_{1}\left(\zeta^{\prime}\right) \cdot g_{1}(\zeta)+q_{1}(t, x), \\
& f_{2}\left(t, \zeta, \zeta^{\prime}\right)=r_{2}(t) \odot h_{2}(\zeta) \cdot g_{2}\left(\zeta^{\prime}\right)+q_{2}(t) .
\end{aligned}
$$

Moreover $\underline{\rho}_{1} \leq u \leq \bar{\rho}_{1}, \underline{\rho}_{2} \leq v \leq \bar{\rho}_{2}$, and $\left(\frac{d u_{n}}{d t}, \frac{d u_{n}}{d t}\right) \rightarrow\left(\frac{d u}{d t}, \frac{d v}{d t}\right)$ weakly in $L^{2}\left(0, T, L^{2}(\Omega)\right) \times$ $L^{2}\left(0, T, L^{2}(\Omega)\right)$.

Furthermore, if $\Phi_{n}\left(u_{n}^{0}\right) \rightarrow \Phi\left(u^{0}\right), r_{i, n} \rightarrow r_{i}$ strongly in $L^{2}\left(0, T, L^{2}\left(\Omega, \mathbb{R}^{l}\right)\right)$, and $q_{i, n} \rightarrow q_{i}$ strongly in $L^{2}\left(0, T, L^{2}(\Omega)\right)$, then $\left(\frac{d u_{n}}{d t}, \frac{d u_{n}}{d t}\right) \rightarrow\left(\frac{d u}{d t}, \frac{d v}{d t}\right)$ strongly in $L^{2}\left(0, T, L^{2}(\Omega)\right) \times L^{2}\left(0, T, L^{2}(\Omega)\right)$.

Proof. The arguments of the proof of Theorem 3.1 remain valid, except those of Step 2. Therefore, we only have to modify the proof of Step 2. Because of the non strict convexity of $\Phi_{2, n} \equiv 0$, the proof of the relative compactness of $F_{t}=\left\{v_{n}(t): n \in \mathbb{N}\right\}$ in $L^{2}(\Omega)$ for $\left.\left.t \in\right] 0, T\right]$ cannot be obtained by following the same arguments. The proof of the relative compactness of $E_{t}=\left\{u_{n}(t): n \in \mathbb{N}\right\}$ in $L^{2}(\Omega)$ for $t \in(0, T]$ remains the same. We are going to estimate $\sup _{n \in \mathbb{N}}\left\|\nabla v_{n}(t)\right\|_{L^{2}(\Omega)}$ by using Grönwall's lemma, and will conclude to the compactness of $F_{t}$, according to the compact embedding $H^{1}(\Omega) \hookrightarrow L^{2}(\Omega)$. Take the distributional derivative of $F_{2}$ with respect to the space variable. We obtain

$$
\begin{aligned}
\nabla F_{2}\left(t, u_{n}(t), v_{n}(t)\right)= & {\left[r_{2, n}(t) \odot \frac{d h_{2, n}}{d \zeta}\left(u_{n}(t)\right) \cdot g_{2, n}\left(v_{n}(t)\right)\right] \nabla u_{n}(t) } \\
& +\left[r_{2, n}(t) \odot h_{2, n}\left(u_{n}(t)\right) \cdot \frac{d g_{2, n}}{d \zeta^{\prime}}\left(v_{n}(t)\right)\right] \nabla v_{n}(t) .
\end{aligned}
$$

In the following, we set for all $t \in[0, T]$ and for a.e. $x \in \Omega$ :

$$
\begin{aligned}
& r_{2, n}(t) \odot \frac{d h_{2, n}}{d \zeta}\left(u_{n}(t, x)\right) \cdot g_{2, n}\left(v_{n}(t, x)\right):=A_{n}(t, x), \\
& r_{2, n}(t) \odot h_{2, n}\left(u_{n}(t, x)\right) \cdot \frac{d g_{2, n}}{d \zeta^{\prime}}\left(v_{n}(t, x)\right):=B_{n}(t, x) .
\end{aligned}
$$

From $(3.6),\left(\mathrm{Hs}_{4}^{\prime}\right)$ and $\left(\mathrm{Hs}_{5}\right)$, we deduce that

$$
\sup _{(t, x, n) \in[0, T] \times \Omega \times \mathbb{N}}\left|A_{n}(t, x)\right|:=\bar{A}<+\infty
$$

and

$$
\sup _{(t, x, n) \in[0, T] \times \Omega \times \mathbb{N}}\left|B_{n}(t, x)\right|:=\bar{B}<+\infty .
$$

Set $V_{n}(t):=\nabla v_{n}(t)$. Take the distributional derivative with respect to the space variable of each term of the second equation of $\left(\mathcal{S}_{n}\right)$. From the previous calculation, we infer that $V_{n}$ solves the Cauchy problem

$$
\left\{\begin{array}{l}
\frac{d V_{n}}{d t}(t)=A_{n}(t) \nabla u_{n}(t)+B_{n}(t) V_{n}(t) \text { for a.e. } t \in(0, T), \\
V_{n}(0)=\nabla v_{n}^{0}
\end{array}\right.
$$

and belongs to $C\left([0, T], X^{N}\right)$ where $X^{N}:=L^{2}\left(\Omega, \mathbb{R}^{N}\right)$ (see for instance [1, Theorem 2.3] with $F(t, V)=$ $A_{n}(t) \nabla u_{n}(t)+B_{n}(t) V$, and $X^{N}$ substitute for $\left.X\right)$. Hence, for all $t \in(0, T]$,

$$
V_{n}(t)=V_{n}(0)+\int_{0}^{t}\left(A_{n}(s) \nabla u_{n}(s)+B_{n}(t) V_{n}(s)\right) d s,
$$

from which we deduce

$$
\left\|V_{n}(t)\right\|_{X^{N}} \leq\left\|\nabla v_{n}^{0}\right\|_{X^{N}}+\bar{A} \int_{0}^{t}\left\|\nabla u_{n}(s)\right\|_{X^{N}} d s+\bar{B} \int_{0}^{t}\left\|V_{n}(s)\right\|_{X^{N}} d s .
$$

According to Grönwall's lemma, we infer that for all $t \in(0, T]$ (note that $s \mapsto\left\|V_{n}(s)\right\|_{X^{N}}$ is continuous in $[0, T])$

$$
\left\|V_{n}(t)\right\|_{X^{N}} \leq\left(\left\|\nabla v_{n}^{0}\right\|_{X^{N}}+\bar{A} \int_{0}^{T}\left\|\nabla u_{n}(s)\right\|_{X^{N}} d s\right) \exp (\bar{B} T) .
$$

From $\left(\mathrm{Hs}_{3}^{\prime}\right)$ and the fact that $\left(u_{n}(t)\right)_{n \in \mathbb{N}}$ is bounded in $H^{1}(\Omega)$ for all $t \in(0, T]$ (see Step 2 in the proof of Theorem 3.1, we infer from the estimate above, that $\left(\nabla v_{n}(t)\right)_{n \in \mathbb{N}}$ is bounded in $X^{N}$. Therefore $u_{n}(t)$ is bounded in $H^{1}(\Omega)$ for all $t \in[0, T]$, which completes the proof. 
In the specific case of a coupling between two non diffusive reaction equations, we have the following convergence of $\left(\mathcal{S}_{n}\right)$ to $(\mathcal{S})$. The proof is an easy adaptation of the proof above.

Theorem 4.2 (Convergence theorem for problems coupling two n.d.r.e.). Assume that for $i=1,2$, the sequence of TCCP-structured reaction functionals $\left(F_{i, n}\right)_{n \in \mathbb{N}}$ satisfies conditions (3.2), (3.3), (3.4), that $g_{i}$ and $h_{i}$ belong to $C_{l o c}^{1}\left(\mathbb{R}, \mathbb{R}^{l}\right)$, and that $r_{i, n}$ and $q_{i, n}$ do not depend on the spatial variable. Let $\left(u_{n}, v_{n}\right)$ be the unique solution of the system of n.d.r.e.

$$
\left(\mathcal{S}_{n}\right)\left\{\begin{array}{l}
\frac{d u_{n}}{d t}(t)=F_{1, n}\left(t, u_{n}(t), v_{n}(t)\right) \text { for a.e. } t \in(0, T) \\
\frac{d v_{n}}{d t}(t)=F_{2, n}\left(t, u_{n}(t), v_{n}(t)\right) \text { for a.e. } t \in(0, T) \\
\underline{\rho}_{1, n} \leq u_{n}^{0}=u_{n}(0) \leq \bar{\rho}_{1, n}, \underline{\rho}_{2, n} \leq v_{n}^{0}=v_{n}(0) \leq \bar{\rho}_{2, n}, u_{n}^{0} \in H^{1}(\Omega), v_{n}^{0} \in H^{1}(\Omega) .
\end{array}\right.
$$

Assume $\left(\mathrm{Hs}_{5}\right),\left(\mathrm{Hs}_{6}\right)$, and

$\left(\mathrm{Hs}_{3}{ }_{3}\right) u_{n}^{0} \rightarrow u^{0}$ and $v_{n}^{0} \rightarrow v^{0}$ weakly in $H^{1}(\Omega)$;

$\left(\mathrm{Hs}_{4}\right)$ for $i=1,2,\left(g_{i, n}, h_{i, n}\right)$ converges to $\left(g_{i}, h_{i}\right)$ in $C^{1}\left(\left[\underline{\rho}_{1}, \bar{\rho}_{1}\right], \mathbb{R}^{l}\right) \times C^{1}\left(\left[\underline{\rho}_{2}, \bar{\rho}_{2}\right], \mathbb{R}^{l}\right)(i=1)$, and

$$
C^{1}\left(\left[\underline{\rho}_{2}, \bar{\rho}_{2}\right], \mathbb{R}^{l}\right) \times C^{1}\left(\left[\underline{\rho}_{1}, \bar{\rho}_{1}\right], \mathbb{R}^{l}\right)(i=2) .
$$

Then the solution $\left(u_{n}, v_{n}\right)$ uniformly converges in $C\left([0, T], L^{2}(\Omega)\right) \times C\left([0, T], L^{2}(\Omega)\right)$ to the unique solution $(u, v)$ of the system

$$
(\mathcal{S})\left\{\begin{array}{l}
\frac{d u}{d t}(t)=F_{1}(t, u(t), v(t)) \text { for a.e. } t \in(0, T) \\
\frac{d v}{d t}(t)=F_{2}(t, u(t), v(t)) \text { for a.e. } t \in(0, T) \\
\underline{\rho}_{1} \leq u^{0}=u(0) \leq \bar{\rho}_{1}, \underline{\rho}_{2} \leq v^{0}=v(0) \leq \bar{\rho}_{2}, u^{0} \in H^{1}(\Omega), v_{0} \in H^{1}(\Omega) .
\end{array}\right.
$$

The reaction functionals $F_{i}:[0,+\infty) \times L^{2}(\Omega) \times L^{2}(\Omega) \rightarrow \mathbb{R}^{\Omega}, i=1,2$, are defined for all $t \in[0, T]$, all $(U, V) \in L^{2}(\Omega) \times L^{2}(\Omega)$ and for a.e. $x \in \Omega$, by

$$
\begin{aligned}
& F_{i}(t, U, V)(x)=f_{i}(t, x, U(x), V(x)), \\
& f_{1}\left(t, \zeta, \zeta^{\prime}\right)=r_{1}(t) \odot h_{1}\left(\zeta^{\prime}\right) \cdot g_{1}(\zeta)+q_{1}(x), \\
& f_{2}\left(t, \zeta, \zeta^{\prime}\right)=r_{2}(t) \odot h_{2}(\zeta) \cdot g_{2}\left(\zeta^{\prime}\right)+q_{2}(t) .
\end{aligned}
$$

Moreover $\underline{\rho}_{1} \leq u \leq \bar{\rho}_{1}, \underline{\rho}_{2} \leq v \leq \bar{\rho}_{2}$, and $\left(\frac{d u_{n}}{d t}, \frac{d u_{n}}{d t}\right) \rightarrow\left(\frac{d u}{d t}, \frac{d v}{d t}\right)$ weakly in $L^{2}\left(0, T, L^{2}(\Omega)\right) \times$ $L^{2}\left(0, T, L^{\frac{1}{2}}(\Omega)\right)$.

Furthermore, if $r_{i, n} \rightarrow r_{i}$ strongly in $L^{2}\left(0, T, \mathbb{R}^{l}\right)$, and $q_{i, n} \rightarrow q_{i}$ strongly in $L^{2}(0, T)$, then $\left(\frac{d u_{n}}{d t}, \frac{d u_{n}}{d t}\right) \rightarrow$ $\left(\frac{d u}{d t}, \frac{d v}{d t}\right)$ strongly in $L^{2}\left(0, T, L^{2}(\Omega)\right) \times L^{2}\left(0, T, L^{2}(\Omega)\right)$.

Sketch of the proof. We follow again the proof of Theorem 4.1 and we only have to modify the proof of Step 2. The proof of the relative compactness of $E_{t}=\left\{u_{n}(t): n \in \mathbb{N}\right\}$ and $F_{t}=\left\{v_{n}(t): n \in \mathbb{N}\right\}$ in $L^{2}(\Omega)$ for $t \in(0, T]$, is established following the strategy of the proof of Theorem 4.1. Set $U_{n}(t):=$ $\nabla u_{n}(t)$ and $V_{n}(t):=\nabla v_{n}(t)$. We are reduced to prove that

$$
\sup _{n \in \mathbb{N}}\left\|U_{n}(t)\right\|_{X^{N}}<+\infty \text { and } \sup _{n \in \mathbb{N}}\left\|V_{n}(t)\right\|_{X^{N}}<+\infty .
$$

Take the distributional derivative with respect to the space variable of each term of two equations of $\left(\mathcal{S}_{n}\right)$. We infer that $\left(U_{n}, V_{n}\right)$ solves the Cauchy system

$$
\left\{\begin{array}{l}
\frac{d U_{n}}{d t}(t)=C_{n}(t) V_{n}(t)+D_{n}(t) U_{n}(t) \text { for a.e. } t \in(0, T), \\
\frac{d V_{n}}{d t}(t)=A_{n}(t) U_{n}(t)+B_{n}(t) V_{n}(t) \text { for a.e. } t \in(0, T), \\
U_{n}(0)=\nabla u_{n}^{0}, V_{n}(0)=\nabla v_{n}^{0},
\end{array}\right.
$$


in $C\left([0, T], X^{N}\right) \times C\left([0, T], X^{N}\right)$, where $C_{n}$ and $D_{n}$ are defined as $A_{n}$ and $B_{n}$ in the proof of Theorem 4.1, with an obvious adaptation. From 3.6$),\left(\mathrm{Hs}_{4}\right)$ and $\left(\mathrm{Hs}_{5}\right)$ we deduce that

and

$$
\sup _{(t, x, n) \in[0, T] \times \Omega \times \mathbb{N}}\left|C_{n}(t, x)\right|:=\bar{C}<+\infty
$$

Hence, for all $t \in(0, T]$,

$$
\sup _{(t, x, n) \in[0, T] \times \Omega \times \mathbb{N}}\left|D_{n}(t, x)\right|:=\bar{D}<+\infty .
$$

$$
\left\{\begin{array}{l}
U_{n}(t)=U_{n}(0)+\int_{0}^{t}\left(C_{n}(s) V_{n}(s)+D_{n}(t) U_{n}(s)\right) d s \\
V_{n}(t)=V_{n}(0)+\int_{0}^{t}\left(A_{n}(s) U_{n}(s)+B_{n}(t) V_{n}(s)\right) d s
\end{array}\right.
$$

from which we deduce

$$
\left\{\begin{array}{l}
\left\|U_{n}(t)\right\|_{X^{N}} \leq\left\|\nabla u_{n}^{0}\right\|_{X^{N}}+\bar{C} \int_{0}^{t}\left\|V_{n}(s)\right\|_{X^{N}} d s+\bar{D} \int_{0}^{t}\left\|U_{n}(s)\right\|_{X^{N}} d s, \\
\left\|V_{n}(t)\right\|_{X^{N}} \leq\left\|\nabla v_{n}^{0}\right\|_{X^{N}}+\bar{A} \int_{0}^{t}\left\|U_{n}(s)\right\|_{X^{N}} d s+\bar{B} \int_{0}^{t}\left\|V_{n}(s)\right\|_{X^{N}} d s .
\end{array}\right.
$$

From Grönwall's lemma and the first equation, we infer that for all $t \in(0, T]$

so that the second equation gives

$$
\left\|U_{n}(t)\right\|_{X^{N}} \leq\left(\left\|\nabla u_{n}^{0}\right\|_{X^{N}}+\bar{C} \int_{0}^{t}\left\|V_{n}(s)\right\|_{X^{N}} d s\right) \exp (\bar{D} T),
$$

$$
\begin{aligned}
\left\|V_{n}(t)\right\|_{X^{N}} & \leq\left\|\nabla v_{n}^{0}\right\|_{X^{N}}+\bar{A} \int_{0}^{t}\left(\left\|\nabla u_{n}^{0}\right\|_{X^{N}}+\bar{C} \int_{0}^{s}\left\|V_{n}(\sigma)\right\|_{X^{N}} d \sigma\right) \exp (\bar{D} T) d s \\
& +\bar{B} \int_{0}^{t}\left\|V_{n}(s)\right\|_{X^{N}} d s \\
& \leq\left\|\nabla v_{n}^{0}\right\|_{X^{N}}+\bar{A} T \exp (\bar{D} T)\left\|\nabla u_{n}^{0}\right\|_{X^{N}}+(T \exp (\bar{D} T) \overline{A C}+\bar{B}) \int_{0}^{t}\left\|V_{n}(s)\right\|_{X^{N}} d s
\end{aligned}
$$

By applying again Grönwall's lemma, we finally obtain that for all $t \in[0, T]$,

$$
\left\|V_{n}(t)\right\|_{X^{N}} \leq\left(\left\|\nabla v_{n}^{0}\right\|_{X^{N}}+\bar{A} T \exp (\bar{D} T)\left\|\nabla u_{n}^{0}\right\|_{X^{N}}\right) \exp (T(T \exp (\bar{D} T) \overline{A C}+\bar{B})) .
$$

From $\left(\mathrm{Hs}_{1}\right)$ we infer that $\sup _{n \in \mathbb{N}}\left\|V_{n}(t)\right\|_{X^{N}}<+\infty$. Switching the role of $V_{n}$ and $U_{n}$, we obtain that $\sup _{n \in \mathbb{N}}\left\|U_{n}(t)\right\|_{X^{N}}<+\infty$.

\section{Stochastic homogenization of two COMPOnENTS REACTION DifFusion Systems}

We place this section within the framework of stochastic homogenization introduced in 11. In all what follows, $\left(\Sigma, \mathcal{A}, \mathbf{P},\left(T_{z}\right)_{z \in \mathbb{Z}^{N}}\right)$ is a discrete dynamical system, $\mathcal{F}$ denotes the $\sigma$-algebra of invariant sets of $\mathcal{A}$ by the group $\left(T_{z}\right)_{z \in \mathbb{Z}^{N}}$ and, for every $\mathbf{h}$ in the space $L_{\mathbf{P}}(\Sigma)$ of $\mathbf{P}$-integrable functions, $\mathbf{E}^{\mathcal{F}} \mathbf{h}$ denotes the conditional expectation of $\mathbf{h}$ with respect to $\mathcal{F}$ (for the relevant definitions, we refer to 6 ] or [4, Section 12.4] and references therein). We first specify the random diffusion part by recalling some results obtained in [1, Section 5].

5.1. The random diffusion part. Given $\alpha>0$ and $\beta>0$, denote by $\operatorname{Conv}_{\alpha, \beta}$ the class of functions $\mathbf{g}: \mathbb{R}^{N} \times \mathbb{R}^{N} \rightarrow \mathbb{R},(x, \xi) \mapsto \mathbf{g}(x, \xi)$, satisfying conditions $\left(D_{1}\right),\left(D_{2}\right),\left(D_{3}\right)$, and $\left(D_{4}\right)$. We equip Conv $\alpha, \beta$

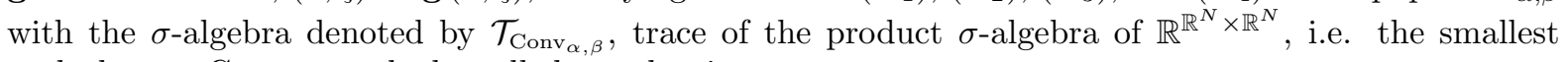
$\sigma$-algebra on $\operatorname{Conv}_{\alpha, \beta}$ such that all the evaluation maps

$$
e_{(x, \xi)}: \mathbf{g} \mapsto \mathbf{g}(x, \xi),(x, \xi) \in \mathbb{R}^{N} \times \mathbb{R}^{N}
$$

are measurable when $\mathbb{R}$ is endowed with its Borel $\sigma$-algebra.

For $i=1,2$, we are given a random convex integrand $W_{i}: \Sigma \times \mathbb{R}^{N} \times \mathbb{R}^{N} \rightarrow \mathbb{R}$, that is to say, a $\left(\mathcal{A} \otimes \mathcal{B}\left(\mathbb{R}^{N}\right) \otimes \mathcal{B}\left(\mathbb{R}^{N}\right), \mathcal{B}(\mathbb{R})\right)$ measurable function such that for every $\omega \in \Sigma$, the function $W_{i}(\omega, ., \cdot)$, 
belongs to the class $\operatorname{Conv}_{\alpha, \beta}$. Since for all $(x, \xi) \in \mathbb{R}^{N} \times \mathbb{R}^{N}, \omega \mapsto W_{i}(\omega, x, \xi)$ is $(\mathcal{A}, \mathcal{B}(\mathbb{R}))$ measurable, the map $\widetilde{W}_{i}: \Sigma \rightarrow \operatorname{Conv}_{\alpha, \beta}, \omega \mapsto W_{i}(\omega, ., \cdot)$, is $\left(\mathcal{A}, \mathcal{T}_{\operatorname{Conv}_{\alpha, \beta}}\right)$ measurable, and we denote by $\widetilde{\mathbf{P}}$ its law, that is $\widetilde{\mathbf{P}}=\widetilde{W} \# \mathbf{P}$.

We assume that $W_{i}$ fulfills the following covariance property with respect to the dynamical system $\left(\Sigma, \mathcal{A}, \mathbf{P},\left(T_{z}\right)_{z \in \mathbb{Z}^{N}}\right)$ : for all $z \in \mathbb{Z}^{N}$

$$
W_{i}\left(T_{z} \omega, x ., \xi\right)=W_{i}(\omega, x+z, \xi) \text { for a.e. } x \in \mathbb{R}^{N} \text {, for all } \xi \in \mathbb{R}^{N} \text {, and for } \mathbf{P} \text { a.e. } \omega \in \Sigma \text {. }
$$

For all $g$ in $\operatorname{Conv}_{\alpha, \beta}$ and all $z \in \mathbb{Z}^{N}$, let us set $\widetilde{T}_{z} g(x, \cdot)=g(x+z, \cdot)$. This defines a group $\left(\widetilde{T}_{z}\right)_{z \in \mathbb{Z}^{N}}$ acting on the class $\operatorname{Conv}_{\alpha, \beta}$, and clearly, for all $z \in \mathbb{Z}^{N}, \widetilde{T}_{z}: \operatorname{Conv}_{\alpha, \beta} \rightarrow \operatorname{Conv}_{\alpha, \beta}$ is $\mathcal{T}_{\operatorname{Conv}_{\alpha, \beta}}$-measurable. Then it is easy to show that the covariance property implies that the law $\widetilde{\mathbf{P}}_{i}$ of $\widetilde{W}_{i}$ is invariant under the group $\left(\widetilde{T}_{z}\right)_{z \in \mathbb{Z}^{N}}$, that is $\widetilde{T}_{z} \# \widetilde{\mathbf{P}}_{i}=\widetilde{\mathbf{P}}_{i}$ for all $z \in \mathbb{Z}^{N}$. Each random function $W_{i}$ is said to be periodic in law.

We write $\varepsilon$ to denote a sequence $\left(\varepsilon_{n}\right)_{n \in \mathbb{N}}$ of positive numbers $\varepsilon_{n}$ going to zero when $n \rightarrow+\infty$, and we briefly write $\varepsilon \rightarrow 0$ instead of $\lim _{n \rightarrow+\infty} \varepsilon_{n}=0$. For $i=1,2$, we consider $\Phi_{i, \varepsilon}: \Sigma \times L^{2}(\Omega) \longrightarrow \mathbb{R}^{+} \cup\{+\infty\}$ defined by

$$
\Phi_{i, \varepsilon}(\omega, u)= \begin{cases}\int_{\Omega} W_{i}\left(\omega, \frac{x}{\varepsilon}, \nabla u\right) d x+\frac{1}{2} \int_{\partial \Omega} a_{i} u^{2} d \mathcal{H}_{N-1}-\int_{\partial \Omega} \phi_{i} u d \mathcal{H}_{N-1} & \text { if } u \in H^{1}(\Omega), \\ +\infty & \text { otherwise }\end{cases}
$$

where $\phi_{i} \in L_{\mathcal{H}_{N-1}}^{2}(\partial \Omega), a_{i} \in L_{\mathcal{H}_{N-1}}^{\infty}(\partial \Omega)$ with $a_{i} \geq 0 \mathcal{H}_{N-1}$ a.e. in $\partial \Omega$, and $a_{i} \geq \sigma_{i}$ on $\Gamma_{i} \subset \partial \Omega$ with $\mathcal{H}_{N-1}\left(\Gamma_{i}\right)>0$ for some $\sigma_{i}>0$. These functionals model random energies concerning various steadystates situations, where the small parameter $\varepsilon$ accounts for the size of small and randomly distributed heterogeneities in the context of a statistically homogeneous media.

Under above hypotheses on $\widetilde{W}_{i}$ with respect to the discrete dynamical system $\left(\Sigma, \mathcal{A}, \mathbf{P},\left(T_{z}\right)_{z \in \mathbb{Z}^{N}}\right)$, using the subadditive ergodic theorem (4 Theorem 12.4.3]), together with [1, Proposition 4.2] we establish that $\mathbf{P}$-almost surely, the sequence of functional $\Phi_{i, \varepsilon}(\omega, \cdot)_{\varepsilon>0}$ Mosco-converges to the integral functional $\Phi_{i}^{\text {hom }}(\omega, \cdot), \Phi_{i}^{\text {hom }}: \Sigma \times L^{2}(\Omega) \longrightarrow \mathbb{R}^{+} \cup\{+\infty\}$ where

$$
\Phi_{i}^{h o m}(\omega, u)= \begin{cases}\int_{\Omega} W_{i}^{h o m}(\omega, \nabla u) d x+\frac{1}{2} \int_{\partial \Omega} a_{i} u^{2} d \mathcal{H}_{N-1}-\int_{\partial \Omega} \phi_{i} u d \mathcal{H}_{N-1} & \text { if } u \in H^{1}(\Omega), \\ +\infty & \text { otherwise. }\end{cases}
$$

The density $W_{i}^{\text {hom }}$ is given, for $\mathbf{P}$ a.s. $\omega \in \Sigma$, and for every $\zeta \in \mathbb{R}^{N}$, by

$$
\begin{aligned}
W_{i}^{h o m}(\omega, \xi) & =\lim _{n \rightarrow+\infty} \inf \left\{\frac{1}{n^{N}} \int_{n Y} W_{i}(\omega, y, \xi+\nabla u(y)) d y: u \in H_{0}^{1}(Y)\right\} \\
& =\inf _{n \in \mathbb{N}^{*}} \mathbf{E}^{\mathcal{F}} \inf \left\{\frac{1}{n^{N}} \int_{n Y} W_{i}(\omega, y, \xi+\nabla u(y)) d y: u \in H_{0}^{1}(Y)\right\},
\end{aligned}
$$

where $Y$ denotes the unit cell $(0,1)^{N}$. If $\left(\Sigma, \mathcal{A}, \mathbf{P},\left(T_{z}\right)_{z \in \mathbb{Z}^{N}}\right)$ is ergodic, then $W_{i}^{h o m}$ is deterministic and given for $\mathbf{P}$ a.s. $\omega \in \Sigma$ by

$$
\begin{aligned}
W_{i}^{h o m}(\xi) & =\lim _{n \rightarrow+\infty} \inf \left\{\frac{1}{n^{N}} \int_{n Y} W_{i}(\omega, y, \xi+\nabla u(y)) d y: u \in H_{0}^{1}(Y)\right\} \\
& =\inf _{n \in \mathbb{N}^{*}} \mathbf{E} \inf \left\{\frac{1}{n^{N}} \int_{n Y} W_{i}(\omega, y, \xi+\nabla u(y)) d y: u \in H_{0}^{1}(Y)\right\} .
\end{aligned}
$$

For a proof we refer the reader to [4, Proposition 12.4.3, Theorem 12.4.7].

For $\mathbf{P}$ a.s. $\omega \in \Sigma$, the subdifferential of $\Phi_{i, \varepsilon}(\omega, \cdot)$ (actually its Gâteaux-derivative) is the operator $A_{i, \varepsilon}(\omega): L^{2}(\Omega) \rightarrow 2^{L^{2}(\Omega)}$ defined for every $\omega \in \Sigma$ by

$\operatorname{dom} A_{i, \varepsilon}(\omega)=\left\{v \in H^{1}(\Omega): \operatorname{div} D_{\xi} W_{i}\left(\omega, \frac{\dot{\bar{\varepsilon}}}{\varepsilon}, \nabla v\right) \in L^{2}(\Omega), a_{i} v+D_{\xi} W_{i}\left(\omega, \frac{\dot{\frac{\varepsilon}{\varepsilon}}}{\varepsilon}, \nabla v\right) \cdot \mathbf{n}=\phi_{i}\right.$ on $\left.\partial \Omega\right\}$

and, for all $v \in \operatorname{dom} A_{i, \varepsilon}(\omega)$,

$$
A_{i, \varepsilon}(\omega) v=-\operatorname{div} D_{\xi} W_{i}(\omega, \dot{\dot{\varepsilon}}, \nabla v)
$$


Similarly the subdifferential of $\Phi_{i}^{\text {hom }}(\omega, \cdot)$ is the multivalued operator $A_{i}^{\text {hom }}(\omega): L^{2}(\Omega) \rightarrow 2^{L^{2}(\Omega)}$ defined for every $\omega \in \Sigma$ by

$$
\operatorname{dom} A_{i}^{h o m}(\omega)=\left\{v \in H^{1}(\Omega): \operatorname{div} D_{\xi} W_{i}^{h o m}(\omega, \nabla v) \in L^{2}(\Omega), a_{i} v+D_{\xi} W_{i}^{h o m}(\omega, \nabla v) \cdot \mathbf{n}=\phi_{i} \text { on } \partial \Omega\right\}
$$

and, for all $v \in \operatorname{dom} A_{i}^{\text {hom }}(\omega)$,

$$
A_{i}^{h o m}(\omega) v=-\operatorname{div} \partial W_{i}^{h o m}(\omega, \nabla v) .
$$

To shorten the notation, we write indifferently $\partial_{\xi} W^{\text {hom }}(\omega, \cdot)$ to denote the subdifferential of $W^{\text {hom }}(\omega, \cdot)$ or any of its elements. When $W_{i}$ is ergodic, then $A_{i}^{\text {hom }}$ is deterministic and

$$
A_{i}^{h o m} v=-\operatorname{div} D_{\xi} W_{i}^{h o m}(\nabla v) .
$$

Recall that from [4, Proposition 17.4.6], $A_{i}^{\text {hom }}(\omega)$ is the $\mathbf{P}$-almost sure graph limit of the operator $A_{i, \varepsilon}(\omega)$. Furthermore, under the following condition on the Fenchel conjugate of $\xi \mapsto W_{i}(\omega, x, \xi)$ :

$\left(\mathrm{D}_{3}{ }_{3}\right)$ there exists $\gamma^{*}>0$ such that $\left\langle\xi_{1}^{*}-\xi_{2}^{*}, \xi^{1}-\xi^{2}\right\rangle \geq \gamma^{*}\left|\xi_{1}-\xi_{2}\right|^{2}$ for $\mathbf{P}$ a.s. $\omega \in \Sigma$, for a.e. $x \in \mathbb{R}^{N}$, for all $\left(\xi_{1}, \xi_{2}\right) \in \mathbb{R}^{N} \times \mathbb{R}^{N}$ and all $\left(\xi_{1}^{*}, \xi_{2}^{*}\right) \in \partial_{\xi} W_{i}^{*}\left(\omega, x, \xi_{1}\right) \times \partial_{\xi} W_{i}^{*}\left(\omega, x, \xi_{2}\right)$,

the density $W_{i}^{\text {hom }}(\omega, \cdot)$ is Gâteaux-differentiable for $\mathbf{P}$ a.s. $\omega \in \Sigma$, and is the pointwise limit of $D_{\xi} W_{i, n}(\omega, \cdot)$ where $W_{i, n}(\omega, \xi)=\inf \left\{\frac{1}{n^{N}} \int_{n Y} W_{i}(\omega, y, \xi+\nabla u(y)) d y: u \in H_{0}^{1}(Y)\right\}$.

5.2. The random reaction parts. We are given a random TCCP-structured reaction functional, i.e. a pair $\left(F_{1}, F_{2}\right)$ with $F_{i}: \Sigma \times[0,+\infty) \times L^{2}(\Omega) \times L^{2}(\Omega) \rightarrow \mathbb{R}^{\Omega}, i=1,2$ defined by $F_{i}(\omega, t, u, v)(x)=$ $f_{i}(\omega, t, x, u(x), v(x))$ where

$$
f_{i}: \Sigma \times[0,+\infty) \times \mathbb{R}^{N} \times \mathbb{R} \times \mathbb{R} \rightarrow \mathbb{R}
$$

is a $\left(\mathcal{A} \otimes \mathcal{B}(\mathbb{R}) \otimes \mathcal{B}\left(\mathbb{R}^{N}\right) \otimes \mathcal{B}(\mathbb{R}) \otimes \mathcal{B}(\mathbb{R}), \mathcal{B}(\mathbb{R})\right)$ measurable function such that for $\mathbf{P}$ a.s. $\omega \in \Sigma$, $\left(f_{1}(\omega, \cdot, \cdot, \cdot, \cdot), f_{2}(\omega, \cdot, \cdot, \cdot, \cdot)\right)$ is a TCSVR-function associated with $\left(r_{i}(\omega, \cdot), g_{i}, h_{i}, q_{i}(\omega, \cdot)\right)$. We assume that $r_{i} \in W^{1,2}\left(0, T, L_{\text {loc }}^{2}\left(\mathbb{R}^{N}, \mathbb{R}^{l}\right)\right), q_{i} \in W^{1,2}\left(0, T, L_{\text {loc }}^{2}\left(\mathbb{R}^{N}\right)\right)$, and for all bounded Borel sets $B$ of $\mathbb{R}^{N}$, the real valued functions

$$
\begin{aligned}
\omega & \mapsto\left\|r_{i}(\omega, t, \cdot)\right\|_{L^{2}\left(B, \mathbb{R}^{l}\right)}^{2} \text { for all } t \in[0, T], \\
\omega & \mapsto\left\|q_{i}(\omega, t, \cdot)\right\|_{L^{2}(B)}^{2} \text { for all } t \in[0, T], \\
\omega & \mapsto \int_{0}^{T}\left\|\frac{d r_{i}}{d t}(\omega, \tau, \cdot)\right\|_{L^{2}\left(B, \mathbb{R}^{l}\right)}^{2} d \tau, \\
\omega & \mapsto \int_{0}^{T}\left\|\frac{d q_{i}}{d t}(\omega, \tau, \cdot)\right\|_{L^{2}(B)}^{2} d \tau
\end{aligned}
$$

belong to $L_{\mathbf{P}}^{1}(\Sigma)$. We also assume that $r_{i}$ and $q_{i}$, satisfy the covariance property with respect to the dynamical system $\left(\Sigma, \mathcal{A}, \mathbf{P},\left(T_{z}\right)_{z \in \mathbb{Z}^{N}}\right)$, i.e. for all $z \in \mathbb{Z}^{N}$, all $t \in[0,+\infty)$, a.e. $x \in \mathbb{R}^{N}$ and $\mathbf{P}$ a.s. $\omega \in \Sigma$,

$$
\begin{aligned}
& r_{i}(\omega, t, x+z)=r_{i}\left(T_{z} \omega, t, x\right), \\
& q_{i}(\omega, t, x+z)=q_{i}\left(T_{z} \omega, t, x\right) .
\end{aligned}
$$

We set $f_{i, \varepsilon}\left(\omega, t, x, \zeta, \zeta^{\prime}\right):=f_{i}\left(\omega, t, \frac{x}{\varepsilon}, \zeta, \zeta^{\prime}\right)$, and define the reaction functional $F_{i, \varepsilon}$ by setting

$$
F_{i, \varepsilon}(\omega, t, u, v)(x)=f_{i}\left(\omega, t, \frac{x}{\varepsilon}, u(x), v(x)\right) .
$$

Observe that in the expression of condition $(T C C P)$ for $\left(f_{1}(\omega, \cdot, \cdot, \cdot, \cdot), f_{2}(\omega, \cdot, \cdot, \cdot, \cdot)\right)$, the functions $\bar{f}_{i}, \bar{y}_{i}, \bar{\rho}_{i}$, and $\underline{f}_{i}, \underline{y}_{i}, \underline{\rho}_{i}$ may depend on $\omega$ (we sometimes omit it to shorten the notation), and that $\left(F_{1, \varepsilon}(\omega, \cdot, \cdot, \cdot), F_{2, \varepsilon}(\omega, \cdot, \cdot, \cdot)\right)$ is a TCSVR-functional whose condition $(T C C P)$ is exactly that of $\left(F_{1}(\omega, \cdot, \cdot, \cdot), F_{2}(\omega, \cdot, \cdot, \cdot)\right)$, i.e. with $\bar{f}_{i}, \bar{y}_{i}, \bar{\rho}_{i}$, and $\underline{f}_{i}, \underline{y}_{i}, \underline{\rho}_{i}$. Since $\underline{y}_{i}$ and $\bar{y}_{i}$ do not depend on $\varepsilon$, condition (3.4) is automatically satisfied. Condition (3.3) holds for $\mathbf{P}$-a.s. $\omega \in \Sigma$. More precisely, in Lemma 
1. Lemma 5.1] we obtained the following estimates for P-a.s. $\omega \in \Sigma$, and for $i=1,2$ :

$$
\begin{aligned}
& \limsup _{\varepsilon \rightarrow 0} \int_{0}^{T}\left\|\frac{d r_{i}}{d t}\left(\omega, t, \frac{\cdot}{\varepsilon}\right)\right\|_{L^{2}\left(\Omega, \mathbb{R}^{l}\right)} d t \leq\left[T \mathcal{L}_{N}(\Omega) \mathbf{E}^{\mathcal{I}} \int_{0}^{T}\left\|\frac{d r_{i}}{d \tau}(\omega, \tau, \cdot)\right\|_{L^{2}\left(Y, \mathbb{R}^{l}\right)}^{2} d \tau\right]^{\frac{1}{2}}, \\
& \limsup _{\varepsilon \rightarrow 0} \int_{0}^{T}\left\|\frac{d q_{i}}{d t}\left(\omega, t, \frac{\cdot}{\varepsilon}\right)\right\|_{L^{2}(\Omega)} d t \leq\left[T \mathcal{L}_{N}(\Omega) \mathbf{E}^{\mathcal{I}} \int_{0}^{T}\left\|\frac{d q_{i}}{d t}(\omega, \tau, \cdot)\right\|_{L^{2}(Y)}^{2} d \tau\right]^{\frac{1}{2}} .
\end{aligned}
$$

Finally we assume that 3.5 holds for $\mathbf{P}$-a.e. $\omega \in \Sigma$, i.e.

$$
a_{i} \underline{\rho}_{i}(\omega) \leq \phi_{i} \leq a_{i} \bar{\rho}_{i}(\omega) .
$$

5.3. Almost sure convergence to the homogenized system. Under above conditions, by combining Theorem 3.1 together with the variational convergence of the sequence of random energies $\Phi_{i, \varepsilon}$ specified above, we intend to analyze the asymptotic behavior in $C\left(0, T, L^{2}(\Omega)\right) \times C\left(0, T, L^{2}(\Omega)\right)$ of the solution $\left(u_{\varepsilon}(\omega), v_{\varepsilon}(\omega)\right)$ of the random reaction-diffusion system when $\varepsilon \rightarrow 0$ :

$$
\left(\mathcal{S}_{\varepsilon}(\omega)\right)\left\{\begin{array}{l}
\frac{d u_{\varepsilon}(\omega)}{d t}(t)+A_{1, e}(\omega)\left(u_{\varepsilon}(\omega, t)\right)=F_{1, \varepsilon}\left(\omega, t, u_{\varepsilon}(\omega, t), v_{\varepsilon}(\omega, t)\right) \text { for a.e. } t \in(0, T), \\
\frac{d v_{\varepsilon}(\omega)}{d t}(t)+A_{2, e}(\omega)\left(u_{\varepsilon}(\omega, t)\right)=F_{2, \varepsilon}\left(\omega, t, u_{\varepsilon}(\omega, t), v_{\varepsilon}(\omega, t)\right) \text { for a.e. } t \in(0, T), \\
u_{\varepsilon}(\omega, 0)=u_{\varepsilon}^{0}(\omega), v_{\varepsilon}(\omega, 0)=v_{\varepsilon}^{0}(\omega), \underline{\rho}_{1}(\omega) \leq u_{\varepsilon}(\omega) \leq \bar{\rho}_{1}(\omega), \underline{\rho}_{2}(\omega) \leq v_{\varepsilon}(\omega) \leq \bar{\rho}_{2}(\omega) \\
a_{1} u_{\varepsilon}(\omega, t)+\operatorname{div} D_{\xi} W_{1}\left(\omega, \dot{\bar{\varepsilon}}, \nabla u_{\varepsilon}(\omega, t)\right) \cdot \mathbf{n}=\phi_{1} \text { on } \partial \Omega \text { for all } t \in[0, T] \\
a_{2} v_{\varepsilon}(\omega, t)+\operatorname{div} D_{\xi} W_{2}\left(\omega, \dot{\bar{\varepsilon}}, \nabla v_{\varepsilon}(\omega, t)\right) \cdot \mathbf{n}=\phi_{2} \text { on } \partial \Omega \text { for all } t \in[0, T]
\end{array}\right.
$$

where we have expressed the domain of the subdifferential of each functional $\Phi_{i}(\omega)$.

Theorem 5.1. For each $\omega \in \Sigma$, let denote by $\left(u_{\varepsilon}(\omega), v_{\varepsilon}(\omega)\right)$ the unique solution in $C\left([0, T], L^{2}(\Omega)\right) \times$ $C\left([0, T], L^{2}(\Omega)\right)$ of the reaction-diffusion system $\left(\mathcal{S}_{\varepsilon}(\omega)\right)$. Assume that for $\mathbf{P}$-a.s. $\omega \in \Sigma,\left(u_{\varepsilon}^{0}(\omega), v_{\varepsilon}^{0}(\omega)\right)$ strongly converges to $\left(u^{0}(\omega), v^{0}(\omega)\right)$ in $L^{2}(\Omega)$ and that $\sup _{\varepsilon} \Phi_{i, \varepsilon}\left(\eta_{\varepsilon}(\omega, 0)\right)<+\infty$. Then, for $\mathbf{P}$-a.s. $\omega \in \Sigma,\left(u_{\varepsilon}(\omega), v_{\varepsilon}(\omega)\right)$ uniformly converges in $C\left([0, T], L^{2}(\Omega)\right) \times C\left([0, T], L^{2}(\Omega)\right)$ to the unique solution of the reaction-diffusion system

$$
\left(\mathcal{S}^{h o m}(\omega)\right)\left\{\begin{array}{l}
\frac{d u(\omega)}{d t}(t)+A_{1}^{\text {hom }}(\omega)(u(\omega, t)) \ni F_{1}^{\text {hom }}(\omega, t, u(\omega, t), v(\omega, t)) \text { for a.e. } t \in(0, T) \\
\frac{d v(\omega)}{d t}(t)+A_{2}^{\text {hom }}(\omega)(v(\omega, t)) \ni F_{2}^{h o m}(\omega, t, u(\omega, t), v(\omega, t)) \text { for a.e. } t \in(0, T) \\
u(\omega, 0)=u^{0}(\omega), v(\omega, 0)=v^{0}(\omega), \underline{\rho}_{1}(\omega) \leq u(\omega) \leq \bar{\rho}_{1}(\omega), \underline{\rho}_{2}(\omega) \leq v(\omega) \leq \bar{\rho}_{2}(\omega), \\
a_{1} u(\omega, t)+\operatorname{div} D_{\xi} W_{1}^{h o m}(\omega, \nabla u(\omega, t)) \cdot \mathbf{n} \ni \phi_{1} \text { on } \partial \Omega \text { for all } t \in[0, T], \\
a_{2} v(\omega, t)+\operatorname{div} D_{\xi} W_{2}^{h o m}(\omega, \nabla v(\omega, t)) \cdot \mathbf{n} \ni \phi_{2} \text { on } \partial \Omega \text { for all } t \in[0, T],
\end{array}\right.
$$

where $F_{i}^{\text {hom }}$ is given by $F_{i}^{\text {hom }}(\omega, t, u, v)(x)=f_{i}^{\text {hom }}(\omega, t, x, u(x), v(x))$ with

$$
\begin{aligned}
& f_{1}^{\text {hom }}\left(\omega, t, \zeta, \zeta^{\prime}\right)=r_{1}^{\text {hom }}(\omega, t) \odot h_{1}\left(\zeta^{\prime}\right) \cdot g_{1}(\zeta)+q_{1}^{\text {hom }}(\omega, t), \\
& f_{2}^{\text {hom }}\left(\omega, t, \zeta, \zeta^{\prime}\right)=r_{2}^{\text {hom }}(\omega, t) \odot h_{2}(\zeta) \cdot g_{2}\left(\zeta^{\prime}\right)+q_{2}^{\text {hom }}(\omega, t) \\
& r_{i}^{\text {hom }}(\omega, t)=\mathbf{E}^{\mathcal{F}}\left(\int_{(0,1)^{N}} r_{i}(\omega, t, y) d y\right), \\
& q_{i}^{\text {hom }}(\omega, t)=\mathbf{E}^{\mathcal{F}}\left(\int_{(0,1)^{N}} q_{i}(\omega, t, y) d y\right) .
\end{aligned}
$$


Moreover, for $\mathbf{P}$-a.e. $\omega \in \Sigma,\left(\frac{d u_{\varepsilon}(\omega)}{d t}, \frac{d v_{\varepsilon}(\omega)}{d t}\right) \rightarrow\left(\frac{d u(\omega)}{d t}, \frac{d u(\omega)}{d t}\right)$ weakly in $L^{2}\left(0, T, L^{2}(\Omega)\right) \times L^{2}\left(0, T, L^{2}(\Omega)\right)$ and $\underline{y}_{1}(\omega, T) \leq u(\omega) \leq \bar{y}_{1}(\omega, T), \underline{y}_{2}(\omega, T) \leq v(\omega) \leq \bar{y}_{2}(\omega, T)$.

When the the dynamical system $\left(\Sigma, \mathcal{A}, \mathbf{P},\left(T_{z}\right)_{z \in \mathbb{Z}^{N}}\right)$ is ergodic, the initial conditions are deterministic, i.e. $u_{\varepsilon}^{0}(\omega)=u_{\varepsilon}^{0}$ and $v_{\varepsilon}^{0}(\omega)=v_{\varepsilon}^{0}$ for $\mathbf{P}$-a.s. $\omega \in \Sigma$, together with $\underline{\rho}_{i}, \bar{\rho}_{i}, \underline{f}_{i}$, and $\bar{f}_{i}$, then $\left(\mathcal{S}^{\text {hom }}\right)$ is deterministic and the expectation operator must be replaced by the mathematical expectation operator in formulas expressing $\bar{r}_{i}$ and $\bar{q}_{i}$.

If in addition $W_{i}$ satisfies $\left(\mathrm{D}_{3}^{*}\right)$, then $\operatorname{div} \partial_{\xi} W_{i}^{\text {hom }}(\omega, \nabla u(t))$ or $\operatorname{div} \partial_{\xi} W_{i}^{\text {hom }}(\nabla u(t))$ are univalent equal to $\operatorname{div} D_{\xi} W_{i}^{\text {hom }}(\omega, \nabla u(t))$ or $\operatorname{div} D_{\xi} W_{i}^{\text {hom }}(\nabla u(t))$, and the differential inclusions are equalities.

Proof. The proof is a straightforward consequence of Theorem 3.1, and [1, Lemma 5.1, Lemma 5.2].

5.4. The case of a coupling between a random r.d.e. and a random n.d.r.e. We place ourselves within the framework of Section 4 . We assume that the random reaction functional $F_{1}$ fulfills the conditions of Section 5.2 and that $f_{2}$ does not depend on the space variable. Note that under these conditions, $F_{2, \varepsilon}(\omega, \cdot, \cdot, \cdot)=F_{2}(\omega, \cdot, \cdot, \cdot)$. Theorem 5.2 below whose proof is a direct consequence of Theorem 4.1 and [1, Lemma 5.1, Lemma 5.2], expresses the homogenized problem of the following random system:

$$
\left(\mathcal{S}_{\varepsilon}(\omega)\right)\left\{\begin{array}{l}
\frac{d u_{\varepsilon}(\omega)}{d t}(t)+A_{e}(\omega)\left(u_{\varepsilon}(\omega, t)\right)=F_{1, \varepsilon}\left(\omega, t, u_{\varepsilon}(\omega, t), v_{\varepsilon}(\omega, t)\right) \text { for a.e. } t \in(0, T) \\
\frac{d v_{\varepsilon}(\omega)}{d t}(t)=F_{2}\left(\omega, t, u_{\varepsilon}(\omega, t), v_{\varepsilon}(\omega, t)\right) \text { for a.e. } t \in(0, T) \\
u_{\varepsilon}(\omega, 0)=u_{\varepsilon}^{0}(\omega), v_{\varepsilon}(\omega, 0)=v_{\varepsilon}^{0}(\omega), \underline{\rho}_{1}(\omega) \leq u_{\varepsilon}(\omega) \leq \bar{\rho}_{1}(\omega), \underline{\rho}_{2}(\omega) \leq v_{\varepsilon}(\omega) \leq \bar{\rho}_{2}(\omega) \\
a_{1} u_{\varepsilon}(\omega, t)+\operatorname{div} D_{\xi} W_{1}\left(\omega, \dot{\bar{\varepsilon}}, \nabla u_{\varepsilon}(\omega, t)\right) \cdot \eta=\phi_{1} \text { on } \partial \Omega \text { for all } t \in[0, T]
\end{array}\right.
$$

Theorem 5.2. For each $\omega \in \Sigma$, let denote by $\left(u_{\varepsilon}(\omega), v_{\varepsilon}(\omega)\right)$ the unique solution in $C\left([0, T], L^{2}(\Omega)\right) \times$ $C\left([0, T], L^{2}(\Omega)\right)$ of the system $\left(\mathcal{S}_{\varepsilon}(\omega)\right)$. Assume that for $\mathbf{P}$-a.s. $\omega \in \Sigma,\left(u_{\varepsilon}^{0}(\omega), v_{\varepsilon}^{0}(\omega)\right)$ strongly converges to $\left(u^{0}(\omega), v^{0}(\omega)\right)$ in $L^{2}(\Omega)$ and that $\sup _{\varepsilon} \Phi_{\varepsilon}\left(\eta_{\varepsilon}(\omega, 0)\right)<+\infty$. Then, for $\mathbf{P}$-a.s. $\omega \in \Sigma$, $\left(u_{\varepsilon}(\omega), v_{\varepsilon}(\omega)\right)$ uniformly converges in $C\left([0, T], L^{2}(\Omega)\right) \times C\left([0, T], L^{2}(\Omega)\right)$ to the unique solution of the system

$$
\left(\mathcal{S}^{h o m}(\omega)\right)\left\{\begin{array}{l}
\frac{d u(\omega)}{d t}(t)+A^{\text {hom }}(\omega)(u(\omega, t)) \ni F_{1}^{\text {hom }}(\omega, t, u(\omega, t), v(\omega, t)) \text { for a.e. } t \in(0, T) \\
\frac{d v(\omega)}{d t}(t)=F_{2}(\omega, t, u(\omega, t), v(\omega, t)) \text { for a.e. } t \in(0, T) \\
u(\omega, 0)=u^{0}(\omega), v(\omega, 0)=v^{0}(\omega), \underline{\rho}_{1}(\omega) \leq u(\omega) \leq \bar{\rho}_{1}(\omega), \underline{\rho}_{2}(\omega) \leq v(\omega) \leq \bar{\rho}_{2}(\omega), \\
a_{1} u(\omega, t)+\operatorname{div} D_{\xi} W_{1}^{h o m}(\omega, \nabla u(\omega, t)) \cdot \mathbf{n} \ni \phi_{1} \text { on } \partial \Omega \text { for all } t \in[0, T],
\end{array}\right.
$$

where $F_{1}^{\text {hom }}$ is given by $F_{1}^{\text {hom }}(\omega, t, u, v)(x)=f_{i}^{h o m}(\omega, t, x, u(x), v(x))$ with

$$
\begin{aligned}
& f_{1}^{\text {hom }}\left(\omega, t, \zeta, \zeta^{\prime}\right)=r_{1}^{\text {hom }}(\omega, t) \odot h_{1}\left(\zeta^{\prime}\right) \cdot g_{1}(\zeta)+q_{1}^{\text {hom }}(\omega, t), \\
& r_{1}^{\text {hom }}(\omega, t)=\mathbf{E}^{\mathcal{F}}\left(\int_{(0,1)^{N}} r_{1}(\omega, t, y) d y\right), \\
& q_{1}^{\text {hom }}(\omega, t)=\mathbf{E}^{\mathcal{F}}\left(\int_{(0,1)^{N}} q_{1}(\omega, t, y) d y\right) .
\end{aligned}
$$

Moreover, for $\mathbf{P}$-a.e. $\omega \in \Sigma,\left(\frac{d u_{\varepsilon}(\omega)}{d t}, \frac{d v_{\varepsilon}(\omega)}{d t}\right) \rightarrow\left(\frac{d u(\omega)}{d t}, \frac{d u(\omega)}{d t}\right)$ weakly in $L^{2}\left(0, T, L^{2}(\Omega)\right) \times L^{2}\left(0, T, L^{2}(\Omega)\right)$ and $\underline{y}_{1}(\omega, T) \leq u(\omega) \leq \bar{y}_{1}(\omega, T), \underline{y}_{2}(\omega, T) \leq v(\omega) \leq \bar{y}_{2}(\omega, T)$. 
When the the dynamical system $\left(\Sigma, \mathcal{A}, \mathbf{P},\left(T_{z}\right)_{z \in \mathbb{Z}^{N}}\right)$ is ergodic, the initial conditions are deterministic, i.e. $\left(u_{\varepsilon}^{0}(\omega), v_{\varepsilon}^{0}(\omega)\right)=\left(u_{\varepsilon}^{0}, v_{\varepsilon}^{0}\right)$ for $\mathbf{P}$-a.e. $\omega \in \Sigma, F_{2}$ is deterministic together with $\underline{\rho}_{i}, \bar{\rho}_{i}, \underline{f}_{i}$, and $\bar{f}_{i}$, then $\left(\mathcal{S}^{\text {hom }}\right)$ is deterministic and the expectation operator must be replaced by the mathematical expectation operator in formulas expressing $\bar{r}_{1}^{\text {hom }}$ and $\bar{q}_{1}^{\text {hom }}$.

If in addition $W$ satisfies $\left(\mathrm{D}_{3}\right)$, then $\partial_{\xi} W^{\text {hom }}(\omega, \nabla u(t))$ or $\partial_{\xi} W^{\text {hom }}(\nabla u(t))$ are univalent equal to $D_{\xi} W^{\text {hom }}(\omega, \nabla u(t))$ or $D_{\xi} W^{h o m}(\nabla u(t))$, and the differential inclusions are equalities.

Remark 5.1. By applying Theorem 4.2 and [1, Lemma 5.1, Lemma 5.2], one can easily express the homogenized problem of a random system of two non diffusive reaction equations with obvious adaptations.

5.5. Application to stochastic homogenization of a prey-predator random model with saturation effect. For the notation refer to Example 2.3. For each $i=1,2$, we are given two functions $W_{i}^{ \pm}$in $\operatorname{Conv}_{\alpha, \beta, \gamma}$, where $W_{i}^{-}, W_{i}^{+}$do not depend on $x$, and satisfy $\left(\mathrm{D}_{3}^{*}\right)$, and two functions $\alpha_{i}^{ \pm}:[0, T] \rightarrow(0,+\infty)$ in $W^{1,2}(0, T)$ for which there exist positive real numbers $\underline{\alpha}_{1}^{ \pm}, \underline{\alpha}_{2}^{ \pm}$, and $\bar{\alpha}_{2}^{ \pm}$such that

$$
\left\{\begin{array}{l}
\alpha_{1}^{ \pm}(t) \geq \underline{\alpha}_{1}^{ \pm}>0 \\
\bar{\alpha}_{2}^{ \pm} \geq \alpha_{2}^{ \pm}(t) \geq \underline{\alpha}_{2}^{ \pm}>0
\end{array}\right.
$$

We also consider two functions $K^{ \pm}:[0, T] \rightarrow(0,+\infty)$ in $W^{1,2}(0, T)$ satisfying $K(t)^{ \pm} \geq \underline{K}^{ \pm}>0$ for some positive real numbers $\underline{K}^{ \pm}$, and two functions $a^{ \pm}:[0, T] \rightarrow(0,+\infty)$ in $W^{1,2}(0, T)$ for which there exist a constant $\bar{a}^{ \pm}>0$ such that $\bar{a}^{ \pm} \geq a^{ \pm}(t)>0$.

We now consider the random environment described in [1, Appendix $B_{2}$ ] with $N=2$. Recall that the spherical heterogeneities of size of order $\varepsilon$ have centers independently randomly distributed with a given frequency $\lambda$, following a Poisson point process with intensity $\lambda$. This random environment is modeled by an ergodic dynamical system $\left(\Sigma, \mathcal{A}, \mathbf{P}_{\lambda},\left(T_{x}\right)_{x \in \mathbb{R}^{2}}\right)$ where $T_{x} \omega=\omega-x$, and, for every bounded Borel set $B$, and every $k \in \mathbb{N}$,

$$
\mathbf{P}_{\lambda}(\#(\Sigma \cap B)=k)=\lambda^{k} \mathcal{L}_{2}(B)^{k} \frac{\exp \left(-\lambda \mathcal{L}_{N}(B)\right)}{k !}
$$

so that $\mathbf{E}_{\lambda}[\# \Sigma \cap B]=\lambda \mathcal{L}_{N}(B)$. Given $R>0$, define the random density $W_{i}$ associated with the random diffusion part, by

$$
W_{i}(\omega, x, \xi)= \begin{cases}W_{i}^{-}(\xi) & \text { if } x \in \bigcup_{i \in \mathbb{N}} B_{R}\left(\omega_{i}\right) \\ W_{i}^{+}(\xi) & \text { otherwise. }\end{cases}
$$

Similarly we set

$$
\begin{aligned}
& \alpha_{i}(\omega, t, x)=\left\{\begin{array}{l}
\alpha_{i}^{-}(t) \text { if } x \in \bigcup_{j \in \mathbb{N}} B_{R}\left(\omega_{j}\right), \\
\alpha_{i}^{+}(t) \text { otherwise. }
\end{array}\right. \\
& K_{\text {car }}(\omega, t, x)=\left\{\begin{array}{l}
K^{-}(t) \text { if } x \in \bigcup_{j \in \mathbb{N}} B_{R}\left(\omega_{j}\right), \\
K^{+}(t) \text { otherwise. }
\end{array}\right. \\
& a(\omega, t, x)=\left\{\begin{array}{l}
a^{-}(t) \text { if } x \in \bigcup_{j \in \mathbb{N}} B_{R}\left(\omega_{j}\right), \\
a^{+}(t) \text { otherwise. }
\end{array}\right.
\end{aligned}
$$

We define the following constants

$$
\begin{aligned}
& \underline{\alpha}_{i}=\inf _{t \in[0,+\infty)} \min \left(\alpha_{i}^{-}(t), \alpha_{i}^{+}(t)\right), i=1,2 ; \\
& \bar{\alpha}_{2}=\sup _{t \in[0,+\infty)} \max \left(\alpha_{2}^{-}(t), \alpha_{2}^{+}(t)\right) ; \\
& \bar{a}=\sup _{t \in[0,+\infty)} \max \left(a^{-}(t), a^{+}(t)\right) ; \\
& \underline{K}=\inf _{t \in[0,+\infty)} \min \left(K^{-}(t), K^{+}(t)\right) .
\end{aligned}
$$


Let $b$ and $c$ be two positive constants and assume that the the extinction threshold satisfies $\mu_{\text {ext }}:=$ $c \frac{\underline{\alpha}_{1} \underline{\alpha}_{2}}{\overline{a \alpha_{2}}} \geq 4$. Then, choosing $\underline{\rho}_{1}, \bar{\rho}_{1}$ and $\bar{\rho}_{2}$ fulfilling (2.3), we consider the following system stemming from Example 2.3:

$$
\begin{aligned}
& \int \frac{d u_{\varepsilon}}{d t}(\omega, t)-\operatorname{div} D_{\xi} W_{1}\left(\omega, \frac{\dot{-}}{\varepsilon}, \nabla u_{\varepsilon}(\omega, t)\right)=\alpha_{1}(\omega, t, \dot{\dot{\varepsilon}}) u_{\varepsilon}(\omega, t)\left(1-\frac{u_{\varepsilon}(\omega, t)}{K_{\text {car }}(\omega, t, \dot{\bar{\varepsilon}})}\right) \\
& -a(\omega, t, \dot{\bar{\varepsilon}}) v_{\varepsilon}(\omega, t)\left(1-\exp \left(-b u_{\varepsilon}(\omega, t)\right)\right) \\
& \text { for a.e. } t \in(0, T) \text {, } \\
& \left(\mathcal{S}_{\varepsilon}(\omega)\right)\left\{\begin{array}{r}
\frac{d v_{\varepsilon}(\omega)}{d t}(t)-\operatorname{div} D_{\xi} W_{2}\left(\omega, \frac{\cdot}{\varepsilon}, \nabla v_{\varepsilon}(\omega, t)\right)=\alpha_{2}\left(\omega, t, \frac{\cdot}{\varepsilon}\right) v_{\varepsilon}(\omega, t)\left(1-c \frac{v_{\varepsilon}(\omega, t)}{u_{\varepsilon}(\omega, t)}\right) \\
\text { for a.e. } t \in(0, T),
\end{array}\right. \\
& u_{\varepsilon}(\omega, 0)=u_{\varepsilon}^{0}(\omega) \in H^{1}(\Omega), v_{\varepsilon}(\omega, 0)=v_{\varepsilon}^{0}(\omega) \in H^{1}(\Omega), \underline{\rho}_{1} \leq u_{\varepsilon}^{0}(\omega) \leq \bar{\rho}_{1}, 0 \leq v_{\varepsilon}^{0}(\omega) \leq \bar{\rho}_{2}, \\
& u_{\varepsilon}(\omega, t)=0 \text { on } \Gamma_{1}, D_{\xi} W_{1}\left(\omega, \dot{\bar{\varepsilon}}, \nabla u_{\varepsilon}(\omega, t)\right) \cdot \mathbf{n}=0 \text { on } \partial \Omega \backslash \Gamma_{1}, \\
& v_{\varepsilon}(\omega, t)=0 \text { on } \Gamma_{2}, D_{\xi} W_{2}\left(\omega, \dot{\bar{\varepsilon}}, \nabla v_{\varepsilon}(\omega, t)\right) \cdot \mathbf{n}=0 \text { on } \partial \Omega \backslash \Gamma_{2} \text {. }
\end{aligned}
$$

According to Proposition 2.3 and Theorem 2.1. $\left(\mathcal{S}_{\varepsilon}(\omega)\right)$ admits a unique solution $\left(u_{\varepsilon}(\omega, \cdot), v_{\varepsilon}(\omega, \cdot)\right)$ in the space $C\left([0, T], L^{2}(\Omega)\right) \times C\left([0, T], L^{2}(\Omega)\right)$, which satisifies $\rho_{1} \leq u_{\varepsilon}(\omega, t) \leq \bar{\rho}_{1} \exp \left(\bar{\alpha}_{1} t\right)$ and $0 \leq v_{\varepsilon}(\omega, t) \leq \bar{\rho}_{2}$ for all $t \in[0, T]$. Furthermore, $u_{\varepsilon}(\omega, \cdot)$ and $v_{\varepsilon}(\omega, \cdot)$ admit a right derivative at each $t \in(0, T)$. The system models the evolution of two species with density $u_{\varepsilon}(\omega, \cdot)$ and $v_{\varepsilon}(\omega, \cdot)$ of a prey and a predator respectively, whose birth growth rate, maximum carrying capacity, and saturation effect, take two values at random depending on whether the species reside in the environment made up of the union of small balls of size $\varepsilon$ or not (refer to the comments of Example 2.3). The homogenized system is expressed in the Proposition below. It is interesting to note that the effective growth rate $\alpha_{i}^{\text {hom }}$ of each two species is the mean value of $\alpha_{i}$ with respect to the product probability measure $\mathcal{L}_{2}\left\lfloor(0,1)^{2} \otimes \mathbf{P}_{\lambda}\right.$, while the effective maximum carrying capacity $K_{\text {car }}^{\text {hom }}$ is now a function of the growth rate $\alpha_{1}$ and $K_{\text {car }}$, illustrating the interplay between the growth rate of the prey and the maximum carrying capacity of the environment when the size of the spatial heterogeneities, with a constant frequency $\lambda$, is very small compared with the size of the domain 5 .

Proposition 5.1. Assume that the initial conditions are deterministic, that $\left(u_{\varepsilon}^{0}, v_{\varepsilon}^{0}\right)$ strongly converges to $\left(u^{0}, v^{0}\right)$ in $L^{2}(\Omega)$ and that $\sup _{\varepsilon} \Phi_{1, \varepsilon}\left(u_{\varepsilon}^{0}\right)<+\infty, \sup _{\varepsilon} \Phi_{1, \varepsilon}\left(u_{\varepsilon}^{0}\right)<+\infty$. Then, for $\mathbf{P}$-a.s. $\omega \in \Sigma$, $\left(u_{\varepsilon}, v_{\varepsilon}\right)$ uniformly converges in $C\left([0, T], L^{2}(\Omega)\right) \times C\left([0, T], L^{2}(\Omega)\right)$ to the unique solution $(u, v)$ of the deterministic reaction-diffusion system

$$
(\mathcal{S})\left\{\begin{array}{l}
\frac{d u}{d t}(t)-\operatorname{div} D_{\xi} W_{1}^{h o m}(\nabla u(t))=\alpha_{1}^{h o m}(t) u(t)\left(1-\frac{u(t)}{K_{c a r}^{h o m}(t)}\right) \\
\frac{d v}{d t}(t)-\operatorname{div} D_{\xi} W_{2}^{h o m}(\nabla v(t))=\alpha_{2}^{h o m}(t) v(t)\left(1-c \frac{v(t)}{u(t)}\right) \quad \text { for a.e. } t \in(0, T), \\
u(0)=u^{0} \in H^{1}(\Omega), v(0)=v^{0} \in H^{1}(\Omega), \underline{\rho}_{1} \leq u^{0} \leq \bar{\rho}_{1}, 0 \leq v^{0} \leq \bar{\rho}_{2}, \\
u(t)=0 \text { on } \Gamma_{1}, D_{\xi} W_{1}^{h o m}(\nabla u(t)) \cdot \mathbf{n}=0 \text { on } \partial \Omega \backslash \Gamma_{1}, \\
v(t)=0 \text { on } \Gamma_{2}, D_{\xi} W_{2}^{h o m}(\nabla v(t)) \cdot \mathbf{n}=0 \text { on } \partial \Omega \backslash \Gamma_{2}
\end{array}\right.
$$

\footnotetext{
${ }^{5}$ To shorten the notation, for $i=1,2$, we assume that $W_{i}^{ \pm}$satisfy $\left(\mathrm{D}_{3}^{*}\right)$ so that $W_{i}^{\text {hom }}$ is Gâteaux differentiable.
} 
where

$$
\begin{aligned}
\alpha_{i}^{\text {hom }}(t) & =\mathbf{E}\left(\int_{(0,1)^{2}} \alpha_{i}(\cdot, t, y) d y\right) \\
& =\alpha_{i}^{-}(t)+\left(\alpha_{i}^{+}(t)-\alpha_{i}^{-}(t)\right) \exp \left(-\lambda \pi R^{2}\right) i=1,2 \\
a^{\text {hom }}(t) & =\mathbf{E}\left(\int_{(0,1)^{2}} a(\cdot, t, y) d y\right) \\
& =a^{-}(t)+\left(a^{+}(t)-a^{-}(t)\right) \exp \left(-\lambda \pi R^{2}\right) \\
K_{c a r}^{h o m}(t) & =\frac{\mathbf{E}\left(\int_{(0,1)^{2}} \alpha_{1}(\cdot, t, y) d y\right)}{\mathbf{E}\left(\int_{(0,1)^{2}} \frac{\alpha_{1}(\cdot, t, y)}{K_{c a r}(\cdot, t, y)} d y\right)} \\
& =\frac{\alpha_{1}^{-}(t)+\left(\alpha_{1}^{+}(t)-\alpha_{1}^{-}(t)\right) \exp \left(-\lambda \pi R^{2}\right)}{\frac{\alpha_{1}^{-}(t)}{K^{-}(t)}+\left(\frac{\alpha_{1}^{+}(t)}{K^{+}(t)}-\frac{\alpha_{1}^{-}(t)}{K^{-}(t)}\right) \exp \left(-\lambda \pi R^{2}\right)}
\end{aligned}
$$

Furthermore $\underline{\rho}_{1} \leq u \leq \bar{\rho}_{1} \exp \left(\bar{\alpha}_{1} T\right)$ and $0 \leq v \leq \bar{\rho}_{2}$.

Proof. Apply Theorem 5.1 with

$$
\begin{aligned}
f_{1}\left(\omega, t, x, \zeta, \zeta^{\prime}\right) & =\alpha_{1}(\omega, t, x) \zeta\left(1-\frac{\zeta}{K_{c a r}(\omega, t, x)}\right)-a(\omega, t, x) \zeta^{\prime}(1-\exp (-b \zeta)) \\
& =\alpha_{1}(\omega, t, x) \zeta-\frac{\alpha_{1}(\omega, t, x)}{K_{c a r}(\omega, t, x)} \zeta^{2}-a(\omega, x, t) \zeta^{\prime}(1-\exp (-b \zeta)), \\
f_{2}\left(\omega, t, x, \zeta, \zeta^{\prime}\right) & =\alpha_{2}(\omega, t, x) \zeta^{\prime}-c \alpha_{2}(\omega, t, x) \frac{\zeta^{\prime 2}}{\zeta}
\end{aligned}
$$

Then

$$
\begin{array}{r}
f_{1}^{h o m}\left(t, \zeta, \zeta^{\prime}\right)=\mathbf{E}\left(\int_{(0,1)^{2}} \alpha_{1}(\cdot, t, y) d y\right) \zeta-\mathbf{E}\left(\int_{(0,1)^{2}} \frac{\alpha_{1}(\cdot, t, y)}{K_{c a r}(\cdot, t, y)} d y\right) \zeta^{2} \\
-\mathbf{E}\left(\int_{(0,1)^{2}} a(\cdot, t, y) d y\right) \zeta^{\prime}(1-\exp (-b \zeta))
\end{array}
$$

which can be written, according to the structure of the reaction function of the system $\left(\mathcal{S}_{\varepsilon}\right)$,

$$
\begin{array}{r}
f_{1}^{h o m}\left(t, \zeta, \zeta^{\prime}\right)= \\
\mathbf{E}\left(\int_{(0,1)^{2}} \alpha_{1}(\cdot, t, y) d y\right) \zeta\left(1-\frac{\mathbf{E}\left(\int_{(0,1)^{2}} \frac{\alpha_{1}(\cdot, t, y)}{K_{c a r}(\cdot, t, y)} d y\right)}{\mathbf{E}\left(\int_{(0,1)^{2}} \alpha_{1}(\cdot, t, y) d y\right)} \zeta\right) \\
=\alpha_{1}^{h o m}(t) \zeta\left(1-\frac{\zeta}{K_{c a r}^{\text {hom }}(t)}\right)-a^{h o m}(t) \zeta^{\prime}(1-\exp (-b \zeta)) .
\end{array}
$$

Similarly we have

$$
\begin{aligned}
f_{2}^{h o m}\left(t, \zeta, \zeta^{\prime}\right) & =\alpha_{2}^{h o m}(t) \zeta^{\prime}-c \alpha_{2}^{h o m}(t) \frac{\zeta^{\prime 2}}{\zeta} \\
& =\alpha_{2}^{h o m}(t) \zeta^{\prime}\left(1-c \frac{\zeta^{\prime}}{\zeta}\right) .
\end{aligned}
$$


It remains to compute $\alpha_{i}^{\text {hom }}, a^{\text {hom }}$ and $K_{c a r}^{\text {hom }}$. Observe the equivalence

$$
\exists \omega \in \Sigma \text { s. t. } y \in \bigcup_{i \in \mathbb{N}} B_{R}\left(\omega_{i}\right) \Longleftrightarrow \#\left(\Sigma \cap B_{R}(y)\right) \geq 1 .
$$

Then using Fubini's theorem, we infer that

$$
\begin{aligned}
& \alpha_{i}^{\text {hom }} \\
& =\alpha_{i}^{+}(t) \int_{\Sigma} \int_{(0,1)^{2}} 1_{\left[\#\left(\Sigma \cap B_{R}(y)\right)=0\right]}(\omega, y) d y d \mathbf{P}(\omega)+\alpha_{i}^{-}(t) \int_{\Sigma} \int_{(0,1)^{2}} 1_{\left[\#\left(\Sigma \cap B_{R}(y)\right) \geq 1\right]}(\omega, y) d y d \mathbf{P}(\omega) \\
& =\alpha_{i}^{+}(t) \int_{(0,1)^{2}} \int_{\Sigma} 1_{\left[\#\left(\Sigma \cap B_{R}(y)\right)=0\right]}(\omega, y) d \mathbf{P}(\omega) d y+\alpha_{i}^{-}(t) \int_{(0,1)^{2}} \int_{\Sigma} 1_{\left[\#\left(\Sigma \cap B_{R}(y)\right) \geq 1\right]}(\omega, y) d \mathbf{P}(\omega) d y \\
& =\alpha_{i}^{+}(t) \exp \left(-\lambda \pi R^{2}\right)+\alpha_{i}^{-}(t)\left(\left(1-\exp \left(-\lambda \pi R^{2}\right)\right)\right) \\
& =\alpha_{i}^{-}(t)+\left(\alpha_{i}^{+}(t)-\alpha_{i}^{-}(t)\right) \exp \left(-\lambda \pi R^{2}\right) .
\end{aligned}
$$

We express $a^{\text {hom }}$ and $K_{c a r}^{h o m}$ by a similar calculation.

\section{Appendix A. Proofs of Propositions 2.1 2.5}

A.1. Proof of Proposition 2.1. Clearly the functions $f_{i}$ satisfy the structure condition of TCCPstructured reaction functions with $l=3$ and

$$
\begin{aligned}
& r_{i}(t, x)=\left(\alpha_{i}(t, x),-\frac{\alpha_{i}(t, x)}{K_{i}(t, x)},-a_{i, i+1} \frac{\alpha_{i}(t, x)}{K_{i}(t, x)}\right) \quad\left(\overline{i+1} \in \frac{\mathbf{Z}}{2 \mathbf{Z}}\right), \\
& h_{1}\left(\zeta^{\prime}\right)=\left(1,1, \zeta^{\prime}\right), h_{2}(\zeta)=(1,1, \zeta), \\
& g_{1}(\zeta)=\left(\zeta, \zeta^{2}, \zeta\right), g_{2}\left(\zeta^{\prime}\right)=\left(\zeta^{\prime}, \zeta^{\prime 2}, \zeta^{\prime}\right) .
\end{aligned}
$$

Let us show that condition $(T C C P)$ is fulfilled. Take $\underline{f}_{i}=0$, and $\underline{\rho}_{i}=0$. Then $\underline{y}_{i}=0$ and $f_{1}\left(t, x, \underline{y}_{1}(t), \zeta^{\prime}\right)=0 \geq \underline{f}_{1}\left(t, \underline{y}_{1}(t)\right)$ for all $\zeta^{\prime} \in \mathbb{R}$. Similarly $f_{2}\left(t, x, \zeta, \underline{y}_{2}(t)\right)=0 \geq \underline{f}_{1}\left(t, \underline{y}_{2}(t)\right)$ for all $\zeta \in \mathbb{R}$.

On the other hand take $\bar{f}_{1}(t, \zeta)=\overline{\alpha_{1}} \zeta$, where $\bar{\rho}_{1}>0$ is any real number. Similarly $\bar{f}_{2}\left(t, \zeta^{\prime}\right)=$ $\overline{\alpha_{2}} \zeta^{\prime}$, where $\bar{\rho}_{2}>0$ is any real number. Then $\bar{y}_{i}(t)=\bar{\rho}_{i} \exp \left(\bar{\alpha}_{i} t\right)$ and $f_{1}\left(t, x, \bar{y}_{1}(t), \zeta^{\prime}\right) \leq \bar{\alpha}_{1} \bar{y}_{1}(t)=$ $\bar{f}_{1}\left(t, \bar{y}_{1}(t)\right)$ for all $\zeta^{\prime} \geq 0$. Similarly $f_{2}\left(t, x, \zeta, \bar{y}_{2}(t)\right) \leq \bar{\alpha}_{2} \bar{y}_{2}(t)=\bar{f}_{2}\left(t, \bar{y}_{2}(t)\right)$ for all $\zeta \geq 0$. This proves the claim since $\left[y_{i}(T), \bar{y}_{i}(T)\right]=\left[0, \bar{\rho}_{i} \exp \left(\bar{\alpha}_{i} T\right)\right]$.

A.2. Proof of Proposition 2.2. As in the previous example, the functions $f_{i}$ satisfy the structure condition of TCCP-structured reaction functions with $l=3$ and

$$
\begin{aligned}
& r_{i}(t, x)=\left(\alpha_{i}(t, x),-\frac{\alpha_{i}(t, x)}{K_{i}(t, x)}, b_{i, \overline{i+1}} \frac{\alpha_{i}(t, x)}{K_{i}(t, x)}\right) \quad\left(\overline{i+1} \in \frac{\mathbf{Z}}{2 \mathbf{Z}}\right), \\
& h_{1}\left(\zeta^{\prime}\right)=\left(1,1, \zeta^{\prime}\right), h_{2}(\zeta)=(1,1, \zeta) \\
& g_{1}(\zeta)=\left(\zeta, \zeta^{2}, \zeta\right), g_{2}\left(\zeta^{\prime}\right)=\left(\zeta^{\prime}, \zeta^{\prime 2}, \zeta^{\prime}\right) .
\end{aligned}
$$

Let us show that condition $(T C C P)$ is fulfilled. Take $\underline{f}_{i}=0$, and $\underline{\rho}_{i}=0$. Then $\underline{y}_{i}=0$ and $f_{1}\left(t, x, \underline{y}_{1}(t), \zeta^{\prime}\right)=0 \geq \underline{f}_{1}\left(t, \underline{y}_{1}(t)\right)$ for all $\zeta^{\prime} \in \mathbb{R}$. Similarly $f_{2}\left(t, x, \zeta, \underline{y}_{2}(t)\right)=0 \geq \underline{f}_{1}\left(t, \underline{y}_{2}(t)\right)$ for all $\zeta \in \mathbb{R}$.

Because of the signs positive preceding the b's, we cannot proceed as in the previous example for completing the condition $(T C C P)$. We take $\bar{f}_{i}=0$ and look for $\bar{y}_{i}$ in the form of constants $\bar{\rho}_{i}>0$. For all $\zeta^{\prime} \in\left[0, \bar{\rho}_{2}\right]$ we have

$$
\begin{aligned}
f_{1}\left(t, x, \bar{\rho}_{1}, \zeta^{\prime}\right) & =\alpha_{1}(t, x) \bar{\rho}_{1}\left(1-\frac{\bar{\rho}_{1}}{K_{1}(t, x)}+b_{1,2} \frac{\zeta^{\prime}}{K_{1}(t, x)}\right) \\
& \leq \alpha_{1}(t, x) \bar{\rho}_{1}\left(1-\frac{\bar{\rho}_{1}}{\bar{K}_{1}}+b_{1,2} \frac{\bar{\rho}_{2}}{\underline{K}_{1}}\right) .
\end{aligned}
$$

Take $\bar{\rho}_{1}$ and $\bar{\rho}_{2}$ positive, satisfying

$$
1-\frac{\bar{\rho}_{1}}{\bar{K}_{1}}+b_{1,2} \frac{\bar{\rho}_{2}}{\underline{K}_{1}} \leq 0 .
$$


With this choice, from A.1 and (A.2), we infer that for all $\zeta^{\prime} \in\left[0, \bar{\rho}_{2}\right]$

$$
f_{1}\left(t, x, \bar{y}_{1}(t), \zeta^{\prime}\right) \leq 0=\bar{f}_{1}\left(t, \bar{y}_{1}(t)\right) .
$$

Similarly, with $\bar{\rho}_{1}$ and $\bar{\rho}_{2}$ positive, satisfying

$$
1-\frac{\bar{\rho}_{2}}{\bar{K}_{2}}+b_{2,1} \frac{\bar{\rho}_{1}}{\underline{K}_{2}} \leq 0
$$

for all $\zeta \in\left[0, \bar{\rho}_{1}\right]$, we have

$$
f_{2}\left(t, x, \zeta, \bar{\rho}_{2}\right) \leq 0=\bar{f}_{2}\left(t, \bar{y}_{2}(t)\right) .
$$

Therefore, $\bar{\rho}_{i}>0, i=1,2$, satisfying the system of two inequalities (A.2) and (A.3), i.e.

$$
\begin{gathered}
\bar{\rho}_{1} \geq \frac{\underline{K}_{1} \bar{K}_{1}}{\underline{K}_{1}-b_{1,2} \bar{K}_{1}}, \\
\bar{\rho}_{2} \geq \frac{\underline{K}_{2} \bar{K}_{2}}{\underline{K}_{2}-b_{2,1} \bar{K}_{2}},
\end{gathered}
$$

are suitable for $(T C C P)$ to be fulfilled. It is easy to see that $\bar{\rho}_{1}=\bar{\rho}_{2} \geq \max \left(\frac{\underline{K}_{1} \bar{K}_{1}}{\underline{K}_{1}-b_{1,2} \bar{K}_{1}}, \frac{\underline{K}_{2} \bar{K}_{2}}{\underline{K}_{2}-b_{2,1} \bar{K}_{2}}\right)$ is a solution provided that $\frac{\underline{K}_{1} \bar{K}_{1}}{\underline{K}_{1}-b_{1,2} \bar{K}_{1}}>0$ and $\frac{\underline{K}_{2} \bar{K}_{2}}{\underline{K}_{2}-b_{2,1} \bar{K}_{2}}>0$. These two conditions are ensured by 2.2).

A.3. Proof of Proposition 2.3. Fix $\delta:=\underline{\rho}_{1}>0$ satisfying $0<\underline{\rho}_{1}<\underline{K}$, and chosen later. Set

$$
f_{2, \delta}\left(t, x, \zeta, \zeta^{\prime}\right)=\left\{\begin{array}{l}
f_{2}\left(t, x, \zeta, \zeta^{\prime}\right) \text { if } \zeta \geq \delta \\
f_{2}\left(t, x, \delta, \zeta^{\prime}\right) \text { if } \zeta<\delta .
\end{array}\right.
$$

At the end of the proof, we show that $f_{2, \delta}=f_{2}$ for $\zeta$ and $\zeta^{\prime}$ in suitable intervals. We claim that the pair $\left(f_{1}, f_{2, \delta}\right)$ satisfies the structure condition of TCCP-structured reaction functions with $l=3$ : indeed take

and

$$
\begin{aligned}
& r_{1}(t, x)=\left(\alpha_{1}(t, x), \frac{\alpha_{1}(t, x)}{K_{c a r}(t, x)},-a(t, x)\right), \\
& h_{1}\left(\zeta^{\prime}\right)=\left(1,1, \zeta^{\prime}\right), \\
& g_{1}(\zeta)=\left(\zeta,-\zeta^{2}, 1-\exp (-b \zeta)\right)
\end{aligned}
$$

$$
\begin{aligned}
& r_{2}(t, x)=\left(\alpha_{2}(t, x),-c \alpha_{2}(t, x), 0\right) \text {, } \\
& h_{2, \delta}(\zeta)=\left\{\begin{array}{l}
\left(1, \frac{1}{\zeta}, 0\right) \text { if } \zeta \geq \delta \\
\left(1, \frac{1}{\delta}, 0\right) \text { if } \zeta<\delta,
\end{array}\right. \\
& g_{2}\left(\zeta^{\prime}\right)=\left(\zeta^{\prime}, \zeta^{\prime 2}, 0\right) .
\end{aligned}
$$

It remains to show that $\left(f_{1}, f_{2, \delta}\right)$ fulfills condition $($ TCCP $)$. First take $\underline{\rho}_{2}=0, \underline{f}_{2, \delta}=0$, then $\underline{y}_{2}=\underline{\rho}_{2}$ and $f_{2, \delta}\left(t, x, \zeta, \underline{y}_{2}(t)\right)=0 \geq \underline{f}_{2, \delta}\left(t, \underline{y}_{2}(t)\right)$ for all $\zeta \in \mathbb{R}$. To complete condition $(T C C P)$, we look for

$$
\left\{\begin{array}{l}
\bar{\rho}_{2}>0, \bar{f}_{2, \delta}=0, \bar{y}_{2}=\bar{\rho}_{2} ; \\
\underline{\rho}_{1}, \underline{f}_{1}=0, \underline{y}_{1}=\underline{\rho}_{1} ; \\
\bar{\rho}_{1}>\underline{\rho}_{1}, \bar{f}_{1}(t, \xi)=\bar{\alpha}_{1} \xi, \bar{y}_{1}(t)=\bar{\rho}_{1} \exp \left(\alpha_{1} t\right) .
\end{array}\right.
$$

We first look for $\bar{\rho}_{2}$ satisfying

$$
\begin{aligned}
f_{2, \delta}\left(t, x, \zeta, \bar{\rho}_{2}\right) & \leq \bar{\rho}_{2}\left(\bar{\alpha}_{2}-c \underline{\alpha}_{2} \frac{\bar{\rho}_{2}}{\bar{\rho}_{1}}\right) \\
& \leq 0=\bar{f}_{2}\left(t, \bar{\rho}_{2}\right)
\end{aligned}
$$

for all $\zeta \geq \underline{\rho}_{1}$, which furnishes the first condition:

$$
\frac{\bar{\alpha}_{2}}{\underline{c \alpha}_{2}} \bar{\rho}_{1} \leq \bar{\rho}_{2}
$$


Secondly, we look for $\underline{\rho}_{1}, 0<\underline{\rho}_{1}<\underline{K}$, satisfying

$$
\begin{aligned}
f_{1}\left(t, x, \underline{\rho}_{1}, \zeta^{\prime}\right) & =\alpha_{1}(t, x) \underline{\rho}_{1}\left(1-\frac{\underline{\rho}_{1}}{K_{c a r}(t, x)}\right)-a(x, t) \zeta^{\prime}\left(1-\exp \left(-b \underline{\rho}_{1}\right)\right) \\
& \geq \underline{\alpha}_{1} \underline{\rho}_{1}\left(1-\frac{\underline{\rho}_{1}}{\underline{K}}\right)-\bar{a} \zeta^{\prime} \geq 0
\end{aligned}
$$

for all $\zeta^{\prime} \in\left[0, \bar{\rho}_{2}\right]$, which requires the following second condition

$$
\bar{\rho}_{2} \leq \frac{\underline{\alpha}_{1}}{\bar{a}} \underline{\rho}_{1}\left(1-\frac{\underline{\rho}_{1}}{\underline{K}}\right) .
$$

Combining (A.4) and (A.5), we infer that the choice of $\bar{\rho}_{2}, \bar{\rho}_{1}$ and $\underline{\rho}_{1}$ is conditioned by the following inequality

or equivalently

$$
\frac{\bar{\alpha}_{2}}{c \underline{\alpha}_{2}} \bar{\rho}_{1} \leq \frac{\underline{\alpha}_{1}}{\bar{a}} \underline{\rho}_{1}\left(1-\frac{\underline{\rho}_{1}}{\underline{K}}\right)
$$

$$
\bar{\rho}_{1} \leq \mu_{e x t} \underline{\rho}_{1}\left(1-\frac{\rho_{1}}{\underline{K}}\right)
$$

Hence, we can choose $\bar{\rho}_{2}$ with

$$
\bar{\rho}_{2} \in\left[\frac{\bar{\alpha}_{2}}{c \underline{\alpha}_{2}} \bar{\rho}_{1}, \frac{\underline{\alpha}_{1}}{a} \underline{\rho}_{1}\left(1-\frac{\underline{\rho}_{1}}{\underline{K}}\right)\right] .
$$

which is the last condition in (2.3).

Set $\bar{\rho}_{1}=\theta \underline{\rho}_{1}$ where $\theta \geq \frac{\underline{\underline{\underline{\rho}}}}{\underline{\rho}_{1}}$ (recall that $\left.\frac{\underline{K}}{\underline{\rho}_{1}}>1\right)$. Then $\overline{A .6}$ ) and the previous condition on $\theta$ are equivalent to

$$
\frac{\underline{K}}{\underline{\rho}_{1}} \leq \theta \leq \mu_{e x t}\left(1-\frac{\rho_{1}}{\underline{K}}\right)
$$

so that the possible choice of $\theta$ and $0<\underline{\rho}_{1} \leq \underline{K}$ is governed by

$$
\frac{1}{\mu_{\text {ext }}} \leq \frac{\underline{\rho}_{1}}{\underline{K}}\left(1-\frac{\underline{\rho}}{\underline{K}}\right)
$$

Since $\mu_{e x t} \geq 4$, condition $\left(\right.$ A. 7 is fulfilled by any $\underline{\rho}_{1} \in\left[\underline{K} \frac{1-\sqrt{1-\frac{4}{\mu_{e x t}}}}{2}, \underline{K} \frac{1+\sqrt{1-\frac{4}{\mu_{e x t}}}}{2}\right]$ which is the first condition in (2.3). The choice of $\theta$ is then given by

$$
\theta \in\left[\frac{\underline{K}}{\underline{\rho}}, \mu_{e x t}\left(1-\frac{\underline{\rho}_{1}}{\underline{K}}\right)\right] .
$$

which is the second condition in 2.3 .

It is easily checked that $\bar{\rho}_{1}=\theta \underline{\rho}_{1}$ satisfies

$$
f_{1}\left(t, x, \bar{y}_{1}(t), \zeta^{\prime}\right) \leq \bar{\alpha}_{1} \bar{y}_{1}(t)=\bar{f}_{1}\left(t, \bar{y}_{1}(t)\right)
$$

for all $\xi^{\prime} \in\left[\underline{y}_{2}(T)=0, \bar{\rho}_{2}=\bar{y}_{2}(T)\right]$. Note that $f_{2, \delta}=f_{2}$ for $\left(\zeta, \zeta^{\prime}\right) \in\left[\underline{\rho}_{1}, \bar{\rho}_{1} \exp \left(\bar{\alpha}_{1} t\right)\right] \times\left[0, \bar{\rho}_{2}\right]$. This ends the proof.

A.4. Proof of Proposition 2.4. Clearly the pair $\left(f_{1}, f_{2}\right)$ satisfies the structure condition of TCCPstructured reaction functions with $l=1$. Let us show that $\left(f_{1}, f_{2}\right)$ fulfills condition $($ TCCP $)$. From the fact that $f_{1}\left(t, x, 0, \zeta^{\prime}\right)=f_{2}(t, x, \zeta, 0)=0$ we see that $\underline{f}_{1}=\underline{f}_{2}=0$ and $\underline{\rho}_{1}=\underline{\rho}_{2}=0, \underline{y}_{1}=\underline{y}_{2}=0$ are suitable. Take $\bar{\rho}_{1}>0$ arbitrary and $\bar{f}_{1}=0, \bar{y}_{1}=\bar{\rho}_{1}$. We have

$$
f_{1}\left(t, x, \bar{y}_{1}(t), \zeta^{\prime}\right) \leq 0=\bar{f}_{1}\left(t, \bar{y}_{1}(t)\right)
$$

for all $\zeta^{\prime} \geq 0$. Finally, from inequality

$$
\begin{aligned}
f_{2}\left(t, x, \zeta, \zeta^{\prime}\right) & =\alpha_{2}(t, x) \zeta^{p} \exp \left(\gamma-\frac{\gamma}{\zeta^{\prime}}\right) \\
& \leq \bar{\alpha}_{2} \bar{\rho}_{1}^{p} \exp (\gamma)
\end{aligned}
$$


fulfilled for all $\zeta^{\prime}>0$ and all $\zeta \in\left[0, \bar{y}_{1}(T)=\bar{\rho}_{1}\right]$, the constant function $\bar{f}_{2}\left(t, \zeta^{\prime}\right)=\bar{\alpha}_{2} \bar{\rho}_{1}^{p} \exp (\gamma)$, and the affine function $\bar{y}_{2}(t)=\bar{\alpha}_{2} \bar{\rho}_{1}^{p} t \exp (\gamma)+\bar{\rho}_{2}$, with $\bar{\rho}_{2}>0$ arbitrary, are suitable to complete condition $(T C C P)$.

A.5. Proof of Proposition 2.5. The pair $\left(f_{1}, f_{2}\right)$ satisfies the structure condition of TCCP-structured reaction functions with $l=3$ : indeed, take

$$
\begin{aligned}
& r_{1}(t, x)=\left(\alpha_{1}(t, x),-\alpha_{1}(t, x) a(t, x),-b(t, x)\right), \\
& h_{1}\left(\zeta^{\prime}\right)=\left(1,1, \zeta^{\prime}\right), \\
& g_{1}(\zeta)=\left(\zeta^{2}(1-\zeta), \zeta(1-\zeta), 1\right) ;
\end{aligned}
$$

and

$$
\begin{aligned}
& r_{2}(t, x)=\left(\alpha_{2}(t, x),-c(t, x), 0\right), \\
& h_{2}(\zeta)=(\zeta, 1,0), \\
& g_{2}\left(\zeta^{\prime}\right)=\left(1, \zeta^{\prime}, 0\right) .
\end{aligned}
$$

Let us show that $\left(f_{1}, f_{2}\right)$ fulfills condition $(T C C P)$ with $\underline{\rho}_{i} \bar{\rho}_{i}$, and $\underline{f}_{i}, \bar{f}_{i}$ given by 2.4). We take $\underline{y}_{i}=\underline{\rho}_{i}$, $\bar{y}_{i}=\bar{\rho}_{i}$ and $\underline{\rho}_{i}$ and $\bar{\rho}_{i}$ must satisfy

$$
\begin{gathered}
f_{1}\left(t, x, \underline{\rho}_{1}, \zeta^{\prime}\right) \geq 0, \forall \zeta^{\prime}, \underline{\rho}_{2} \leq \zeta^{\prime} \leq \bar{\rho}_{2}, \\
f_{1}\left(t, x, \bar{\rho}_{1}, \zeta^{\prime}\right) \leq 0, \forall \zeta^{\prime}, \underline{\rho}_{2} \leq \zeta^{\prime} \leq \bar{\rho}_{2}, \\
f_{2}\left(t, x, \zeta, \underline{\rho}_{2}\right) \geq 0, \forall \zeta, \underline{\rho}_{1} \leq \zeta \leq \bar{\rho}_{1}, \\
f_{2}\left(t, x, \zeta, \bar{\rho}_{2}\right) \leq 0, \forall \zeta, \underline{\rho}_{1} \leq \zeta \leq \bar{\rho}_{1} .
\end{gathered}
$$

We look for $\underline{\rho}_{i} \leq 0$ for $i=1,2, \bar{\rho}_{1} \geq 1$, and $\bar{\rho}_{2}>0$. For all $\zeta \in\left[\underline{\rho}_{1}, \bar{\rho}_{1}\right]$ we have

then, for obtaining (A.11), it suffices to set

$$
\begin{aligned}
f_{2}\left(t, x, \zeta, \underline{\rho}_{2}\right) & =\alpha_{2} \zeta-c \underline{\rho}_{2} \\
& \geq \bar{\alpha}_{2} \underline{\rho}_{1}-\underline{\rho}_{2},
\end{aligned}
$$

$$
\underline{\rho}_{2}=\frac{\bar{\alpha}_{2}}{\underline{c}} \underline{\rho}_{1}
$$

Similarly, for all $\zeta \in\left[\underline{\rho}_{1}, \bar{\rho}_{1}\right]$ we have

$$
\begin{aligned}
f_{2}\left(t, x, \zeta, \bar{\rho}_{2}\right) & =\alpha_{2} \zeta-c \bar{\rho}_{2} \\
& \leq \bar{\alpha}_{2} \bar{\rho}_{1}-\underline{c} \bar{\rho}_{2},
\end{aligned}
$$

so that, for obtaining (A.12), it suffices to set

$$
\bar{\rho}_{2}=\frac{\bar{\alpha}_{2}}{\underline{c}} \bar{\rho}_{1} .
$$

On the other hand, for all $\zeta^{\prime} \in\left[\underline{\rho}_{2}, \bar{\rho}_{2}\right]=\left[\frac{\bar{\alpha}_{2}}{\underline{c}} \underline{\rho}_{1}, \frac{\bar{\alpha}_{2}}{c} \bar{\rho}_{1}\right]$, we have (recall that $\bar{\rho}_{1} \geq 1>a$ )

$$
\begin{aligned}
f_{1}\left(t, x, \bar{\rho}_{1}, \zeta^{\prime}\right) & =\alpha_{1} \bar{\rho}_{1}\left(\bar{\rho}_{1}-a\right)\left(1-\bar{\rho}_{1}\right)-b \zeta^{\prime} \\
& \leq \underline{\alpha}_{1} \bar{\rho}_{1}\left(\bar{\rho}_{1}-a\right)\left(1-\bar{\rho}_{1}\right)-\underline{b} \frac{\bar{\alpha}}{\underline{c}} \underline{\rho}_{1}
\end{aligned}
$$

so that, for obtaining (A.10), it suffices to take $\underline{\rho}_{1}$ and $\bar{\rho}_{1}$ satisfying

$$
\bar{\rho}_{1}\left(\bar{\rho}_{1}-a\right)\left(1-\bar{\rho}_{1}\right) \leq \underline{\gamma} \underline{\rho}_{1} .
$$

By a similar calculation, for obtaining $\overline{A .9}$, it suffices to take $\underline{\rho}_{1}$ and $\bar{\rho}_{1}$ satisfying

$$
\underline{\rho}_{1}\left(\underline{\rho}_{1}-a\right)\left(1-\underline{\rho}_{1}\right) \geq \bar{\gamma} \bar{\rho}_{1} .
$$

Set $\underline{\rho}_{1}=-\underline{\gamma} \bar{\rho}_{1}$. Then (A.13) and A.14 yield

$$
\left\{\begin{array}{l}
\left(\bar{\rho}_{1}-a\right)\left(\bar{\rho}_{1}-1\right) \geq \underline{\gamma}^{2} \\
\left(\underline{\rho}_{1}-a\right)\left(\underline{\rho}_{1}-1\right) \geq \frac{\bar{\gamma}}{\underline{\gamma}} .
\end{array}\right.
$$


The first inequality is fulfilled for $\bar{\rho}_{1} \geq \underline{\gamma}+1$, the second for $\underline{\rho}_{1} \leq a-\sqrt{\frac{\bar{\gamma}}{\gamma}}$, i.e. for $\bar{\rho}_{1} \geq \frac{\sqrt{\frac{\bar{\gamma}}{\gamma}}-\underline{a}}{\underline{\gamma}}$. Hence (A.9) and (A.10) are satisfied for $\bar{\rho}_{1}$ satisfying the first condition in (2.4).

\section{Appendix B. An estimate FOR the Right DERIVATIVE}

For a proof of the following Lemma, refer to [1, Lemma 3.3].

Lemma B.1. Let $X$ be a Hilbert space, $T>0, G \in W^{1,1}(0, T, X)$ and $\Phi: X \rightarrow \mathbb{R} \cup\{+\infty\}$ be a convex proper lower semicontinuous functional. Let u satisfy

$$
\left\{\begin{array}{l}
\frac{d u}{d t}(t)+\partial \Phi(u(t)) \ni G(t) \text { for a.e. } t \in(0, T), \\
u(0) \in \overline{\operatorname{dom}(\partial \Phi)} .
\end{array}\right.
$$

Then the right derivative of $u$ satisfies for all $t \in] 0, T]$ the following estimate

$$
\left\|\frac{d^{+} u}{d t}(t)\right\|_{X} \leq \frac{1}{t} \int_{0}^{t}\left\|\frac{d u}{d t}(s)\right\|_{X} d s+\int_{0}^{t}\left\|\frac{d G}{d t}(s)\right\|_{X} d s .
$$

\section{ApPEndix C. BASIC NOTIONS ON VARIATIONAL CONVERGENCES}

Let $(\mathbb{T}, \tau)$ be a topological space, $\left(F_{n}, F\right)_{n \in \mathbb{N}}$ a sequence of functionals mapping $\mathbb{T}$ into $\mathbb{R} \cup\{+\infty\}$. The following notion of convergence, equivalent to the convergence of the epigraph of $F_{n}$ to the epigraph of $F$ in the Kuratowski-Painlevé sense, is of central importance in Calculus of Variations and Homogenization theory.

Definition C.1. The sequence $\left(F_{n}\right)_{n \in \mathbb{N}}$ (sequentially) $\Gamma$-converges to $\mathrm{F}$ at $\mathrm{x}$ in $\mathbb{T}$ iff both assertions hold:

(i) there exists a sequence $\left(x_{n}\right)_{n \in \mathbb{N}}$ of $\mathbb{T}$, converging to $\mathrm{x}$, such that

$$
F(x) \geq \limsup _{n \rightarrow+\infty} F_{n}\left(x_{n}\right),
$$

(ii) for every sequence $\left(y_{n}\right)_{n \in \mathbb{N}}$, converging to $\mathrm{x}$ in $\mathbb{T}$,

$$
F(x) \leq \liminf _{n \rightarrow+\infty} F_{n}\left(y_{n}\right) .
$$

When (i) and (ii) hold for every $x$ in $\mathbb{T}$, we say that $\left(F_{n}\right)_{n} \Gamma$-converges to $\mathrm{F}$ in $(\mathbb{T}, \tau)$ and we write $F=\Gamma-\lim F_{n}$.

The main interest of this concept is its variational nature made precise in the first item of the following proposition.

Proposition C.1. Assume that $\left(F_{n}\right)_{n} \Gamma$-converges to $F$.

(i) Let $x_{n} \in \mathbb{T}$ be such that $F_{n}\left(x_{n}\right) \leq \inf \left\{F_{n}(x): x \in \mathbb{T}\right\}+\varepsilon_{n}$, where $\varepsilon_{n}>0$, $\varepsilon_{n} \rightarrow 0$. Assume furthermore that $\left\{x_{n}, n \in \mathbb{N}\right\}$ is $\tau$-relatively compact, then any cluster point $\bar{x}$ of $\left\{x_{n}, n \in \mathbb{N}\right\}$ is a minimizer of $F$ and

$$
\lim _{n \rightarrow+\infty} \inf \left\{F_{n}(x): x \in \mathbb{T}\right\}=F(\bar{x}) .
$$

(ii) If $G: X \rightarrow \mathbb{R}$ is continuous, then $\left(F_{n}+G\right)_{n} \Gamma$ to $F+G$.

For a proof and more about $\Gamma$-convergence, we refer the reader to Attouch [3] and Dal Maso [8]. We now consider the case where $(\mathbb{T}, \tau)$ is a Banach space $(V,\|\|$.$) . Being endowed with strong and weak$ topology, we have two notions of $\Gamma$-convergence. Given a sequence $\left(\Phi_{n}\right)_{n \in \mathbb{N}}$ of functionals $\Phi_{n}: V \rightarrow$ $\mathbb{R} \cup\{+\infty\}$, according to Definition C.1, we denote by $\Gamma_{w}-\lim \Phi_{n}$ and $\Gamma_{s}-\lim \Phi_{n}$ the $\Gamma$-limits associated with the weak and the strong convergence in $V$ respectively, when they exist.

Definition C.2 (Mosco convergence). Let $(V,\|\|$.$) be a Banach space, a sequence \left(\Phi_{n}\right)_{n \in \mathbb{N}}$ of extended real-valued functions $\Phi_{n}: V \rightarrow \mathbb{R} \cup\{+\infty\}$, and $\Phi: V \rightarrow \mathbb{R} \cup\{+\infty\}$. The sequence $\left(\Phi_{n}\right)_{n \in \mathbb{N}}$ Moscoconverges to $\Phi$ and we write $\Phi_{n} \stackrel{M}{\rightarrow} \Phi$ if

$$
\Phi=\Gamma_{w}-\Phi_{n}=\Gamma_{s}-\Phi_{n} .
$$


The argument which naturally led us to introduce the Mosco-convergence notion yields the bicontinuity of the Fenchel duality transformation in the context of convex functions. More precisely

Theorem C.1. Let $(V,\|\|$.$) be a reflexive Banach space and \left(\Phi_{n}\right)_{n \in \mathbb{N}}$, $\Phi$ a sequence of convex proper lower semicontinuous functions from $V$ into $\mathbb{R} \cup\{+\infty\}$. The following statements are equivalent:

(i) $\Phi_{n} \stackrel{M}{\rightarrow} \Phi$ on $V$;

(ii) $\Phi_{n}^{*} \stackrel{M}{\rightarrow} \Phi^{*}$ on $V^{*}$.

For a proof, we refer the reader to [4, Theorem 17.4.3].

The following Proposition whose proof is straightforward, states an equivalent formulation interesting from practical point of view.

Proposition C.2. Let $(V,\|\|$.$) be a reflexive Banach space, and \left(\Phi_{n}\right)_{n \in \mathbb{N}}, \Phi$ a sequence of convex proper lower semicontinuous functions from $V$ into $\mathbb{R} \cup\{+\infty\}$. The following statements are equivalent:

(i) $\Phi_{n} \stackrel{M}{\rightarrow} \Phi$;

(ii) $\forall v \in V, \exists v_{n} \rightarrow v$ such that $\Phi_{n}\left(v_{n}\right) \rightarrow \Phi(v)$;

$\forall v \in V, \forall v_{n} \rightarrow v, \Phi(v) \leq \liminf _{n \rightarrow+\infty} \Phi_{n}\left(v_{n}\right) ;$

(iii) $\forall v \in V, \exists v_{n} \rightarrow v$ such that $\Phi_{n}\left(v_{n}\right) \rightarrow \Phi(v)$, $\forall v^{*} \in V^{*}, \exists v_{n}^{*} \rightarrow v^{*}$ such that $\Phi_{n}^{*}\left(v_{n}\right) \rightarrow \Phi^{*}(v)$.

\section{REFERENCES}

[1] O. Anza Hafsa, J.-P. Mandallena, G. Michaille. Stability of a class of nonlinear reaction-diffusion equations and stochastic homogenization. To appear in Asymptotic Analysis.

[2] O. Anza Hafsa, J.-P. Mandallena, G. Michaille. Groupe de recherche Mathématiques en Cévennes. Convergence of a class of nonlinear reaction-diffusion equations and stochastic homogenization http: // mipa. unimes. fr/ preprints/ MIPA-Preprint03-2016. pdf.

[3] H. Attouch. Variational convergence for functions and operators. Applicable Mathematics Series. Pitman (Advanced Publishing Program), Boston, MA, 1984.

[4] H. Attouch, G. Buttazzo, G. Michaille. Variational analysis in Sobolev and BV space: application to PDEs and Optimization, Second Edition. MPS-SIAM Book Series on Optimization 6, Oct 12014.

[5] H. Brézis. Opérateurs maximaux monotones et semi-groupes de contractions dans les espaces de Hilbert. North-Holland mathematics studies 5, 1973.

[6] G. Dal Maso, L. Modica. Nonlinear stochastic homogenization and ergodic theory. J. Reine Angew. Math. 363 (1986), $27-43$.

[7] C. Collins. Length dependence of solutions of FitzHugh-Nagumo equations. Trans. Amer. Math. Soc. Vol 280, 2 (1983), 809-832.

[8] Gianni Dal Maso. An introduction to $\Gamma$-convergence. Progress in Nonlinear Differential Equations and their Applications, 8. Birkhäuser Boston Inc., Boston, MA, 1993.

[9] A. Dikansky. FitzHugh-Nagumo equations in a nonhomogeneous medium. Disc. Cont. Dyn. Sys. Supplement Volume (200), 216-224.

[10] A. Mielke, S. Reichelt, M. Thomas. Two-scale homogenization of nonlinear reaction-diffusion systems with slow diffusion. Netw. Heterog. Media 9(2), ( 2014) 353382.

[11] J.D. Murray. Mathematical Biology: I. An Introduction, Third Edition. Interdisciplinary Applied Mathematics, Volume 17, Springer-Verlag New York Berlin Heidelberg, 2002.

[12] C.V. Pao. Nonlinear parabolic and elliptic equations. Plenum Press, New York, 1992.

[13] M. A. Peter. Coupled reaction-diffusion processes inducing an evolution of the microstructure: analysis and homogenization. Nonlinear Anal. 70(2), (2009) 806821.

[14] S. Reichelt. Two-Scale Homogenization of Systems of Nonlinear Parabolic Equations DISSERTATION, zur Erlangung des akademischen Grades doctor rerum naturalium (Dr. rer. nat.) eingereicht an der MathematischNaturwissenschaftlichen Fakultät der Humboldt-Universität zu Berlin, 2015.

[15] M.E. Shonbek. Boundary Value Problems for the FitzHugh-Nagumo equations. J. Differ. Equat. 30 (1978), 119-147. 